\title{
A Decision-Analysis Framework for Comparing Experimental Designs \\ of Projects to Enhance Pacific Salmon
}

by

\author{
Brice MacGregor \\ B.A., University of British Columbia, 1995 \\ M.A., Queen's University, 1996 \\ RESEARCH PROJECT SUBMITTED IN PARTLAL FULFLLMENT OF \\ THE REQUIREMENTS FOR THE DEGREE OF \\ MASTER OF RESOURCE MANAGEMENT \\ in the School \\ of
}

Resource and Environmental Management

Project No. 257

C Brice MacGregor 2000

SIMON FRASER UNIVERSITY

May 2000

All rights reserved. This work may not be reproduced in whole or in part, by photocopy or any other means, without permission of the author. 
National Library of Canada

Acquisitions and Bibliographic Services

395 Wellington Street Onawa ON KIA ONM Cenada
Btbliotheque nationale du Canada

Acquisitions et senvices bibliographiques

395, nue Wollington Otswa ON KIA ONA Cenada
The author has granted a nonexclusive licence allowing the National Library of Canada to reproduce, loan, distribute or sell copies of this thesis in microform, paper or electronic formats.

The author retains ownership of the copyright in this thesis. Neither the thesis nor substantial extracts from it may be printed or otherwise reproduced without the author's permission.
L'auteur a accordé une licence non exclusive permettant à la Bibliothèque nationale du Canada de reproduire, prêter, distribuer ou vendre des copies de cette thèse sous la forme de microfiche/film, de reproduction sur papier ou sur format électronique.

L'auteur conserve la propriété du droit d'auteur qui protège cette thèse. $\mathrm{Ni}$ la thèse ni des extraits substantiels de celle-ci ne doivent être imprimés ou autrement reproduits sans son autorisation.

\section{Canadầ}




\begin{abstract}
Wise investment in fisheries enhancement projects for Pacific salmon requires rigorous evaluation of their performance through monitoring. Monitoring a number of enhanced and non-enhanced (control) stocks may generate information useful to future investment decisions in such projects. However, the costs of monitoring can be quite substantial. To address this tradeoff, I developed a decision analysis framework for choosing among monitoring programs for experimental management strategies for enhancement projects. This framework explicitly evaluates different options in terms of the value of information generated and costs of obtaining that information through monitoring. I then applied this framework to groundwater-fed side channels utilized by coho and chum salmon. Generally, taking an experimental approach to enhancement (i.e. monitoring prior to making an investment decision) yielded positive expected net benefits when compared to the option of enhancing all relevant systems without first monitoring. Optimal monitoring programs were small (i.e. few systems and years monitored) owing to the large costs of monitoring, implying that in cases similar to the one studied here, the large costs of adopting monitoring programs with high statistical power may not outweigh the benefits.
\end{abstract}




\section{ACKNOWLEDGEMENTS}

For his advice and encouragement, I thank my senior supervisor Randall

Peterman. I also thank Brian Pyper for many conceptual discussions and additional insights that helped generate this analysis. I also acknowledge Michael Bradford for discussing analyses and reviewing several drafts of this manuscript. I also thank Greg Steer and Greg Bonnell for valuable discussions on various aspects of my work. Finally, I would like to thank Jim Irvine and Chris Sporer who provided some of the data that went into this analysis. This project was funded by the Canadian Department of Fisheries and Oceans, a NSERC Grant to Randall Peterman, and a graduate fellowship from Simon Fraser University. 


\section{TABLE OF CONTENTS}

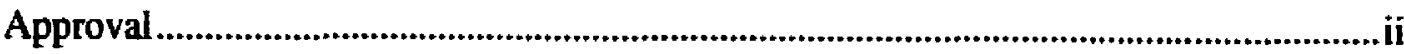

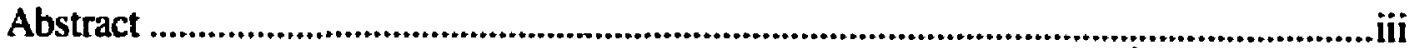

Acknowledgments ............................................................................................................ iv

List of Tables ................................................................................................................. vi

List of Figures.................................................................................................................viii

I. $\quad$ NTRODUCTION

II. BIOLOGICAL MODEL FOR THE EFFECTS OF GROUNDWATER-FED SIDE CHANNELS ON COHO SALMON......................................................................

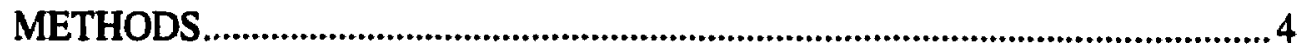

RESULTS AND DISCUSSION................................................................... 21

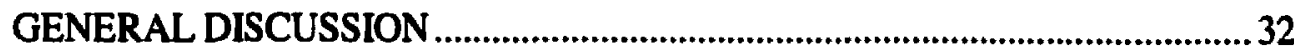

III. DECISION ANALYSIS FOR DETERMINING OPTIMAL EXPERIMENTAL DESIGN FOR PROGRAMS TO MONITOR GROUNDWATER-FED SIDE

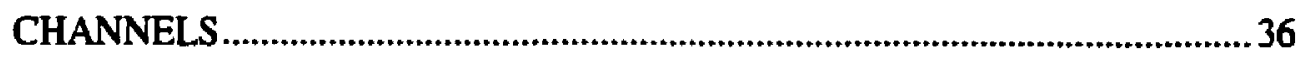

INTRODUCTION

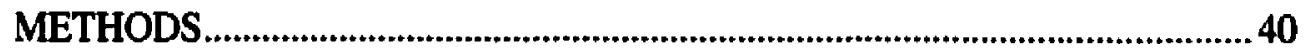

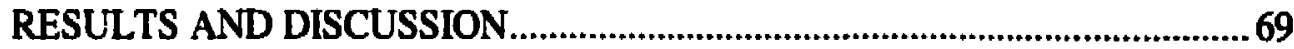

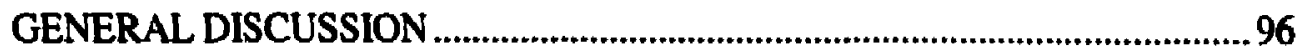

IV. SUMMARY AND CONCLUSIONS

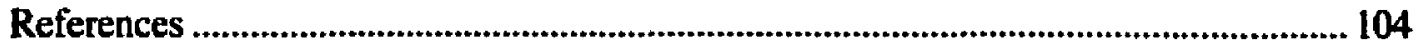

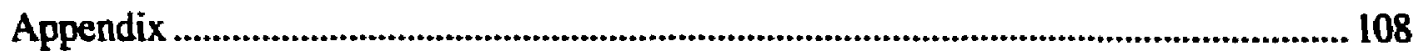




\section{LIST OF TABLES}

Table

1 Baseline parameter values used in the biological simulation model and calculation of the net present value of groundwater-fed side channels

2 Summary statistics for baseline simulations of the biological model under four scenarios with respect to the present/absence of a groundwater-fed side channel and "incubation" (egg-to-fry survival rate) and "rearing" effects (fry-to-smolt survival rate).

3 Average annual smolt production over first 5 generations for various combinations of incubation and rearing effects assuming the groundwater-fed side channel comprises 16.7 percent of total spawning habitat. $\theta_{1}$ and $\theta_{2}$ refer to the effects of enhancement on coho egg-to-fry and fry-to-smolt survival rates, respectively, as defined by equations (6) and (II)

4 Generation in which hypothetical coho population is first fully seeded assuming the groundwater-fed side channel comprises 16.7 percent of total spawning habitat. $\theta_{1}$ and $\theta_{2}$ refer to the effects of enhancement on coho egg-to-fry and fryto-smolt survival rates, respectively, as defined by equations (6) and (II)

5 Average annual smolt production over first 5 generations for various combinations of incubation and rearing effects assuming the groundwater-fed side channel comprises 28.6 percent of total spawning habitat. $\theta_{1}$ and $\theta_{2}$ refer to the effects of enhancement on coho egg-to-fry and fry-to-smolt survival rates, respectively, as defined by equations (6) and (11)

6 Generation in which hypothetical coho population is first fully seeded assuming the groundwater-fed side channel comprises 28.6 percent of total spawning habitat. $\theta_{i}$ and $\theta_{2}$ refer to the effects of enhancement on coho egg-to-fry and fryto-smolt survival rates, respectively, as defined by equations (6) and (11)

7 Methods of sampling and assumed coefficients of variation $(\sigma / \mu)$ in abundance estimates for high-precision monitoring program. See Appendix for derivation of coefficients of variation

8 Methods of sampling and assumed coefficients of variation $(\sigma / \mu)$ in abundance estimates for low-precision monitoring program. See Appendix for derivation of coefficients of variation

9 Measurement error variance for effect sizes with high-precision sampling methods 
10 Measurement error variance for effect sizes with low-precision sampling methods

11 Baseline parameter values for decision analysis on alternative monitoring programs for groundwater-fed side channels

12 Summary of parameter values for sensitivity analyses performed in decision analysis on alternative monitoring programs for groundwater-fed side channels. Parameter values and assumptions in each case are the same as the baseline case except where stated otherwise

13 Summary of performance measures for decision analysis with baseline parameter values 77

14 Summary of performance measures for decision analysis with different prior probability distributions for the proportional increase in the chum egg-to-fry survival rate $\left(\exp \left(\mu_{3}\right)-1\right)$

15 Summary of performance measures for decision analysis with different real discount rates 


\section{LIST OF FIGURES}

Figure

I Flow chart of the life-history stages of coho salmon considered in simulations of the effects of enhanced side-channel habitat on abundance of salmon and rates of stock-rebuilding.

$2 \quad$ Hypothetical relationships between total smolts produced and presmolt abundances for presmolts rearing in enhanced side channels and in wild habitat. $\theta_{2}$ denotes the "rearing effect" or incremental increase in smolt production experienced by presmolts rearing in enhanced side channels

3 Hypothetical relationships between presmolt-to-smolt survival rates and presmolt abundances for presmolts rearing in enhanced side channels and in wild habitat. $\theta_{2}$ denotes the "rearing effect" or incremental increase in smolt production experienced by presmolts rearing in enhanced side channels

4 Simulated smolt production over 10 generations for four model scenarios. See text for details.

5 Smolt outmigration and incremental smolt production for a fully-seeded system ( 180 female spawners) and a system that is not fully-seeded ( 90 female spawners) for various levels of "incubation effects" $\left(\theta_{l}\right)$, "rearing effects" $\left(\theta_{2}\right)$, and rearing preferences $(\beta)$

6 Smolts produced by the system with and without side channels as a function of female spawner abundance for baseline parameter values

7 Smolt outmigration and incremental smolt production as a function of female spawner abundance for baseline parameter values.

8 A hypothetical linear relationship between the net present value of an enhancement project as a function of the magnitude of the biological effect of enhancement

9 Decision tree for comparing monitoring programs. See text for details

10 Flow chart for the decision analysis model used to compare alternative monitoring programs for groundwater-fed side channels

11 Timeline indicating the sequence of events and the timing of costs and benefits in a hypothetical monitoring program for groundwater-fed side channels. 
12 Net present value of a side channel as a function of the proportional increases in the: (A) coho egg-to-fry survival rate, (B) coho fry-to-smolt survival rate, and (C) chum egg-to-fry survival rate

13 Net present value of a side channel as a function of the coho marine survival rate

14 Net present value of a side channel as a function of the real discount rate .72

15 The probability that monitoring programs of different duration ( 1 or 2 years) and different numbers of systems (I to 10 systems) lead to a correct decision regarding whether to apply enhancement more widely with the baseline 73

16 The expected economic benefit of decisions regarding whether to apply enhancement further for various monitoring programs with baseline parameter assumptions

17 The expected net economic benefit of various monitoring programs with baseline parameter assumptions .75

18 Frequency distributions for the number of coho recruits in year 7 of the postexperimental period with: (A) the "economically optimal" monitoring program, (B) "perfect information", and (C) "enhancement without monitoring"

19 The expected net economic benefit of "economically optimal" monitoring programs for all the scenarios investigated in the decision analysis

20 Expected net economic benefit of monitoring programs with the "high-precision" and "low precision" sampling methods with optimistic and pessimistic assumptions for measurement error variance.

21 Expected net economic benefit of monitoring programs with high and low process error variance.

22 Expected net economic benefit of monitoring programs for various assumptions regarding the prior probability distribution for the true mean proportional increase in the chum egg-to-fry survival rate $\left(\exp \left(\mu_{3}\right)-1\right)$

23 Expected net economic benefit of monitoring programs for various assumptions regarding the total number of non-experimental systems to which the results of the monitoring program can be applied.

24 Expected net economic benefit of monitoring programs with different real discount rates 
25 Expected net economic benefit of monitoring programs for various assumptions regarding the standard deviation of system-specific effects.

26 Expected net economic benefit of monitoring programs with: (A) no year-specific effects, (B) moderate variability in year-specific effects, and (C) high variability in year-specific effects. .95 


\section{Introduction}

The Canada Department of Fisheries and Oceans (DFO) is involved in many enhancement projects for Pacific saimon (Oncorhynchus spp.). Wise investment in these projects requires rigorous evaluation of their performance. However, many biotic and abiotic factors simultaneously affect the survival rates of Pacific salmon at various stages in their life histories. This creates a challenge for evaluation because natural variability, measurement error, and other factors that change unintentionally may obscure the effects of enhancement on salmon populations. This may be particularly problematic for smallscale enhancement projects, in which the effects of enhancement may be small in relation to the inherent variability in survival rates of affected populations.

To distinguish between the effects of enhancement and other sources of variation, experimental design considerations should be incorporated into monitoring programs for enhancement projects. However, many designs are possible, so an important question is, which design is most appropriate? Given that costs of monitoring and evaluation can be substantial (e.g. from estimating abundances of eggs, fry, and smolts of salmon each year), there are limitations to the number of systems and years that can be monitored before deciding whether to apply a particular method of enhancement more widely. Is it best to monitor several systems for a short period or a few systems for longer? Furthermore, some methods of estimating abundances may be more reliable than others (e.g. counting fences), but if the more reliable methods cost more, is the better information that they generate worth the extra cost? Obviously, the more years and systems that are monitored and the more precise the sampling methods that are used to estimate the biological effects of enhancement, the more likely managers will be to make 
correct investment decisions in the future, hence avoiding potentially large costs when enhancement does not work well, and enjoying large gains in salmon production when it does.

To answer these and related questions, I developed a quantitative framework based on decision analysis, which is an analytical tool designed to help make decisions in the presence of uncertainty (Clemen 1996; Peterman and Anderson 1999). This framework explicitly evaluates different monitoring programs in terms of their costs and the value of information obtained. I applied this framework to groundwater-fed side channels employed to enhance coho and chum salmon.

I present this analysis in two stages. In the first stage, I constructed a biological model to simulate the effects of groundwater-fed side channels on a hypothetical coho stock. Specifically, the model simulates the dynamics of a coho population over successive generations given life-stage-specific and habitat-specific survival rates. The main purpose of this model was to link coho population dynamics to measurable biological effects of enhancement, where the biological effects are the incremental increases in (1) the egg-to-fry survival rate for coho eggs incubating in side channels and (2) the fry-to-smolt survival rate for coho fry rearing in the side channel. Clearly, the magnitude of these effects has important implications for the efficacy of using side channels to enhance coho salmon. However, due to the uncertainty in the magnitude of these effects, there may be benefits to monitoring existing projects before deciding to invest in building more side channels.

In the second stage of my analysis, I used the biclogical model developed in the first stage in a formal decision analysis to determine the appropriate experimental design 
(i.e. the experimental design yielding the greatest expected net economic benefits) for monitoring programs used to quantify the biological effects of side channels. Using an approach analogous to that of Walters and Green (1997), I evaluated the tradeoffs between the value of additional information (reducing our uncertainty regarding effects of enhancement) versus the costs of obtaining additional information (costs of monitoring the effects of side channels). 


\section{Biological model for the effects of groundwater-fed side channels on coho salmon}

\section{METHODS}

\section{Model Overview}

The biological model in this first-stage analysis deterministically simulates the dynamics of a coho stock over successive generations. Recruitment of spawners from one generation to the next is determined by fecundity and life-stage-specific and habitatspecific survival rates. In the model, there are two types of habitat: (1) side channels (enhanced) and (2) natural or wild. The proportions of juvenile and spawning fish partitioned into enhanced and wild habitat are based on the relative areas of enhanced and wild habitat for the appropriate life stages. A flow chart for the biological model (not the entire decision-analysis model) is shown in Figure $\mathrm{I}$. More details on each stage of the biological model are described below.

\section{Model Details}

Spawners and Spawner Allocation

Spawners for the first generation are an input to the model. For successive generations, spawners are determined by the following equation:

(1) Spawners $=$ Smolts $_{\text {tot }} \bullet$ Marine

where Smolts ${ }_{t a t}$ is the total number of smolts produced by the stock and Marine is the marine survival rate. 
Figure 1. Flow chart of the life-history stages of coho salmon considered in simulations of the effects of enhanced side-channel habitat on abundance of salmon and rates of stock-rebuilding.

Initial Spawners

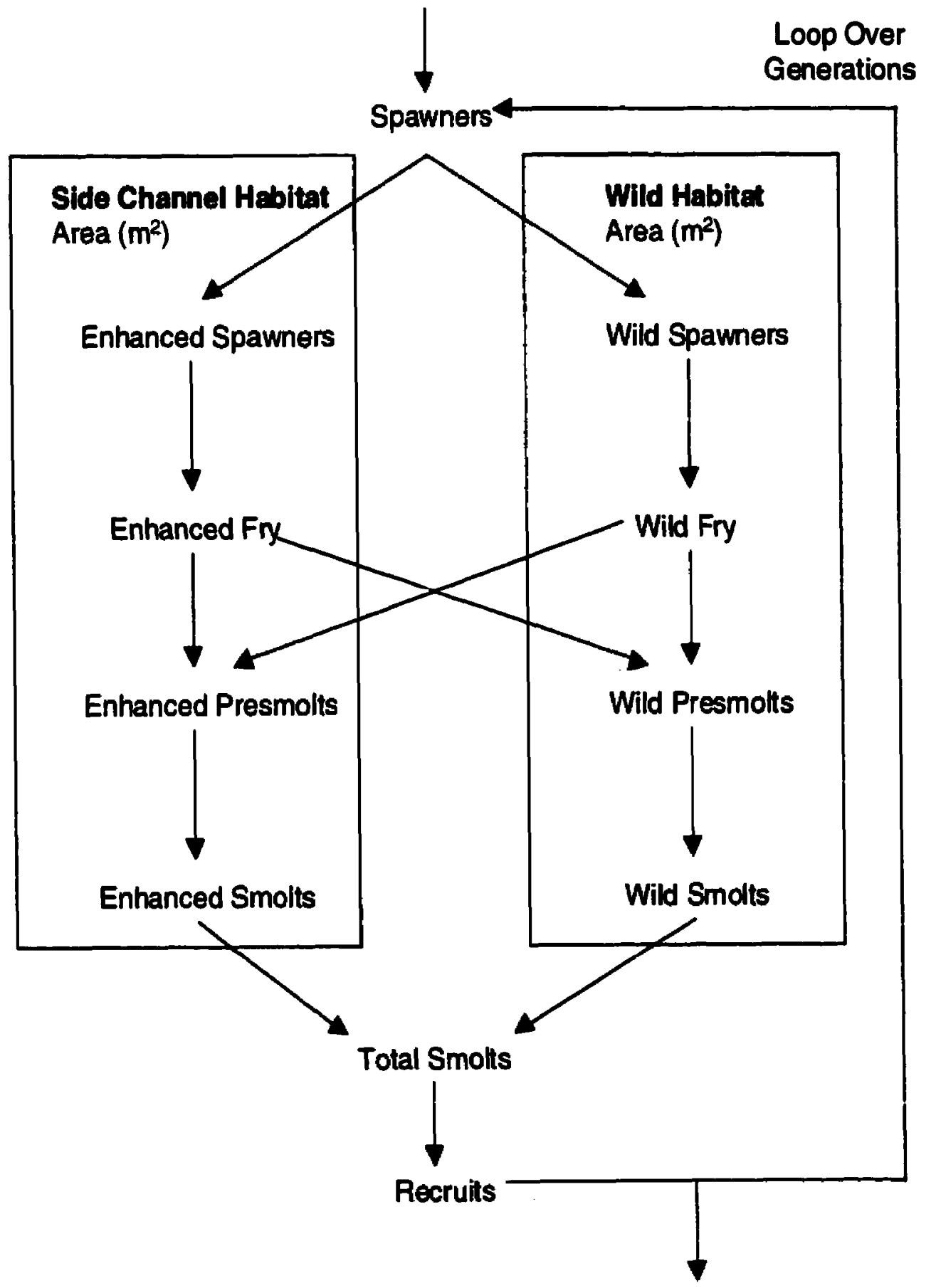

Calculate number of generations until fully seeded 
The number of female spawners is the number of spawners times the proportion of females $(P f)$. A 1:1 sex ratio has been assumed. Spawning females are allocated into enhanced $(E)$ and wild $(W)$ habitat using the following two equations:

$$
\text { Females }_{\varepsilon}=\text { Females } \bullet\left(\frac{\alpha \bullet \text { SideArea }}{\alpha \bullet \text { SideArea }+ \text { SpawnArea }}\right)
$$

(3) $\quad$ Females $_{\mathrm{w}}=$ Females - Females $\mathrm{E}_{E}$

where SideArea is the total area of the side channel, SpawnArea is the total area of natural spawning habitat and $\alpha$ is a preference parameter. Where $\alpha=1$, female spawners show no preference for side channel habitat over natural spawning habitat, and the allocation of fernales to each habitat type is proportional to their total areas. Where $\alpha>1$, a disproportionately large number of spawners will use side channels, while the opposite is true if $\alpha<1$.

There are studies that show fry and/or smolt production of side channels by number of spawners and spawner density for chum (Bonnell, 1991; Cowan, 1991) and coho (Sheng et al. 1990). However, none of these studies provide data on spawner density in adjacent wild spawning habitat. Hence, it is not possible to determine $\alpha$ from the literature (i.e. the extent to which spawners may, or may not, prefer side channels to wild spawning habitat). I assumed $\alpha=1$ for preliminary model runs.

Fecundity and Egg Deposition

The number of eggs deposited in each habitat type is determined by the following equations:

(4) $E g g s_{E}=$ Females $_{E} \bullet$ Fecundity 


$$
\text { Eggs }_{W}=\text { Females }_{W} \bullet \text { Fecundity }
$$

where Fecundity is the number of eggs each female lays. Sandercock (1991) reviews fecundity of coho females from existing literature. Mean values for BC stocks lie between 2300 to 2700 eggs per female. Sheng et al. (1990) report an average fecundity of 2,495 eggs per female for coho in the Upper Paradise Creek channel in British Columbia (37 fish sampled from 1984-1986). Hence, a value of 2,500 has been assumed in the model.

\section{Fry Production}

Since fry abundance for coho is linearly related to spawner abundance (Bradford et al. 2000), given the current low abundances, I assumed that egg-to-fry survival rates in both wild and enhanced habitat are density-independent and constant. The equations determining fry production (i.e. annual abundance) from enhanced and wild areas are: (6) $\quad F r y_{E}=a \bullet \exp \left(\theta_{1}\right) \bullet E g g s_{E}$

(7) $\quad F r y_{W}=a \bullet E g g s_{W}$

where $a$ is the wild egg-to-fry survival rate and $\exp \left(\theta_{l}\right)$ is the multiplicative effect of enhancement on the egg-to-fry survival rate. $\theta_{l}$ determines the multiplicative increase in egg-to-fry survival for enhanced fish. Specifically,

$$
\theta_{l}=\ln \left(1+\text { PerIncrease }_{E F}\right)
$$

where Perlncrease ${ }_{E F}$ is the proportional increase in egg-to-fry survival rate (Fry/Egg) for enhanced fish above the wild survival rate. Total fry production is the sum of (6) and (7).

Sheng et al. (1990) provide estimates of egg-to-fry survival for coho by spawner density in 3 side channels in British Columbia based on visual counts of spawners and 
downstream trap counts of fry emerging from side channels $(\mathrm{N}=12)$. The average egg-tofry survival rate from these channels was $\mathbf{1 8 . 2}$ percent, compared to an average egg-to-fry survival rate for wild coho from other sources of $\mathbf{2 0}$ percent (reviewed in Bradford, 1995). This suggests that side-channels do not provide any incremental survival rate advantage to progeny of coho salmon spawning in them. Coho salmon have a higher average egg-to-fry survival rate than pink, chum, and sockeye salmon (Bradford, 1995), which may be the result of differences in site selection for spawning. Coho salmon generally spawn in areas with high-quality gravel in the headwaters of streams, which may be the reason for their higher egg-to-fry survival rates and the reason for the lack of a survival advantage for coho eggs incubating in side channels (Michael Bradford, pers. comm.). However, owing to the lack of experimental design upon which this comparison of survival rates in the literature is based, it is difficult to draw a firm conclusion.

Conversely, data for chum salmon indicate that there may be a significant survival advantage to chum eggs incubating in side channels. Bonnell (1991) provides estimates of egg-to-fry survival rates of chum salmon from a number of groundwater-fed side channels in British Columbia. He reports an average egg-to-fry survival rate of 16.3 percent $(\mathrm{N}=30)$ for chum eggs in these side channels. Cowan (1991) estimates egg-to-fry survival rate of chum eggs in 5 Washington State side channels of 35.6 percent $(\mathrm{N}=15)$. The large differences in survival rates may be attributable to differences in spawner density in the two studies; Bonnell (1991) reports an average of 0.8 females per $\mathrm{m}^{2}$ of side channel, while mean female density was only 0.15 in the channels studied by Cowan (1991). The egg-to-fry survival rates in these studies compare to average values from the literature for wild chum of approximately 9 percent (Bradford, 1995). 


\section{Allocation of Pre-smolts}

Fry produced by side channels may leave these channels and take up residence in other parts of the stream. Conversely, juveniles from other parts of the system may use side channels for a portion of their freshwater residence. To account for possible mixing between wild and enhanced fry, I partitioned the total number of fry into fish rearing in wild areas only and those using the side channel, based on the relative areas of those two habitat types. To avoid confusion with the fry production stage of the model, I refer to fry categorized by rearing habitat as pre-smolts. The following equations determine this division:

(9) $\quad$ PreSmolts $_{E}=$ Fry $_{t \mathrm{ut}} \bullet\left(\frac{\beta \bullet \text { SideArea }}{\beta \bullet \text { SideArea }+\lambda \bullet \text { WildArea }}\right)$

(10) $\quad$ PreSmolts $_{W}=F r y_{t m t}-$ PreSmolts $_{E}$

where SideArea is the total area of the side channel, WildArea is the total area of natural rearing habitat, $\beta$ is a preference parameter, and $\lambda$ is a scaling parameter that translates the area of wild habitat into an equivalent area of side channel habitat that could produce the same number of coho smolts.

There is ample evidence that off-channel habitats contribute a disproportionately large number of smolts to total outmigration when habitat types are compared on the basis of area (Brown and Hartman, 1988; Decker 1998). Bradford et al. (1997) provide estimates of coho smolt abundance for both side channels and wild habitat. However, these data are not sufficient to indicate the preference (if any) that coho juvenile might have for side channel habitat versus wild habitat, because differences in smolt production 
are only partially attributable to habitat preference. Other variables affecting smolt outmigration are differences in the amount of suitable rearing habitat per unit of area and differences in survival rates that coho experience in these different habitat types. Hence, I assumed $\beta=1$.

The scaling parameter $(\lambda)$ is the ratio of maximum smolt production per unit area in wild and enhanced habitat. Estimates of maximum production per unit area for each habitat type are discussed below.

\section{Smolt Survival and Production}

I used the "hockey stick" model (Bradford et al. 2000) (Figure 2) to simulate smolt production from each habitat type. Smolts produced in enhanced and wild areas are determined by the following equations:

(11) Smolts $_{E}=\min \left\{b \bullet \exp \left(\theta_{2}\right) \bullet\right.$ PreSmolts $_{E}$, MaxSmolts E $_{E} \bullet$ SideArea $\}$

(12) Smolts $_{W}=\min \left\{b \bullet\right.$ PreSmolts $_{W}$, MaxSmolts $_{W} \bullet$ WildArea $\}$

where $b$ is the density-independent fry-to-smolt survival rate for fish rearing only in wild areas, $\exp \left(\theta_{2}\right)$ is the multiplicative effect on the fry-to-smolt survival for juveniles rearing in side channels, and MaxSmolts $s_{E}$ and MaxSmoltsw are the maximum number of smolts that can be produced per unit of area $\left(\mathrm{m}^{2}\right)$ of side channel and wild habitat respectively. Total smolt production from the entire system is the sum of (11) and (12).

Smolt production and fry-to-smolt survival rate relationships are shown in Figures 2 and 3 respectively. In Figure 2, the ascending limb for the smolt production curve is steeper for the side channel habitat, reflecting benefits to juveniles rearing in side 
Figure 2. Hypothetical relationships between total smolts produced and presmolt. abundances for presmolts rearing in enhanced side channels and in wild habitat. $\theta_{2}$ denotes the "rearing effect" or incremental increase in smolt production experienced by presmolts rearing in enhanced side channels.

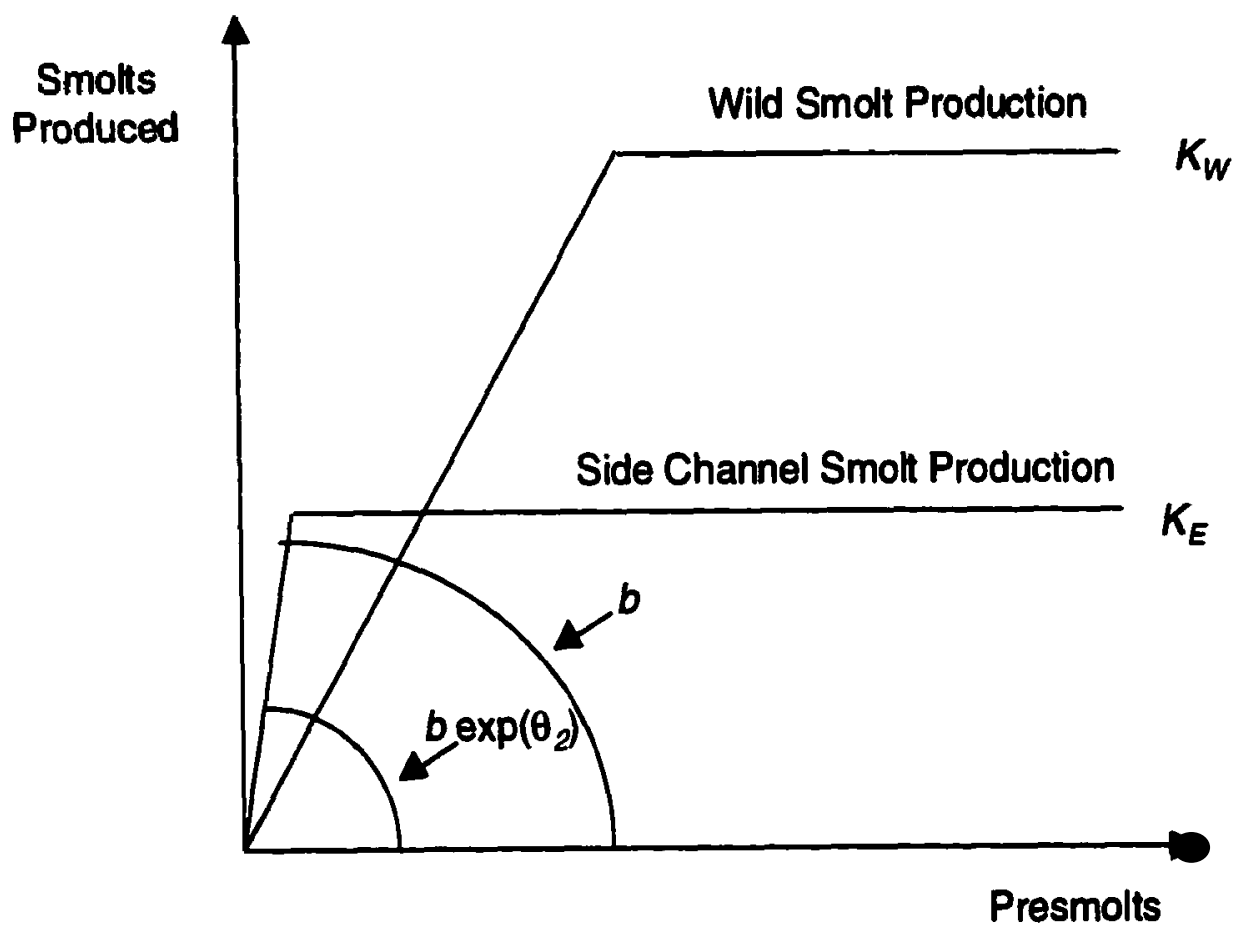


Figure 3. Hypothetical relationships between presmolt-to-smolt survival rates and presmolt abundances for presmolts rearing in enhanced side channels and in wild habitat. $\theta_{2}$ denotes the "rearing effect" or incremental increase in smolt production experienced by presmolts rearing in enhanced side channels.

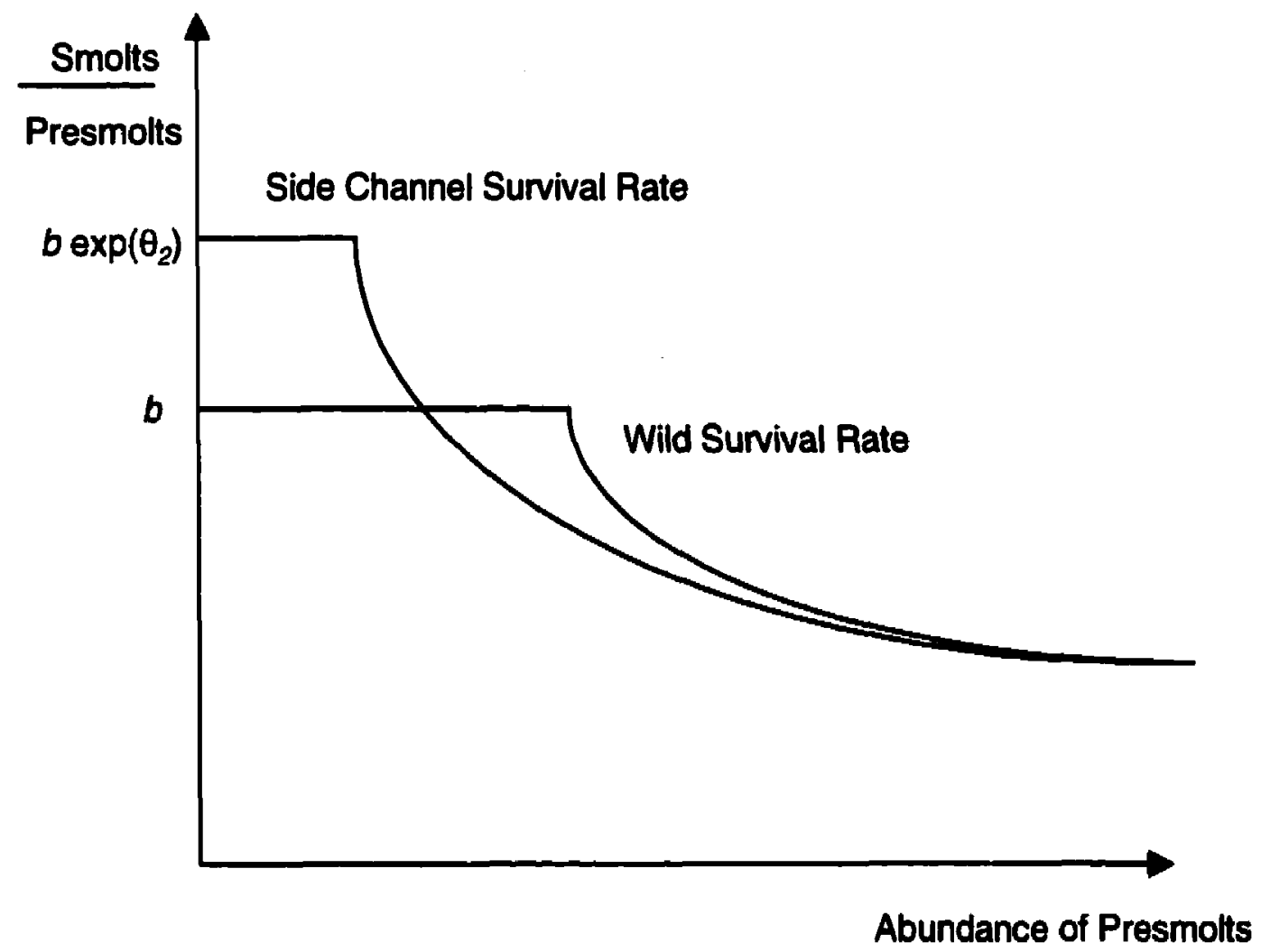


channels in terms of survival rate at low abundance. The ceilings for smolt production in wild and enhanced areas reflect the relative carrying capacities for each habitat type. This example shows the case where the side channel is only a small portion of the total rearing habitat. However, it may the case that a side channel represents a large proportion of total rearing habitat in systems without much natural side channel and off-channel habitat for rearing juveniles. Carrying capacities for side channels and wild habitat are denoted as $K_{E}$ and $K_{W}$ respectively. Figure 3 shows fry-to-smolt survival rate relationships for enhanced and wild habitat obtained by rearranging equations (11) and (12).

Data are available on fry-to-smolt survival rates for coho (reviewed by Bradford, 1995). However, these data may contain cases in which streams are fully seeded, as well as those which are not (i.e. where the carrying capacity of streams may or may not have been reached). In terms of equations ( 11 ) and (12), estimated survival rates from streams that are not fully seeded would provide an estimate of fry-to-smolt survival rates at low abundance (i.e. parameter $b$ in the model), while data from streams which are fully seeded will underestimate $b$. Hence, the average fry-to-smolt survival rate for coho of 7.4 percent reported by Bradford (1995), is likely to be a minimum estimate of the $b$ parameter.

However, data on coho smolt production by number of female spawners are available for some stocks. With these data, it is possible to determine the densityindependent number of smolts produced per female spawner. Using the following identity,

$$
\frac{\text { Smolts }}{\text { Female }}=\left(\frac{\text { Eggs }}{\text { Female }}\right) \cdot\left(\frac{\text { Fry }}{\text { Egg }}\right) \cdot\left(\frac{\text { Smolts }}{\text { Fry }}\right)
$$


we can substitute in values from the literature for average fecundity and egg-to-fry survival, leaving the fry-to-smolt survival rate (the last term above) as the only unknown in equation (13). Solving for this value yields a rough estimate for the $b$ parameter in the model.

Bradford et al. (1999) fit the "hockey stick" model to 14 coho stocks. They obtained an average estimate of 85 smolts per female at low spawner abundances. If we assume average fecundity of 2,500 eggs per female (Sandercock, 1991) and an average egg-to-fry survival of 20 percent (Bradford, 1995), this implies an average fry-to-smolt survival rate of 17 percent in cases where streams are not fully seeded.

Sufficient data were not available to determine the incremental increase in survival rate associated with coho juveniles rearing in side channels (i.e. $\theta_{2}$ ). In Carnation Creek, over-winter survival rates for juvenile coho in off-channel and mainchannel habitats were estimated to be 35 and 56 percent, respectively, in 1982-83, but were 43 and 34 percent, respectively, in 1983-84. This provides little evidence either for or against the presence of a survival rate advantage for fish rearing in side channels. It should be noted, however, that juvenile coho residing in off-channel habitats were observed to grow more during winter than those in the main channel, which might provide survival advantages later in life (Hartman et al., 1998). This possible advantage to rearing in side channels was not incorporated into the current model.

It is also thought that off-channel habitat may provide more stable smolt production to a system, with these parts of a system providing a larger portion of smolts migrating to sea during years with extreme flow conditions (Brown and Hartman, 1988). This has also been cited as a potential benefit of groundwater-fed side channels for coho 
(Sheng et al., 1990). However, the model does not take account of such benefits because the model is deterministic.

Extensive data are available on average coho smolt abundance from both wild habitat and side channels (reviewed in Bradford et al., 1997), however, these data may contain cases in which streams are not fully-seeded, hence, these data were not used to determine maximum smolt production per unit area of wild habitat. Instead, I used Baranski (1989), which found an average value of smolts per $\mathrm{m}^{2}$ of low-flow wetted area in ten Puget Sound streams of 0.18 . In that study, abundance of outmigrating smolts was not found to be correlated with escapenent estimates, which suggests these streams were fully seeded. Hence, I assumed that MaxSmoltsw $=0.18 \mathrm{~m}^{-2}$.

Data on smolt outmigration by wetted area is also available for side channels. Sheng et al. (1990) reported average smolt production estimates of 2.0 smolts per $\mathrm{m}^{2}$ from the Upper Paradise Channel. Lower smolt production estimates were obtained from the Worth Creek and Mamquam channels, possibly due to the lack of cover provided in the Worth Creek channel and drought conditions which dried up the Mamquam channel during some years (Sheng et al., 1990). In the future, expert judgement may be needed to supplement the available data because much of it is collected without knowledge of whether streams are fully seeded. As an example, Sheng (1992) reports outmigration of 0.5 smolts per $\mathrm{m}^{2}$ from a side channel in Dutenu Creek in the interior of B.C. (only 1 year of data), but suggests that the system was under-seeded, and that the true maximum number of smolts produced is 3 smolts per $\mathrm{m}^{2}$ of side channel area. In the model, I have assumed a maximum number of smolts produced per $\mathrm{m}^{2}$ (MaxSmolts $s_{E}$ ) of 2.0 . 


\section{Marine Survival Rate and Recruitment}

As stated above, spawner recruitment for each generation is calculated by multiplying the total number of coho smolts by the marine survival rate. Bradford (1995) reports an average marine survival rate from reviewed literature of approximately II percent. However, one of the major reasons for the decline of B.C. coho stocks has been low marine survival in recent years (Bradford, 1998; Walters, 1992). Coronado and Hilborn (1998) analyzed the spatial and temporal patterns in smolt to adult survival rates for hatchery and wild coho salmon in the Pacific Northwest between 1971 and 1990. Their analysis showed a marked decline in survival rates in British Columbia since the 1970s with survival rates dropping below 5 percent by the late 1980s. In light of this evidence, it seems appropriate to use a lower marine survival rate in my analysis. Hence, I set the marine survival rate to 3 percent, which is closer to recent values.

\section{Baseline Assumptions of the Deterministic Biological Simulation}

Baseline parameter values for the deterministic biological model are summarized in Table 1. Below, I discuss some of the model assumptions not already dealt with in the description of the model and the different scenarios that were investigated.

I simulated the dynamics of a hypothetical coho population for 10 generations. It was assumed that the coho population inhabited a $10-\mathrm{km}$-long stream averaging $5 \mathrm{~m}$ in width (i.e. WildArea $=50,000 \mathrm{~m}^{2}$ ) with 20 percent of the total area being suitable for spawning (SpawnArea $=10,000 \mathrm{~m}^{2}$ ). I simulated responses of the coho population to the construction of a $2,000 \mathrm{~m}^{2}$ side channel. With the above conditions, the side channel constituted Sidearea $/($ SideArea + SpawnArea $)=16.7$ percent of total spawning habitat 
Table 1. Baseline parameter values used in the biological simulation model and calculation of the net present value of groundwater-fed side channels.

\begin{tabular}{|c|c|c|}
\hline Value & Name & Description \\
\hline 50 & initial & Number of coho recruits for first three years \\
\hline 0.5 & pf & Proportion females \\
\hline $\mathbf{l}$ & alpha & Preference for enhanced spawning habitat ( $l=$ no preference) \\
\hline 1 & beta & Preference for enhanced rearing habitat ( $1=$ no preference) \\
\hline 2000 & sidearea & Area of side channels $\left(\mathrm{m}^{2}\right)$ \\
\hline 10000 & spawnarea & Natural spawning area $\left(\mathrm{m}^{2}\right)$ \\
\hline 50000 & wildarea & Natural rearing area $\left(\mathrm{m}^{2}\right)$ \\
\hline 2500 & fec & Fecundity (i.e. eggs per female) for coho \\
\hline 0.2 & $\mathbf{a}$ & Wild egg-to-fry survival for coho \\
\hline 0.172 & b & Wild density independent fry-to-smolt survival for coho \\
\hline 0.5 & prop_incl & Proportional increase in coho egg-to-fry survival rate \\
\hline 0.5 & prop_inc2 & Proportional increase in coho fry-to-smolt survival rate \\
\hline 0.5 & prop_inc3 & Proportional increase in chum egg-to-fry survival rate \\
\hline 2 & $\max E$ & Maximum smolts per $\mathrm{m}^{2}$ in enhanced \\
\hline 0.18 & $\max W$ & Maximum smolts per $\mathrm{m}^{2}$ in wild \\
\hline 0.03 & marine & Marine survival rate for coho \\
\hline 3.5 & kgpercoho & Average weight per adult coho (in $\mathrm{kg}$ ) \\
\hline 200 & chumperm2 & Chum fry produced per $\mathrm{m} 2$ of side channel \\
\hline 0.007 & chum_marine & Marine survival rate for chum \\
\hline 3 & kgperchum & Average weight per adult chum (in $\mathrm{kg}$ ) \\
\hline $5 \%$ & disc & Real discount rate \\
\hline 23 & lifetime & $\begin{array}{l}\text { Number of years over which side channel costs and benefits } \\
\text { accrue }\end{array}$ \\
\hline$\$ 35.98$ & capitalcost & Construction costs per m2 of side channel (in 1998 dollars) \\
\hline$\$ 19.66$ & sportben & Benefit per fish of coho caught in sport fishery (1998 dollars) \\
\hline$\$ 3.05$ & comben & $\begin{array}{l}\text { Benefit per } \mathrm{kg} \text { of fish of coho caught in commercial fishery } \\
\text { (1998 dollars) }\end{array}$ \\
\hline 0.5 & propSP & Proportion of coho harvest to sport fishery \\
\hline 0.5 & propCOM & Proportion of coho harvest to commercial fishery \\
\hline$\$ 1.82$ & chumben & $\begin{array}{l}\text { Benefit per } \mathrm{kg} \text { for coho caught in commercial fishery ( } 1998 \\
\text { dollars) }\end{array}$ \\
\hline
\end{tabular}




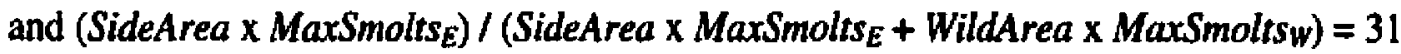
percent of total smolt rearing capacity for the stock.

The relative importance of a groundwater-fed side channel for spawning and rearing depends upon the mix of habitat available to a coho population. For example, in a system with limited spawning gravel but abundant pool habitat, a side channel would constitute a large proportion of the total (wild + enhanced) spawning habitat, but only a small proportion of the total rearing habitat. In a system with little existing off-channel habitat, a side channel would also constitute a large proportion of rearing habitat as well (in units of fish potentially produced), because off-channel habitats generally contribute a disproportionately large number of smolts produced in a system (Brown and Hartman, 1988; Decker, 1998). Obviously, the mix of habitats is important when considering the benefits of groundwater-fed side channels, because the proportion of fish affected by side channels at each life stage is dependent upon availability of habitat.

The maximum number of smolts produced by the population without the side channel is (WildArea $\times$ MaxSmoltsw) $=9,000$ smolts. Hence, the number of spawners required to achieve full-seeding of the wild system is $9,000 /(P f \times F e c u n d i t y \times a \times b)=$ 210. I set initial spawner abundance to be 50 , which is approximately 25 percent of the number required to fully seed the wild system.

I simulated four different scenarios with respect to the presence/absence of a side channel and the presence/absence of "incubation" and "rearing effects" (i.e. incremental increases in survival rates from the egg-to-fry and fry-to-smolt life stages). Specifically, the following scenarios were investigated:

- Scenario 1: Baseline Scenario - There is no side channel. 
- Scenario 2: Side channel with incubation and rearing effects present - The progeny of spawners using side channels have a 50 percent increase in their egg-to-fry survival rate and juveniles rearing in the side channel show a 50 percent increase in their density-independent fry-to-smolt survival rate. From equations (8) and (11), these translate into values for $\theta_{l}$ and $\theta_{2}$ of 0.405 .

- Scenario 3: Side channel without incubation effects present - In this scenario, $\theta_{l}$ is set to 0 , while $\theta_{2}$ is 0.405 .

- Scenario 4: Side channel without rearing effects present - In this scenario, $\theta_{1}$ is set to 0.405 , while $\theta_{2}$ is 0 .

Comparison of these scenarios serves to answer a number of research questions:

(1) Differences between scenarios 1 and 2 show the extent to which groundwater-fed side channels contribute to the rebuilding of depressed coho stocks under the optimistic assumptions stated above (i.e. a 50 percent increase in both egg-to-fry and fry-to-smolt survival rates for fish using side channels during these respective life-stages).

(2) Comparison of scenarios 3 and 4 with scenario I illustrates whether effects at the incubation phase or rearing phase of coho life histories is more important to stock rebuilding. This question is important for a number of reasons. First, it indicates which variables are more important for monitoring stock rebuilding benefits of side channels. [If one were dealing with wild systems that were fully seeded, the benefits of adult production from groundwater-fed side channels would be more important, because without channels, higher escapements would have no effect on adult recruits to the population because habitat capacity is the limiting factor. In this case, monitoring should be focused on the maximum smolt production achievable from side channels, rather than 
life-stage specific increases in survival rates.] Second, these comparisons will indicate which uncertain parameters have a larger effect on recruitment to the stock, which could affect the decision analysis stage of the project. For example, if stock rebuilding is much more sensitive to effects at one life stage, then only one "effect size" needs to be explicitly treated as an uncertain state of nature in the decision analysis.

\section{Sensitivity Analysis}

Because the biological model in this initial analysis was deterministic, I performed a sensitivity analysis to see how varying the magnitude of incubation $\left(\theta_{l}\right)$ and rearing $\left(\theta_{2}\right)$ effects altered the degree to which the hypothetical coho stock was able to rebuild. The minimum and maximum values for $\theta_{l}$ and $\theta_{2}$ were 0 and 0.693 respectively, which translate into 0 percent and 100 percent increases in the egg-to-fry and fry-to-smolt survival rates for coho salmon utilizing the side channels.

To test the sensitivity of results to the habitat assumptions, I repeated these two sensitivity analyses with different values for the area parameters to reflect cases in which side channels represent a larger portion of total spawning habitat. Thus, I assumed that only 10 percent (rather than 20 percent) of the total area was suitable for spawning (i.e. SpawnArea $=5,000 \mathrm{~m}^{2}$ ). Under this new assumption, the side channel constitutes $2000 /(2,000+5,000)=28.6$ percent (rather than 16.7 percent) of total spawning habitat.

One of the most commonly measured indicators for the benefits of side channels for coho salmon is smolt outmigration from side channels. However, this measure may not accurately reflect incremental smolt production in a system if the system is not fullyseeded. I used the biological model to compare smolt outmigration from a side channel 
with true incremental smolt production resulting from enhancement for various levels of effect sizes, spawner abundances, and habitat preferences for coho juveniles. The results of this analysis illustrate the conditions under which smolt outmigration from channels is, or is not, a good indicator of incremental smolt production in a system.

\section{RESULTS AND DISCUSSION}

\section{Baseline Results}

For all three cases with enhancement, smolt production in each generation increased more rapidly than the case without the side channel (Figure 4). Furthermore, the difference between smolt production each generation in the cases with the side channel and the case without the side channel increased over time until the population reached full-seeding ( 13,000 smolts with the side channel and 9,000 smolts without the side channel). For all scenarios with enhancement, average smolt production for the first 3,5 , and 10 generations was substantially greater than the case without enhancernent and smolt abundance reached the wild fully-seeded level after fewer generations (Table 2).

The faster increase in smolt production in scenario 3 (no "incubation effect") than scenario 4 (no "rearing effect") indicates that effects of enhancement at the fry-to-smolt life-stage may be more important than effects at the egg-to-fry life-stage when the magnitudes of these two effects are equal. This result occurred because the side channel comprised a larger percentage of total rearing habitat in the system than total spawning habitat under my baseline assumptions.

The abundance of smolts in Table 2 is a direct indicator of adult abundance because I assumed a constant marine survival rate. These adults can be further 
Figure 4. Simulated smolt production over 10 generations for four model scenarios. See text for details.

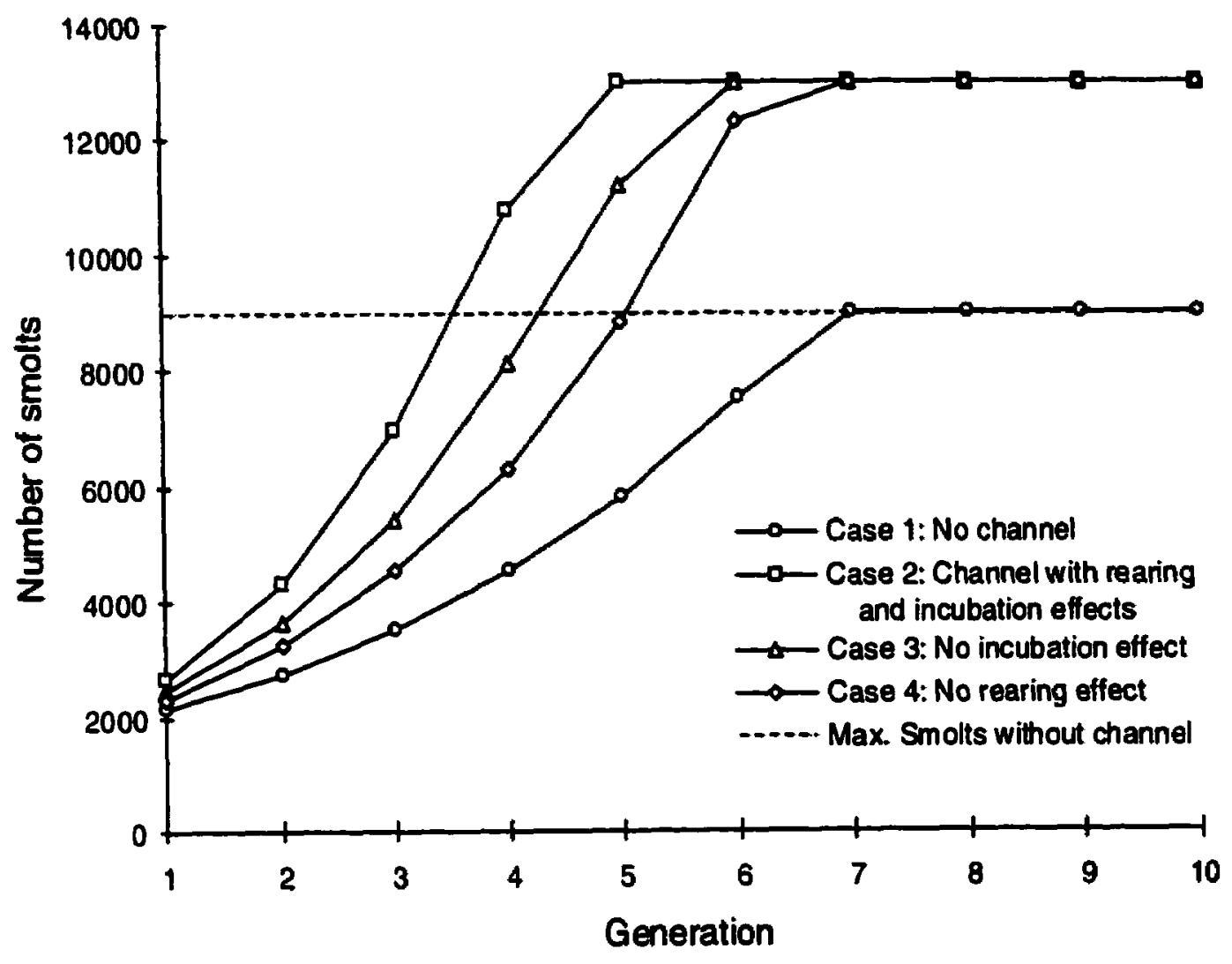


Table 2. Summary statistics for baseline simulations of the biological model under four scenarios with respect to the presence/absence of a groundwater-fed side channel and effects on "incubation" (egg-to-fry survival rate) and "rearing" (fry-to-smolt survival rate).

Summary Statistic

Average annual smolt production for:

First 3 generations

First 5 generations

First 10 generations

First generation in which full seeding occurs

\section{Scenario}

Case 1: No Case 2: Case 3: Case 4:

side channel With side No No rearing channel incubation effect effect

$\begin{array}{llll}2,801 & 4,650 & 3,859 & 3,373\end{array}$

$3,756 \quad 7,544 \quad 6,186 \quad 5,051$

$\begin{array}{llll}6,233 & 10,272 & 9,593 & 8,960\end{array}$

$\begin{array}{llll}7 & 4 & 5 & 6\end{array}$


translated into incremental adults, i.e. those additional adults arising from side channels, which can be used for evaluating management options along with rebuilding rate.

\section{Sensitivity Analysis}

Average annual smolt production over the first 5 generations and the generation in which smolt abundance reached the fully-seeded level of the wild system were both sensitive to changes in both $\theta_{1}$ and $\theta_{2}$ (Tables 3 and 4). However, both of these measures of rebuilding were slightly more sensitive to changes in survival rates at the fry-to-smolt life-stage (i.e. changes in $\hat{6}_{2}$ ), than changes in survival rates at the egg-to-fry life-stage (i.e. changes in $\theta_{l}$ ). For example, where $\theta_{1}=0$, increasing $\theta_{2}$ from 0 to 0.693 (a 100 percent increase in the fry-to-smolt survival rate for coho rearing in side channels) increased average annual smolt production by 111 percent and reduced the generation in which the system reached the wild fully-seeded level from 7 to 4 (Tables 3 and 4). Conversely, where $\theta_{2}=0$, increasing $\theta_{l}$ from 0 to 0.693 (a 100 percent increase in the egg-to-fry survival rate for coho incubating in side channels) increased average annual smolt production by 79 percent and reduced the generation in which the system reaches the wild fully-seeded level from 7 to 5 .

However, when I assumed instead that only 10 percent of the total wild area was suitable for spawning (rather than 20 percent as was assumed in the baseline scenario), both average smolt abundance for the first 5 generations and the year in which fullseeding first occurs are equally sensitive to changes in $\theta_{1}$ and $\theta_{2}$ (Tables 5 and 6). Where $\theta_{l}=0$, increasing $\theta_{2}$ from 0 to 0.693 increased average annual smolt production by 111 percent and reduced the generation in which full-seeding first occurs from 7 to 4 generations. Similarly, average annual smolt production increased by 114 percent and the 
Table 3. Average annual smolt abundance over first 5 generations for various combinations of incubation and rearing effects assuming the groundwater-fed side channel comprises 16.7 percent of total spawning habitat. $\theta_{1}$ and $\theta_{2}$ refer to the effects of enhancement on coho egg-to-fry and fry-to-smolt survival rates, respectively, as defined by equations (6) and (11).

\begin{tabular}{|c|c|c|c|c|c|c|c|c|c|c|c|}
\hline \multirow{2}{*}{$\begin{array}{c}\text { Rearing } \\
\text { Effect } \\
\left(\theta_{2}\right)\end{array}$} & \multicolumn{11}{|c|}{ Incubation Effect $\left(\theta_{l}\right)$} \\
\hline & 0.000 & 0.095 & 0.182 & 0.262 & 0.336 & 0.405 & 0.470 & 0.531 & 0.588 & 0.642 & 0.693 \\
\hline 0.000 & 3,756 & 4,010 & 4,253 & 4,577 & 4,778 & 5,051 & 5,433 & 5,677 & 6,016 & 6,289 & 6,706 \\
\hline 0.095 & 4,225 & 4,511 & 4,752 & 5,047 & 5,431 & 5,675 & 6,021 & 6,330 & 6,663 & 6,869 & 7,126 \\
\hline 0.182 & 4,697 & 5,034 & 5,287 & 5,662 & 6,015 & 6,249 & 6,610 & 6,869 & 7,126 & 7,266 & 7,534 \\
\hline 0.262 & 5,152 & 5,620 & 5,984 & 6,190 & 6,550 & 6,813 & 7,118 & 7,263 & 7,530 & 7,645 & 7,866 \\
\hline 0.336 & 5,715 & 6,114 & 6,437 & 6,697 & 7,103 & 7,251 & 7,471 & 7,597 & 7,818 & 8,040 & 8,163 \\
\hline 0.405 & 6,186 & 6,610 & 6,948 & 7,173 & 7,419 & 7,544 & 7,715 & 7,985 & 8,110 & 8,308 & 8,434 \\
\hline 0.470 & 6,681 & 7,091 & 7,318 & 7,438 & 7,660 & 7,789 & 8,039 & 8,238 & 8,378 & 8,605 & 8,674 \\
\hline 0.531 & 7,078 & 7,366 & 7,589 & 7,728 & 7,931 & 8,072 & 8,305 & 8,587 & 8,664 & 8,807 & 8,906 \\
\hline 0.588 & 7,380 & 7,605 & 7,848 & 7,993 & 8,239 & 8,453 & 8,625 & 8,753 & 8,819 & 8,919 & 9,018 \\
\hline 0.642 & 7,664 & 7,863 & 8,111 & 8,255 & 8,467 & 8,696 & 8,764 & 8,864 & 8,974 & 9,030 & 9,132 \\
\hline 0.693 & 7,920 & 8,169 & 8,350 & 8,451 & 8,641 & $\mathbf{8 , 8 0 7}$ & 8,875 & 8,985 & 9,087 & 9,155 & 9,259 \\
\hline
\end{tabular}


Table 4. Generation in which hypothetical coho population is first fully seeded assuming the groundwater-fed side channe] comprises 16.7 percent of total spawning habitat. $\theta_{1}$ and $\theta_{2}$ refer to the effects of enhancement on coho egg-to-fry and fryto-smolt survival rates, respectively, as defined by equations (6) and (11).

\begin{tabular}{|c|c|c|c|c|c|c|c|c|c|c|c|}
\hline \multirow{2}{*}{$\begin{array}{c}\text { Rearing } \\
\text { Eriect } \\
\left(\theta_{2}\right)\end{array}$} & \multicolumn{11}{|c|}{ Incubation Effect $\left(\theta_{l}\right)$} \\
\hline & 0.000 & 0.095 & 0.182 & 0.262 & 0.336 & 0.405 & 0.470 & 0.531 & 0.588 & 0.642 & 0.693 \\
\hline 0.000 & 7 & 7 & 6 & 6 & 6 & 6 & 5 & 5 & 5 & 5 & 5 \\
\hline 0.095 & 6 & 6 & 6 & 6 & 5 & 5 & 5 & 5 & 5 & 4 & 4 \\
\hline 0.182 & 6 & 6 & 5 & 5 & 5 & 5 & 5 & 4 & 4 & 4 & 4 \\
\hline 0.262 & 5 & 5 & 5 & 5 & 5 & 4 & 4 & 4 & 4 & 4 & 4 \\
\hline 0.336 & 5 & 5 & 5 & 5 & 4 & 4 & 4 & 4 & 4 & 4 & 4 \\
\hline 0.405 & 5 & 5 & 4 & 4 & 4 & 4 & 4 & 4 & 4 & 4 & 4 \\
\hline 0.470 & 4 & 4 & 4 & 4 & 4 & 4 & 4 & 4 & 4 & 3 & 3 \\
\hline 0.531 & 4 & 4 & 4 & 4 & 4 & 4 & 4 & 4 & 3 & 3 & 3 \\
\hline 0.588 & 4 & 4 & 4 & 4 & 4 & 4 & 3 & 3 & 3 & 3 & 3 \\
\hline 0.642 & 4 & 4 & 4 & 4 & 4 & 3 & 3 & 3 & 3 & 3 & 3 \\
\hline 0.693 & 4 & 4 & 4 & 4 & 3 & 3 & 3 & 3 & 3 & 3 & 3 \\
\hline
\end{tabular}


Table 5. Average annual smolt abundance over first 5 generations for various combinations of incubation and rearing effects assuming the groundwater-fed side channel comprises 28.6 percent of total spawning habitat. $\theta_{1}$ and $\theta_{2}$ refer to the effects of enhancement on coho egg-to-fry and fry-to-smolt survival rates, respectively, as defined by equations (6) and (11).

\begin{tabular}{|c|c|c|c|c|c|c|c|c|c|c|c|}
\hline \multirow{2}{*}{$\begin{array}{c}\text { Rearing } \\
\text { Efifect } \\
\left(\theta_{2}\right)\end{array}$} & \multicolumn{11}{|c|}{ Incubation Effect $\left(\theta_{i}\right)$} \\
\hline & 0.000 & 0.095 & 0.182 & 0.262 & 0.336 & 0.405 & 0.470 & 0.531 & 0.588 & 0.642 & 0.693 \\
\hline 0.000 & 3,756 & 4,215 & 4,658 & 5,123 & 5,653 & 6,217 & 6,802 & 7,066 & 7,402 & 7,798 & 8,019 \\
\hline 0.095 & 4,225 & 4,672 & 5,141 & 5,678 & 6,307 & 6,825 & 7,146 & 7,435 & 7,854 & 8,184 & 8,400 \\
\hline 0.182 & 4,697 & $5,15]$ & 5,695 & 6,307 & 6,848 & 7,169 & 7,515 & 7,908 & 8,216 & 8,440 & 8,641 \\
\hline 0.262 & 5,152 & 5,721 & 6,273 & 6,825 & 7,205 & 7,549 & 7,895 & 8,204 & 8,460 & 8,698 & 8,853 \\
\hline 0.336 & 5,715 & 6,233 & 6,783 & 7,215 & 7,523 & 7,867 & 8,176 & 8,493 & 8,713 & $\mathbf{8 , 8 7 3}$ & 9,047 \\
\hline 0.405 & 6,186 & 6,735 & 7,216 & 7,546 & 7,954 & 8,266 & 8,588 & 8,784 & 8,947 & 9,081 & 9,261 \\
\hline 0.470 & 6,681 & 7,183 & 7,509 & 7,916 & 8,228 & 8,593 & 8,792 & 8,924 & $\mathbf{9 , 0 7 0}$ & 9,260 & 9,390 \\
\hline 0.531 & 7,078 & 7,421 & 7,874 & 8,186 & 8,565 & 8,766 & 8,899 & 9,045 & 9,232 & 9,387 & 9,533 \\
\hline 0.588 & 7,380 & 7,713 & 8,140 & 8,516 & 8,738 & 8,871 & 9,018 & 9,203 & 9,358 & 9,516 & 9,766 \\
\hline 0.642 & 7,664 & 7,973 & 8,434 & 8,701 & 8,841 & 8,989 & 9,171 & 9,327 & 9,570 & 9,733 & 9,900 \\
\hline 0.693 & 7,920 & 8,259 & 8,571 & 8,810 & 8,958 & 9,137 & 9,294 & 9,534 & 9,698 & 9,865 & 10,034 \\
\hline
\end{tabular}


Table 6. Generation in which hypothetical coho population is first fully seeded assuming the groundwater-fed side channel comprises 28.6 percent of total spawning habitat, $\theta_{1}$ and $\theta_{2}$ refer to the effects of enhancement on coho egg-to-fry and fryto-smolt survival rates, respectively, as defined by equations (6) and (11).

\begin{tabular}{|c|c|c|c|c|c|c|c|c|c|c|c|}
\hline \multirow{2}{*}{$\begin{array}{c}\text { Rearing } \\
\text { Efrect } \\
\left(\theta_{2}\right)\end{array}$} & \multicolumn{11}{|c|}{ Incubation Effect $\left(\theta_{l}\right)$} \\
\hline & 0.000 & 0.095 & 0.182 & 0.262 & 0.336 & 0.405 & 0.470 & 0.531 & 0.588 & 0.642 & 0.693 \\
\hline 0.000 & 7 & 6 & 6 & 5 & 5 & 5 & 5 & 4 & 4 & 4 & 4 \\
\hline 0.095 & 6 & 6 & 5 & 5 & 5 & 5 & 4 & 4 & 4 & 4 & 4 \\
\hline 0.182 & 6 & 5 & 5 & 5 & 4 & 4 & 4 & 4 & 4 & 4 & 3 \\
\hline 0.262 & 5 & 5 & 5 & 4 & 4 & 4 & 4 & 4 & 4 & 3 & 3 \\
\hline 0.336 & 5 & 5 & 4 & 4 & 4 & 4 & 4 & 4 & 3 & 3 & 3 \\
\hline 0.405 & 5 & 4 & 4 & 4 & 4 & 4 & 4 & 3 & 3 & 3 & 3 \\
\hline 0.470 & 4 & 4 & 4 & 4 & 4 & 4 & 3 & 3 & 3 & 3 & 3 \\
\hline 0.531 & 4 & 4 & 4 & 4 & 4 & 3 & 3 & 3 & 3 & 3 & 3 \\
\hline 0.588 & 4 & 4 & 4 & 4 & 3 & 3 & 3 & 3 & 3 & 3 & 3 \\
\hline 0.642 & 4 & 4 & 4 & 3 & 3 & 3 & 3 & 3 & 3 & 3 & 3 \\
\hline 0.693 & 4 & 4 & 3 & 3 & 3 & 3 & 3 & 3 & 3 & 3 & 3 \\
\hline
\end{tabular}


generation in which first seeding occurred was reduced from 7 to 4 generations where $\theta_{2}$ $=0$ and $\theta_{1}$ was increased from 0 to 0.693 .

When the stream was fully-seeded (180 female spawners), measured smolt outmigration from the side channel equaled incremental smolt production (i.e. additional smolts due to enhancement) (right side of panels A through D in Figure 5). However, when the population was at only half this level ( 90 spawners), smoit outmigration from channels greatly overestimated true incremental smolt production (left side of panels A through $D$ in Figure 5). In the extreme case, where side channels provided no survival benefits (i.e. when $\theta_{l}$ and $\theta_{2}=0$ ), the number of smolts from the channel was still positive, even though no incremental smolts were produced due to enhancement (Figures 5B, 5D). The reason for these results is that many of the fish using the side channel would have used wild habitat if the side channel had not been constructed and, when the system is not fully seeded, some of these fish would have survived to become smolts.

Rearing preferences also affected the relationship between incremental smolts and smolt outmigration. In the model, where $\beta=1$, the number of coho rearing in each habitat type is proportional to the rearing capacity of each habitat type (Figures 5A and 5B). Where it is assumed that $\beta>1$ (Figures 5C and 5D), a disproportionate number of juveniles rear in side channels. In this latter case, the difference between measured smolt outmigration and incremental smolts when the system is not fully-seeded was exacerbated.

The number of smolts produced by the system with the channel increased more quickly with increasing spawner abundance than it did without the channel (Figure 6). Hence, at higher spawner abundances, incremental smolt production was greater than at 

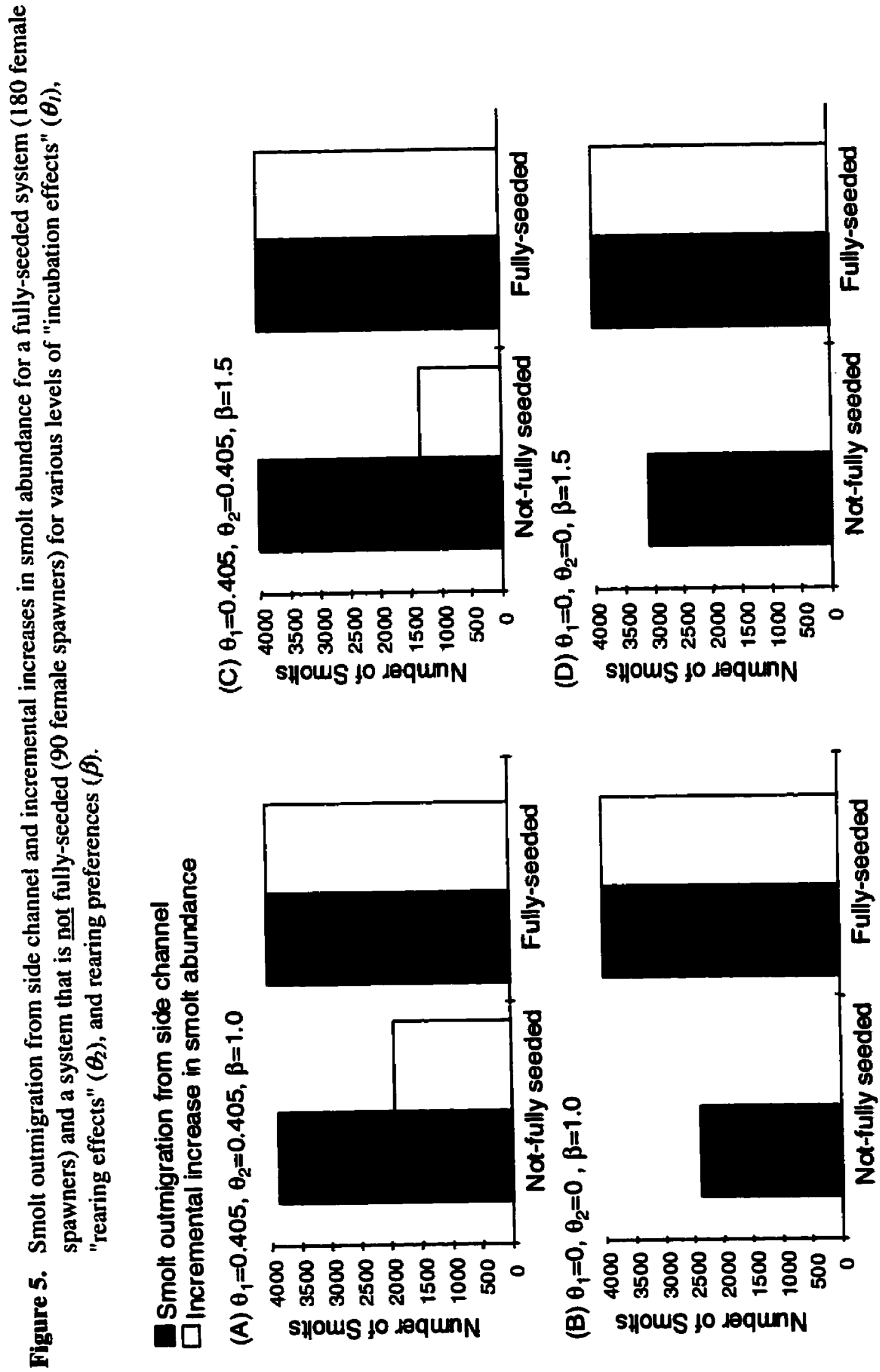
Figure 6. Smolts produced by the system with and without side channels as a function of female spawner abundance for baseline parameter values.

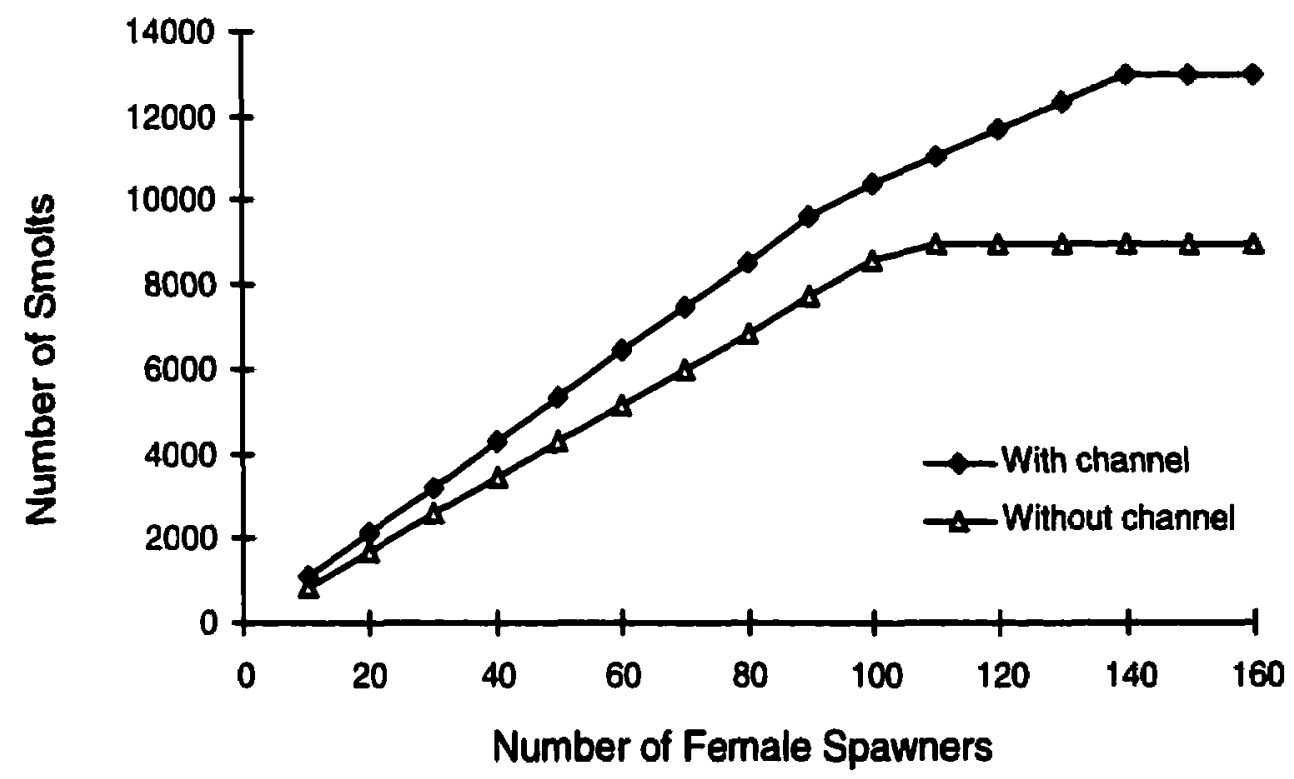

Figure 7. Smolt outmigration and incremental smolt production as a function of female spawner abundance for baseline parameter values.

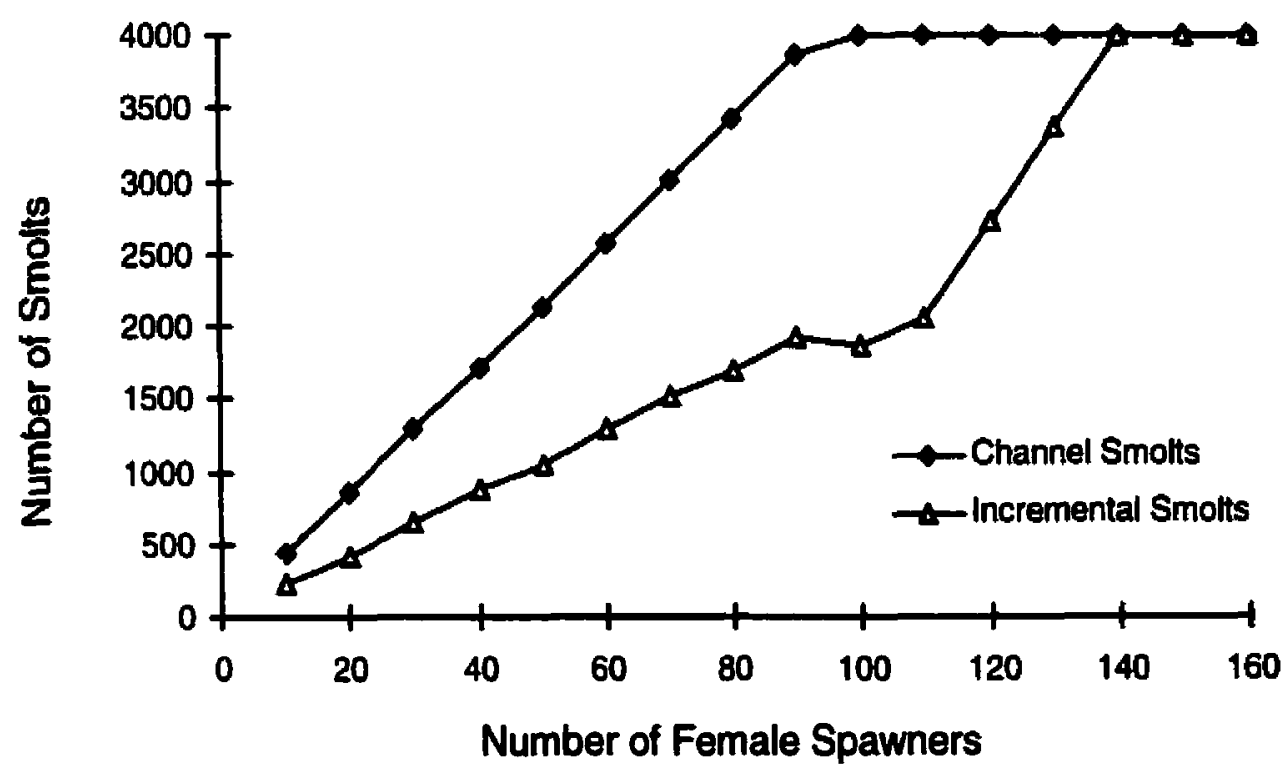


lower spawner abundances. However, incremental smolt production did not increase as quickly as smoit outmigration from channels (Figure 7). Incremental smolt production only approached smolt outmigration from channels when the system approached fullseeding.

\section{GENERAL DISCUSSION}

Analysis of the deterministic biological model has shown that the rebuilding of depressed coho stocks may be facilitated through use of groundwater-fed side channels. However, the importance of "effect size" at each life-stage is contingent upon relative amounts of available habitat. For example, when a side channel comprises a small proportion of total rearing habitat, but a large proportion of total spawning habitat, the incremental increase in egg-to-fry survival rates for coho salmon using the side channel may be the key biological effect that should be monitored. However, the converse is true when a side channel is only a small portion of total spawning habitat, but a large proportion of rearing habitat. For the range of habitat conditions I simulated, effects at both life-stages potentially have a large effect on stock rebuilding. Owing to uncertainty in the magnitude of life-stage specific survival advantages conferred to coho salmon using side channels, the degree to which rebuilding may be facilitated is unknown.

One problem that hampered this analysis was the lack of good quality data to estimate parameters for the model. While data were available on the egg-to-fry survival rate of the progeny of coho spawning in side channels, it is difficult to estimate the incremental benefits to coho populations in those cases (in terms of increased survival rates) because no data on survival rates from nearby wild areas was collected at the same 
time. Comparison of channel data with values from the literature may be misleading because many factors (such as inherent differences between stocks, differences in spawner density, differences between years, etc.) confound estimates of effect size of channels that might be obtained by simply taking the difference between average survival rates for wild coho from the literature and average survival rates of channel-incubating fish. Also, data on fry-to-smolt survival rate for channel-rearing coho were not available, hence, not even simple estimates of the incremental increase in fry-to-smolt survival could be obtained.

The most commonly used performance measures for habitat enhancement projects for coho salmon and other stream-dwelling salmonids are estimates of abundance in enhanced areas. Such simple estimates of abundance are not sufficient to determine possible mechanisms that could lead to changes in abundance or biomass as a result of habitat enhancement (Riley and Fausch, 1995). In particular, movement may be an important demographic response to habitat enhancement in stream-dwelling salmonids (Gowan, 1994). Gowan and Fausch (1996) undertook a detailed study of trout responses to habitat enhancement in 6 Colorado streams. Trout abundance was measured both before and after installation of log-drop structures in control and treatment reaches within each of the 6 streams. To distinguish between the effects of movement and survival, fish caught in the treatment and control reaches were marked. While abundance in treatment reaches increased relative to controls after enhancement, there was little evidence to support the hypothesis that the increase in abundance was due to an increase in survival rates. Due to the large number of unmarked fish caught in later samples, they concluded that relative changes in abundance were primarily caused by immigration of fish from 
outside the study reaches. However, increased abundance in enhanced reaches could be indicative of increased survival on a larger spatial scale (i.e. for the entire stream) if fish moving into enhanced areas would have died if they had not encountered the enhanced habitat, or if the habitat vacated by immigrants was suitable, which allowed subordinate fish to use that habitat (Gowan and Fausch, 1996). Since there was little fishing pressure in all 6 streams (Riley and Fausch, 1995), increased abundance in enhanced reaches probably reflected increase habitat capacity for these populations.

Thus, another important consideration in the choice of appropriate performance measures for evaluating habitat enhancement is the issue of capacity versus productivity. Measures of abundance in enhanced habitat may provide information on the effect that enhancement may have on the capacity of a habitat, if habitat is the limiting factor for that population. However, such simple measures of abundance do not adequately characterize the change in population dynamics associated with changes in habitat quality or quantity (Hayes et al. 1996). In particular, where habitat management decisions are being considered for populations at low abundance, productivity (i.e. the proportion of fish surviving between two life stages) is the key parameter of interest (Hayes et al. 1996; Mobrand et al. 1997). My analyses indicate that smolt outmigration from groundwaterfed side channels will not necessarily reflect the incremental increase in smolt abundance in a stock resulting from building side channels. In particular, smolt outmigration will overstate the true incremental increase in smolt abundance and understate the true capacity of enhanced habitat, if a stream is not fully seeded, because the fish utilizing the side channel would have used wild habitat if the side channel had not been constructed and some would still have survived to become smolts. 
These problems highlight the need to carefully choose appropriate performance measures for assessing the effects of habitat alterations. Jones et al. (1996) stress that the biological mechanisms through which habitat manipulations can achieve management objectives should be explicitly recognized. Appropriate indicators or performance measures for enhancement projects should be based on these hypothesized mechanisms and management objectives. In the case of groundwater-fed side channels for depressed coho stocks, rebuilding may be facilitated if the productivity of stocks can be improved through enhancement. Appropriate indicators for groundwater-fed side channels are the incremental increases egg-to-fry and fry-to-smolt survival rates for fish using side channels in underseeded streams. To obtain unconfounded estimates for the relevant biological effects of enhancement, survival rates should be monitored simultaneously in side channeis and in adjacent wild areas. 


\section{Decision analysis for determining the optimal experimental design}

\section{for programs to monitor groundwater-fed side channels}

\section{INTRODUCTION}

The magnitude of the biological effects of enhancement projects are often highly uncertain. Hence, it is often unclear whether the net benefits of enhancement are positive or negative (Figure 8) and, therefore, whether it is worthwhile to apply a particular method of enhancement more widely to other systems. As a result of this uncertainty, there may be benefits to monitoring. By monitoring the biological effects of enhancement, managers can improve investment decisions in enhancement projects by increasing the chances that enhancement will be applied more widely when, in fact, it does work sufficiently well to justify the costs (for example, if the net present value of enhancement (NPV) exceeds zero), and reducing the chances of undertaking enhancement when it does not (NPV $<0$ ). However, monitoring cannot ensure that the best possible outcome will always occur because of the many sources of variation that can influence the observed "effect size" $(\theta)$ of enhancement in any given system and year.

For instance, for multiple systems (sub-populations) and years, there will likely be three key sources of variability in $\theta$. First, there will be differences in $\theta$ from system to system across years (e.g., across years, the enhancement project in system $A$ is more effective than that in system $B$ ). Second, there may be differences in $\theta$ from year to year across systems (e.g., across systems, projects were more effective in year $t$ than in year $t+1)$. Third, within a given system there will be natural variation in $\theta$ (i.e., variability from year to year that is independent of other systems). In addition to these three sources 
Figure 8. A hypothetical linear relationship between the net present value of an enhancement project as a function of the magnitude of the biological effect of enhancement.

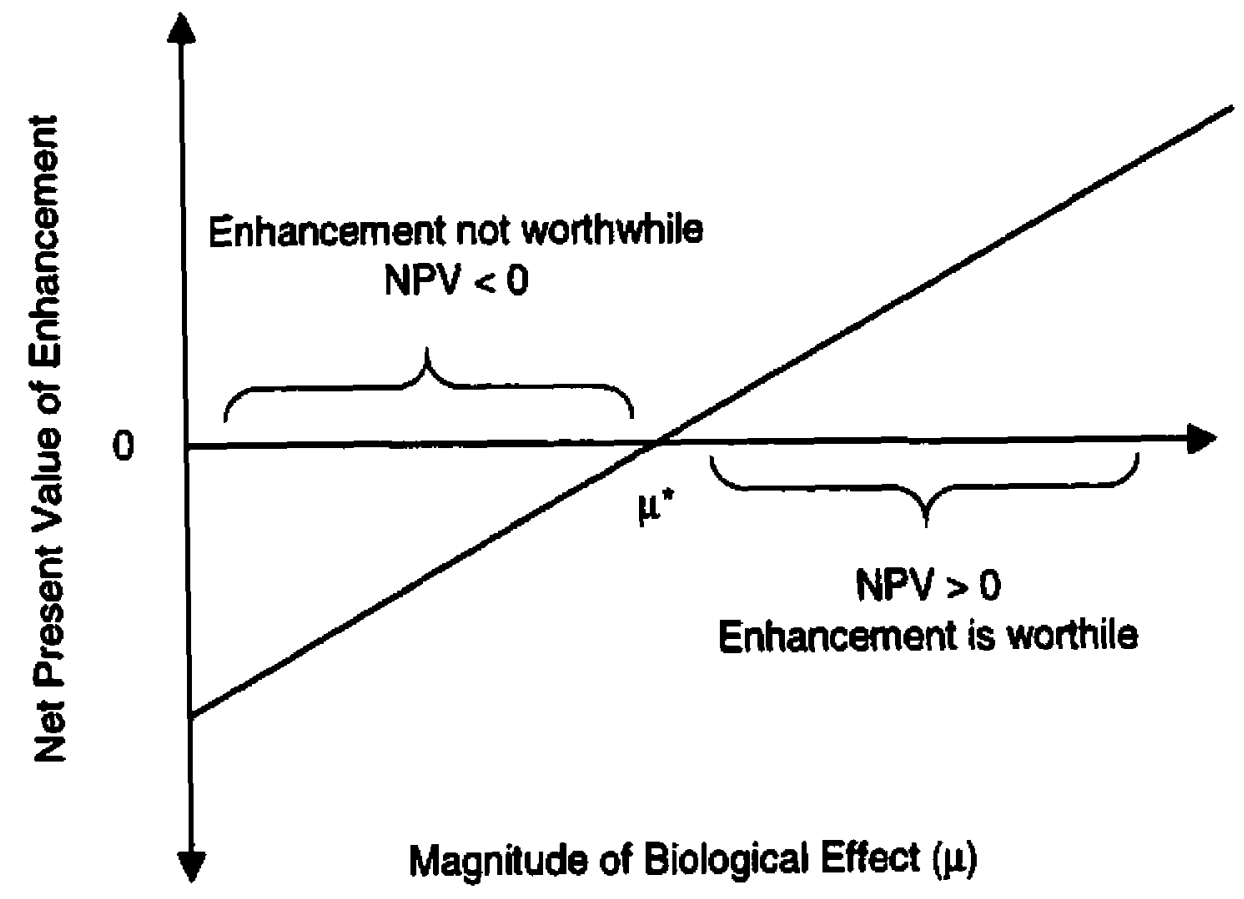


of variability in the true effect size $\theta$, an estimate of $\theta$ will also contain some measurement error. These sources of variability in estimated $\theta$ are defined as

$$
\hat{\theta}_{i t}=\mu+X_{i}+Y_{i}+e_{i t}+v_{i t}
$$

where $\hat{\theta}_{i r}$ denotes the estimated effect of enhancement for system $i$ in year $t, \mu$ denotes the mean effect across systems and years, $X_{i}$ denotes the system-specific effect (common to all years), $Y_{t}$ denotes the year-specific effect (common to all systems), $e_{i t}$ denotes the random variability in the effect of enhancement for system $i$ in year $t$, and $v_{i t}$ denotes the error in the measurement of that effect. I assumed that the variables $X, Y, e$, and $v$ are normally distributed with zero means and corresponding variances: $\sigma_{X}^{2}, \sigma_{Y}^{2}, \sigma_{e}^{2}$, and $\sigma_{v}^{2}$

From the standpoint of future decisions about using a particular type of enhancement, the most important component in equation (14) is the true mean effect size across years and across replicate systems, $\mu$. After a period of data collection, $\mu$ would be estimated as

$$
\hat{\mathrm{\mu}}=\frac{\sum_{i=1}^{N} \sum_{t=1}^{T} \hat{\theta}_{i t}}{N T},
$$

where $\mathbf{N}$ is the number of systems monitored and $\mathbf{T}$ is the number of years monitored. The variance of estimated mean effect of enhancement is:

$$
\sigma_{\tilde{\mu}}^{2}=\frac{\sigma_{x}^{2}}{N}+\frac{\sigma_{y}^{2}}{T}+\frac{\sigma_{e}^{2}+\sigma_{v}^{2}}{N T} .
$$

More precise estimates of $\mu$ will increase the chance of making correct decisions about whether to implement the enhancement method more widely. Thus, increasing the 
number of systems and years monitored or using more precise sampling methods (i.e. sampling methods with lower $\sigma_{v}^{2}$ ) can increase the chances that a correct management decision is made at the conclusion of any monitoring program. However, monitoring costs will also increase with the size of the experiment (i.e. number of subpopulations or systems) and with more precise sampling methods. Hence, there exists a tradeoff between the costs of obtaining information (i.e. the costs of monitoring) and the value of that information.

In this section, I develop a general framework for choosing among alternative monitoring programs used to evaluate enhancement projects for Pacific salmon. I then apply this framework to groundwater-fed side channels used by coho and chum salmon. The framework is based on decision analysis, a method that systematically evaluates different management alternatives while explicitly taking into account sources of uncertainty regarding the true state of nature (in this case, the effectiveness of enhancement) (Keeney !982). Several authors have used decision analysis to evaluate experimental management strategies in fisheries (Walters 1977,1986; Sainsbury 1988; McAllister and Peterman 1992; Robb and Peterman 1998; Walters and Green 1998). However, none of these examples apply this decision analysis approach to experimental management of enhancement projects.

In this analysis, I compare monitoring programs in terms of their expected value of net economic benefits. I also calculated outcomes for different management strategies in terms of the rebuilding of depressed coho stocks. This framework accounts for factors that a management agency can alter (e.g. whether to monitor at all and if so, the duration of the monitoring program, number of systems included in the monitoring program, and 
sampling methods used), as well as variation in factors that it does not control (e.g. yearly changes in survival rate within a stock due to natural environmental variation). By doing so, the framework can determine which actions best achieve management objectives.

\section{METHODS}

\section{The Decision Problem}

The decision tree (Figure 9) outlines the various management actions, uncertain states of nature, and possible outcomes. The management actions (emerging from the first square node of the decision tree) are alternative monitoring programs that vary in terms of the duration of the monitoring program, the number of systems monitored, and the sampling methods used to estimate the magnitude of the biological effects of enhancement. In this decision problem, emerging from the two sets of round nodes are the two uncertain states of nature. The first is the magnitude of the true mean effect size $(\mu)$ of the method of enhancement in question. The probabilities for different values of mean effect size (denoted $P_{1}, P_{2}, \ldots, P_{m}$ ) reflect the prior beliefs of managers regarding the efficacy of enhancement. The second uncertain state of nature is whether the estimated effect size $(\hat{\mu})$ is greater than or less than the critical effect size $\left(\mu^{*}\right)$, where the critical effect size is the magnitude of the biological effect of enhancement at which the net present value of an enhancement project is equal to zero (Figure 8). I assumed that managers would choose to apply the enhancement more widely if the estimated effect size is greater than or equal to the critical effect size and would not apply enhancement more widely if the estimated effect size is less than the critical value. Hence, a correct 
Figure 9. Decision tree for comparing monitoring programs. See text for details.

Management Options

Alternative monitoring designs (duration, number of systems, sampling method)

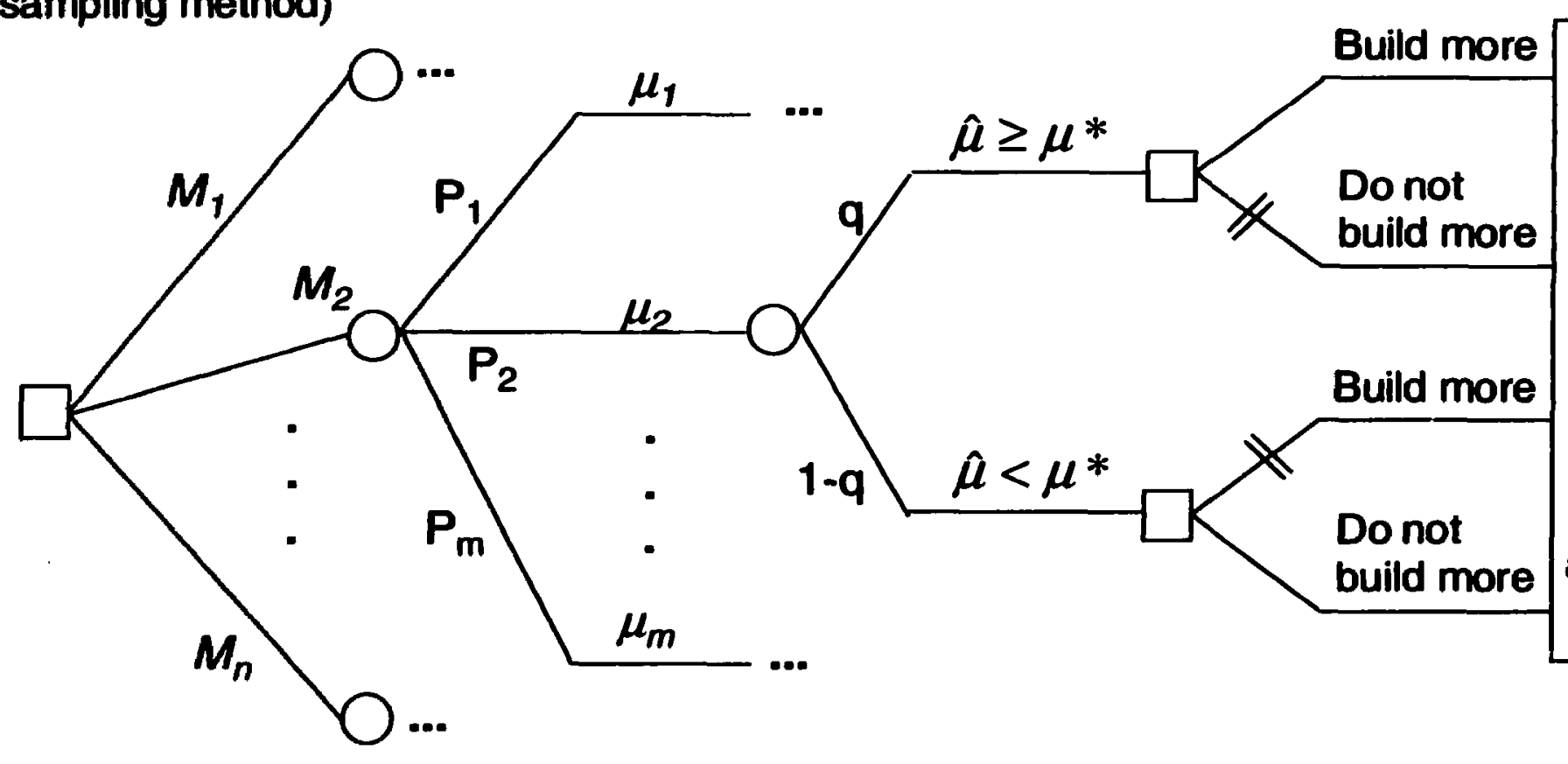

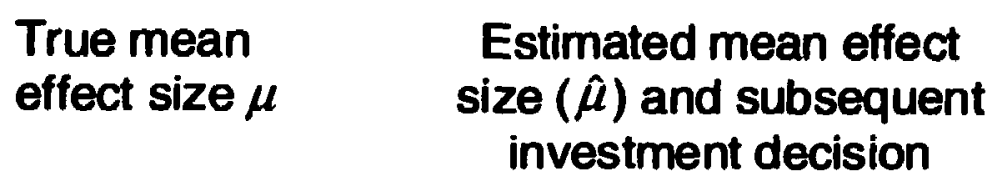

Estimated mean effect size $(\hat{\mu})$ and subsequent investment decision

True mean

effect size $\mu$

Uncertain States of Nature
Performance

Measures

(1) Net present value

of economic

benefits

(2) Measures of stock rebuilding (e.g. abundance of adults at time t) 
management decision results if both the true and estimated effect sizes are greater than or equal to the critical effect size or if both are less than the critical effect size. Conversely, managers would incorrectly choose to proceed with enhancement if the true effect size is less than the critical value but the estimated effect size is greater than the critical value. Similarly, managers would incorrectly choose not to proceed with enhancement if the true effect size is greater than or equal to the critical level while the estimated effect size is less than the critical level. The probability that monitoring leads to a correct management decision is dependent upon the true mean effect size and the monitoring program chosen. As the difference between the true mean effect size and the critical effect size grows, the probability of a correct decision increases. Increasing precision of monitoring programs (by adding more systems or years to the monitoring program and/or employing more precise sampling methods) also increases the probability of a correct decision. The possible outcomes (i.e. the net economic benefits and stock rebuilding measures) are dependent upon the true mean effect size and whether a correct investment decision was made. Alternative monitoring designs are ranked in terms of the expected values of these outcomes.

In order to calculate the expected values of these outcomes for this analysis, I constructed a model that simulates an experimental monitoring program for groundwaterfed side channels utilized by coho and chum salmon. In the simulated monitoring programs, managers choose the number of systems that are monitored, the duration of the monitoring program, and the sampling methods used. For each system and year of the monitoring program, the model simulates the sampling and estimation of species-specific and life-stage-specific survival rates in the side channel and adjacent wild habitat. Tables 
7 through 10 summarize quantities that are estimated, as well as their associated measurement errors for each set of sampling methods (high-precision and low-precision) (see Appendix for more details). The model then uses the data obtained from the simulated monitoring program to estimate the net present value of side channels (which includes only economic costs and benefits) and choose whether to apply this method of enhancement more widely (i.e. to other stocks). In the following sections, I describe in more detail the model used to evaluate different monitoring programs.

\section{Overview of the Model}

The decision analysis model (Figure 10) evaluates each experimental design in terms of its expected net present value (ENPV). Benefits include the economic value of incremental catches of chum and coho salmon resulting from enhancement in the postexperimental period (Figure 1 1). I included the economic benefits of incremental catch of chum salmon as well as coho salmon because chum salmon may also use groundwaterfed side channels for spawning (Sheng et al. 1990) where the two species overlap, such as streams on southeast Vancouver Island. Costs include the costs of monitoring enhanced systems during the experimental period and the costs of building additional side channels in the post-experimental period if the monitoring program concludes that they are worthwhile. I compared different monitoring options with a non-experimental option in which I assumed that managers apply enhancement more widely without first monitoring to determine whether it is worthwhile ("enhancement without monitoring" option). I then calculated the expected net economic benefits of monitoring as the difference between the ENPV of each monitoring option and the ENPV of the 
Table 7. Methods of sampling and assumed coefficients of variation $(\sigma / \mu)$ in abundance estimates for high-precision monitoring program. See Appendix for derivation of coefficients of variation.

Quantity Estimated

Spawner Abundance

Sampling Method

Optimistic

Pessimistic

Chum salmon fry abundance

Sampling Method

Optimistic

Pessimistic

Coho salmon fry abundance

Sampling Method

Optimistic

Pessimistic

Coho salmon smolt abundance

Sampling Method

Optimistic

Pessimistic
Method of sampling used and $\mathrm{CV}$ by location

Side Channel

Wild Habitat

Fence count

Partial Fence plus markrecapture

0

0.1

0

0.2

Fence count

0

Trap efficiency

0.1

0.2

0

Fence count

0

Mark-recapture

0.1

0.25
Fence count

0

0
Trap efficiency

0.1

0.2 
Table 8. Methods of sampling and assumed coefficients of variation $(\sigma / \mu)$ in abundance estimates for low-precision monitoring program. See Appendix for derivation of coefficients of variation.

Quantity Estimated

\section{Spawner Abundance}

Sampling Method

Optimistic

Pessimistic

Chum salmon fry abundance

Sampling Method

Optimistic

Pessimistic

Coho salmon fry abundance

Sampling Method

Optimistic

Pessimistic

Coho salmon smolt abundance

Sampling Method

Optimistic

Pessimistic
Method of sampling used and CV by location

Side Channel

Wild Habitat

Visual Estimates

Visual Estimates

0.1

0.25

0.1

0.4

Fence count

0

0

Hydraulic $x$ Spawners

$\mathrm{n} / \mathbf{a}$

$\mathrm{n} / \mathbf{a}$

Fence count

Hydraulic $x$ Spawners

0

0.81

0.90
Fence count

0

0
Trap efficiency

0.1

0.2 
Table 9. Measurement error variance for effect sizes with high-precision sampling methods.

$$
\text { Variance (v) Standard deviation (v) }
$$

Coho egg-to-fry survival

$\begin{array}{lll}\text { Optimistic } & 0.0199 & 0.1411 \\ \text { Pessimistic } & 0.0998 & 0.3160\end{array}$

Coho fry-to-smolt survival

$\begin{array}{lll}\text { Optimistic } & 0.0199 & 0.1411 \\ \text { Pessimistic } & 0.0998 & 0.3160\end{array}$

Chum egg-to-fry survival

$\begin{array}{lll}\text { Optimistic } & 0.0199 & 0.1411 \\ \text { Pessimistic } & 0.0784 & 0.2801\end{array}$

Table 10. Measurement error variance for effect sizes with low-precision sampling methods.

Variance (v) Standard deviation (v)

Coho egg-to-fry survival

$\begin{array}{lll}\text { Optimistic } & 0.8949 & 0.9460 \\ \text { Pessimistic } & 0.8949 & 0.9460\end{array}$

Coho fry-to-smolt survival

$\begin{array}{lll}\text { Optimistic } & 0.5180 & 0.7197 \\ \text { Pessimistic } & 0.6351 & 0.7969\end{array}$

Chum egg-to-fry survival

$\begin{array}{lll}\text { Optimistic } & 0.8949 & 0.9460 \\ \text { Pessimistic } & 0.8949 & 0.9460\end{array}$


Figure 10. Flow chart for the decision analysis model used to compare experimental monitoring programs for groundwater-fed side channels.

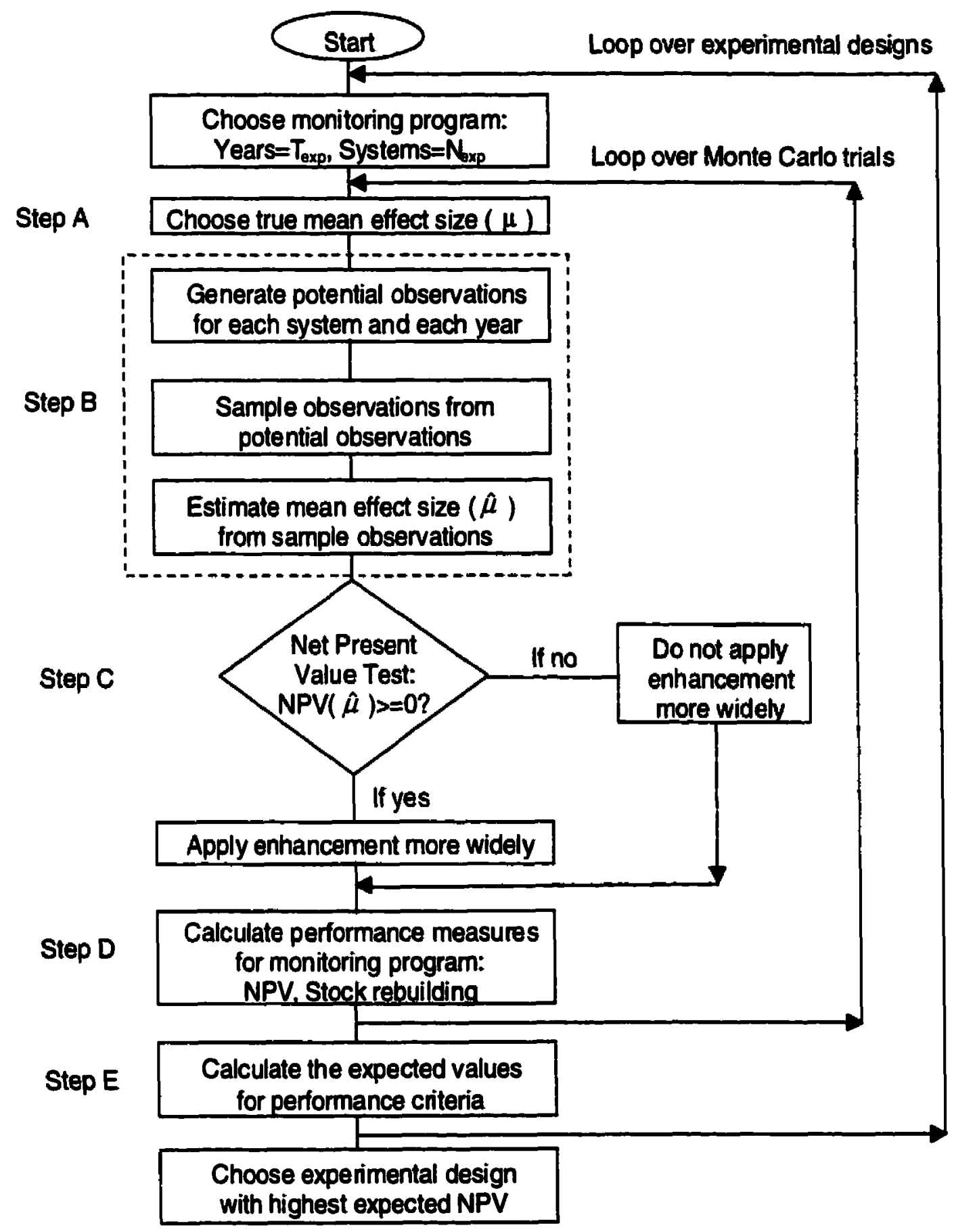


Figure 11. Timeline indicating the sequence of events in an experimental monitoring program and the timing of costs and benefits.

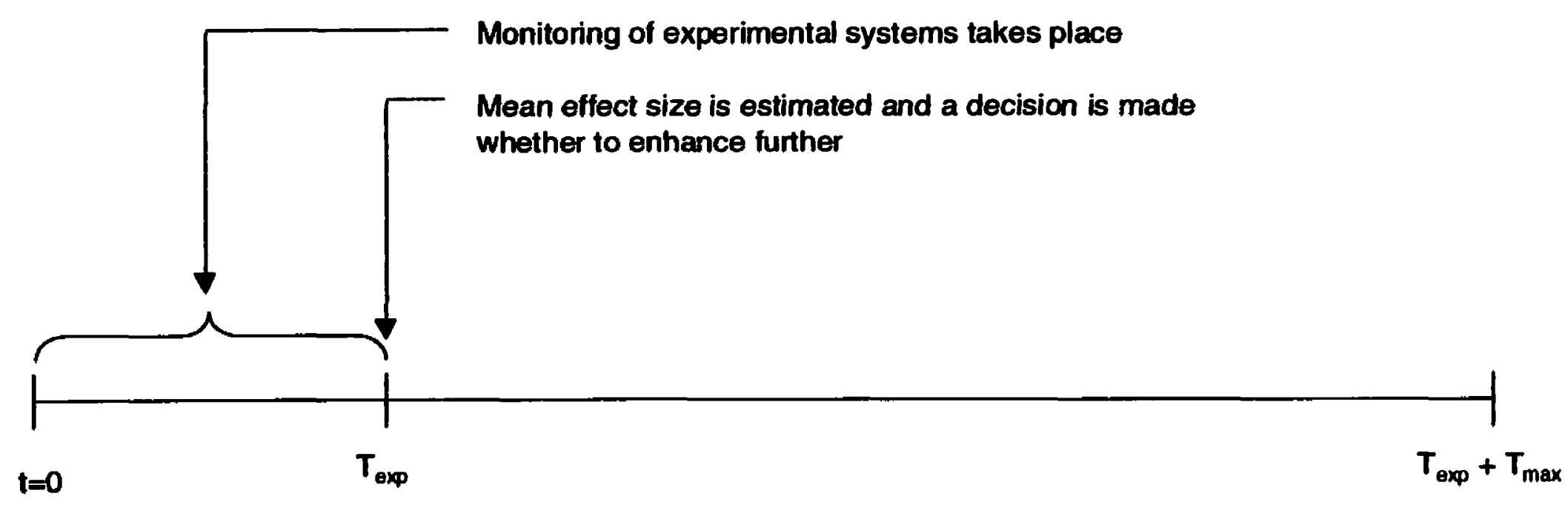

\section{Experimental Costa/Benefits}

Costs = Monitoring costs for $N_{\exp }$ systems over $T_{\text {exp }}$ years.

Benelits $=0$

\section{Post-Experimental Costs/Eenefits}

If $\operatorname{NPV}(\hat{\mu})>=0$ then enhancement applied and,

Costs = Capital costs of $\mathrm{N}_{\text {tol }}$ projects

Benefits = Fish production benefits of $N_{\text {tot }}$ projects from year $T_{\text {exp }}$ to $T_{\text {exp }}+T_{\max }$ given the true state of nature $(\mu)$

If $\operatorname{NPV}(\hat{\mu})<0$ then enhancement not applied and,

Costs $=$ Benefits $=0$ 
"enhancement without monitoring" option. In addition, the model calculates purely biological measures of the rate of stock rebuilding to higher abundances. However, I did not include such conservation benefits in my benefit-cost calculations because there is no agreement within management agencies regarding the monetary value for rebuilding depressed coho stocks. However, this framework could incorporate such values once they have been articulated.

For each Monte Carlo trial, the model randomly selects the true mean effect size $(\mu)$ for each life-stage and species from specified distributions. A potential pool of observations for the observed effect size is generated and a sample is drawn from this pool based on the experimental design (i.e. duration of monitoring program, number of monitored systems, and sampling methods for estimating survival rates for wild and enhanced fish at the affected life-stages). The mean effect sizes for each species and lifestage are then estimated from the sample of observed effect sizes. The net present value of enhancement is calculated based on estimated mean effect sizes. If the estimated net present value (NPV $(\boldsymbol{A}))$ is greater than or equal to zero, it is assumed that managers will apply enhancement to all non-experimental systems in the post-experimental period and, if not, then enhancement is not applied more widely. The net present value of the monitoring program is then calculated based on the true net present value of each enhancement project (NPV $(\mu)$ ) and the subsequent decision (i.e. whether to enhance or not to enhance). The expected net present value is calculated by averaging the net present value of the monitoring program over 2,000 Monte Carlo trials. The performance of each management action is also evaluated in terms of biological performance measures (such as the expected number of coho salmon recruits in a given year). 


\section{Model Details}

In the preceding sections, I described a general decision analysis framework in simple terms, where it was assumed that there is only one uncertain biological effect. However, in the case of groundwater-fed side channels utilized by coho and chum salmon, I consider three uncertain biological effects. These are the multiplicative increases in coho egg-to-fry, coho fry-to-smolt, and chum egg-to-fry survival rates.

I assume enhanced survival rates for each life-stage and species are related to corresponding wild survival rates by the following equation:

$$
\left(\begin{array}{c}
\text { Observed Enhanced } \\
\text { Survival Rate }
\end{array}\right)=\left(\begin{array}{c}
\text { Observed Wild } \\
\text { Survival Rate }
\end{array}\right) \cdot \exp \left(\hat{\theta}_{i \xi}\right)
$$

where $\exp \left(\hat{\theta}_{i t}\right)$ is the multiplicative increase in the survival rate of enhanced fish observed in system $i$ and year $t$. Because survival rates for Pacific salmon are log-normally distributed (Bradford, 1995), I consider the difference in the natural logarithms of observed enhanced and wild survival rates to be an appropriate measure of effect size:

$$
\hat{\theta}_{i t}=\ln \left(\begin{array}{c}
\text { Observed Enhanced } \\
\text { Survival Rate }
\end{array}\right)-\ln \left(\begin{array}{c}
\text { Observed Wild } \\
\text { Survival Rate }
\end{array}\right)
$$

For each species and life-stage, the observed effect size has 5 components: ( $I$ ) the true mean effect size $(\mu),(2)$ system-specific effects $\left(X_{i}\right),(3)$ year-specific effects $\left(Y_{t}\right),(4)$ natural variability or process error $\left(e_{i i}\right)$, and measurement error $\left(v_{i t}\right)$ (see equation (14)). In the decision analysis, I explicitly considered these sources of uncertainty in the true mean effect size for coho egg-to-fry, coho fry-to-smolt, and chum egg-to-fry survival rates. Headings below correspond with steps shown in Figure 10. 


\section{Step A. True mean effect sizes}

The probability distributions for the true mean effect sizes reflect the prior beliefs managers have about the possible values of the true mean effect size for each life-stage and species. The true mean effect size for a given species and life-stage $(\mu)$ relates true mean survival rates for wild and enhanced fish by the following equation:

$$
\left(\begin{array}{c}
\text { Mean wild } \\
\text { survival rate }
\end{array}\right) \cdot \exp (\mu)=\left(\begin{array}{c}
\text { Mean enhanced } \\
\text { surviva! rate }
\end{array}\right)
$$

Hence, the mean proportional increase in the survival rate is given by $\exp (\mu)-1$. I was unable to obtain sufficient data to derive clear prior probability distributions for the mean proportional increases in survival rates, hence I assumed these were uniformly distributed. It should be noted that this is not equivalent to assuming that the true mean effect size $(\mu)$ is uniformly distributed, because the relationship between $\mu$ and the mean proportional increase in survival is non-linear.

Using uniform distributions, six parameters (the minimum and maximum mean proportional increases in survival rates for each life-stage and species) capture the prior beliefs of managers regarding the efficacy of side channels. By selecting the true mean proportional increase in survival rate from uniform probability distributions, the model assumes that values outside of the range between the minimum and maximum have a zero probability and values within this range have an equal probability. I assumed minimum and maximum values for the mean proportional increases in coho and chum egg-to-fry survival rates of 0 and 1.0 , which correspond to minimum and maximum values for $\mu_{1}$ and $\mu_{3}$ of 0 and 0.693 (Table 11). For coho fry-to-smolt survival, I assumed 
minimum and maximum proportional increases of 0.5 and 1.5 respectively, which correspond to minimum and maximum values for $\mu_{2}$ of 0.405 and 0.916 .

\section{Step B. Observed effects and estimated mean effect sizes}

The observed effect size in any given year and system for the three measures of effect size are drawn from the following equations:

(20) $\hat{\theta}_{1, i t}=\mu_{1}+X_{1, i}+Y_{1, t}+e_{1, i t}+v_{1, i t}$

(21) $\hat{\theta}_{2, i t}=\mu_{2}+X_{2, i}+Y_{2, s}+e_{2, i t}+v_{2, i t}$

(22) $\quad \hat{\theta}_{3, i}=\mu_{3}+X_{3, i}+Y_{3, t}+e_{3, i t}+v_{3, i t}$

where $\hat{\theta}_{1, i t}, \hat{\theta}_{2, i t}$, and $\hat{\theta}_{3, i t}$ are the observed differences in the natural logarithms of enhanced and wild in coho egg-to-fry, coho fry-to-smolt, and chum egg-to-fry survival rates in system $i$ in year $t$. Values for $\mu_{1}, \mu_{2}$, and $\mu_{3}$ are the true mean effect sizes for each life-stage and species. The remaining terms in each equation represent the sources of variation in observed effect sizes. The $X$ 's represent system-specific effects (shared across years), the $Y$ 's represent year-specific effects (shared across systems), the $e$ 's represent natural variation (i.e. process error) in effect size and the $v$ 's represent measurement error. I assumed that system-specific effects, year-specific effects, process errors, and measurement ertors all have a mean of zero, hence, the variation in observed effect sizes is characterized by the variance for each source of variation and each measure of effect size.

True mean effect sizes $\left(\mu_{1}, \mu_{2}, \mu_{3}\right)$ for each Monte Carlo trial are selected as described in Step A. However, the components of variation that lead to estimated effect 
sizes (i.e. system-specific effects, year-specific effects, process errors, and measurement errors) are randomly selected from normal probability distributions with standard deviations shown in Tables 9 through 11.

In the baseline simulations, I assumed that monitoring programs would utilize relatively "high-precision" sampling methods (i.e. methods with low measurement error) to estimate abundance and survival rates of enhanced and wild fish at various life-stages, and, hence, mean effect sizes of enhancement. I assumed that per-system per-year monitoring costs of $\$ 80,000$ with monitoring programs using these "high-precision" sampling methods (Michael Bradford, pers. comm.). More details on the sampling methods used and associated measurement error variances in abundance estimates and, hence, effect sizes, are provided in Tables 7 through 10.

I derived estimates for process error and measurement error variances in wild and enhanced coho egg-to-fry, coho fry-to-smolt and chum egg-to-fry survival rates using data on survival rates for coho and chum salmon in the wild and in side channels and studies on the precision of measuring fish abundance using various sampling methods (see Appendix for details). I then used these estimates for variances in life-stage and speciesspecific survival rates to derive estimates for the measurement error and process error variances in observed effect sizes in equations (20) to (22) (see Appendix for details).

I assumed that the standard deviation for system-specific effects in equations (20) to (22) was 0.25 , which corresponds to a variance of 0.0625 . In other words, while I assumed that there were differences among channels in observed incremental increases in survival rates due to enhancement, the variability in these system-specific effects was moderate. I assumed that the standard deviation, and hence variance, of year-specific 
Table 11. Baseline parameter values for decision analysis on alternative monitoring programs for groundwater-fed side channels.

Parameter

Minimum mean proportional increase

Maximum mean proportional increase

Standard deviation for system-specific Effects

Standard deviation for year-specific Effects

Standard deviation for process error

Standard deviation for measurement error

\section{Measure of Effect Size}

Coho egg- Coho fry-to- Chum eggto-firy smolt to-fry survival rate survival rate survival rate

Per-system per-year monitoring cost (high-precision sampling) $\$ 80,000$ Number of systems to which monitoring results are applied $\left(\mathbf{N}_{\mathrm{tot}}\right)$ Real discount rate 
effects was zero, which when combined with the assumed mean of zero, reflects the assumption that year-to-year variation in effect size for each enhanced system (or stock) responds independently of that of other enhanced systems. Bradford (1999) found that coho smolt abundances were generally uncorrelated for streams more that $20-30 \mathrm{~km}$ apart, which supports the assumption of effects across systems within a given year.

The estimated mean effect sizes are calculated by averaging over all observed values of effect sizes. That is:

$$
\hat{\mu}_{1}=\frac{1}{N_{\exp } T_{\exp }} \sum_{i=1}^{N_{a p}} \sum_{t=1}^{T_{\text {ap }}} \hat{\theta}_{\mathrm{l}, i t}
$$

$$
\hat{\mu}_{2}=\frac{1}{N_{\exp } T_{\exp }} \sum_{i=1}^{N_{\text {ap }}} \sum_{t=1}^{T_{\text {ap }}} \hat{\theta}_{2, i t}
$$

$$
\hat{\mu}_{3}=\frac{1}{N_{\exp } T_{\exp }} \sum_{i=1}^{N_{a p p}} \sum_{t=1}^{p_{a p}} \hat{\theta}_{3, i t}
$$

where $N_{\text {exp }}$ is the number of systems monitored and $T_{\text {exp }}$ is the duration of the monitoring program in years.

\section{Step C. Net Present Value of a Side Channel}

At the conclusion of the experimental monitoring program, an estimate of the net present value of a side channel is calculated based on the estimated mean effect sizes (see equations (23) to (25)). If the estimated NPV of a side channel is greater than or equal to zero, it is assumed that enhancement is applied more widely, while enhancement is not applied further if the NPV of a side channel is less than zero. With the "perfect information" scenario, it is assumed that managers know the true mean effect sizes with 
certainty and, hence, apply enhancement if and only if the true NPV of a side channel is greater than or equal to zero, while it is assumed that enhancement is always applied in the "enhancement without monitoring" option.

The net present value (NPV) of a side channel is calculated by using the following equation:

$$
N P V=P V_{\text {Coho }}\left(\mu_{1}, \mu_{2}\right)+P V_{\text {Chum }}\left(\mu_{3}\right)-P V C
$$

where $P V_{\text {Cuhu }}\left(\mu_{1}, \mu_{2}\right)$ is the present value of coho benefits, $P V_{C h u m}\left(\mu_{3}\right)$ is the present value of chum benefits and PVC is the present value of costs of a side channel. Details for the calculation of each of these components of the net present value of a side channel are described in more detail below.

\section{Present Value of Incremental Coho Catch}

The value of incremental coho catch is calculated by simulating the dynamics of the coho population both with and without enhancement. The present value of the catch without enhancement is then deducted from the present value of the catch with enhancement to provide the value of incremental coho catch.

Coho population dynamics with the side channel are calculated using the biological model described in section II. When calculating the estimated NPV of enhancement (step $C$ ), the estimated effect size in the coho egg-to-fry survival rate $\left(\hat{\mu}_{1}\right)$ is used in place of $\theta_{1}$ in equation (6) and the estimated mean effect size in the densityindependent coho fry-to-smolt survival rate $\left(\hat{\mu}_{2}\right)$ is used in place of $\theta_{2}$ in equation (11). When calculating the true NPV of enhancement, where enhancement is applied more 
widely (step C), the true mean effect sizes are substituted into equations (6) and (11). Values for other biological and economic parameters used in the net present value calculations are shown in Table 1.

Recruitment and catch are modelled using the following equations:

$$
\text { Recruits }=\text { Smolts } \bullet \text { Marine }
$$

where Marine is the marine survival rate (i.e. smolt to recruit) for coho.

$$
\text { Spawners }=\text { Recruits }- \text { Catch }
$$

In both cases with and without enhancement, I assumed that a fixed escapement policy is employed, in which the target escapement is set to be the minimum number of spawners required for the population to be fully-seeded. Only recruits in excess of this target are harvested. That is:

$$
\text { Catch }=\max \{\text { Recruits }- \text { Target }, 0\}
$$

where Target is the target escapement.

The freshwater life-stages for coho without enhancement are simulated with the following equations, while freshwater life-stages with enhancement are simulated using the biological model described in section $\mathrm{II}$.

(30) Females $=$ Spawners $\bullet P f$

where $P f$ is the proportion of female spawners.

$$
\text { Fry }=\text { Females } \bullet \text { Fecundity } \bullet a
$$

where Fecundity is the number of eggs per female and $a$ is the egg-to-fry survival rate for wild coho eggs.

$$
\text { Smolts }=\min \left\{\text { Fry } \bullet \text {, MaxSmolts } s_{W} \bullet \text { WildArea }\right\}
$$


where $b$ is the density-independent fry-to-smolt survival rate for wild coho, MaxSmoltsw is the maximum number of smolts produced per $\mathrm{m}^{2}$ of wild habitat and WildArea is the total area of wild habitat in $\mathrm{m}^{2}$.

The value of catch each year both with and without enhancement is calculated as follows:

$$
V_{t}=\operatorname{Catch}_{t} \bullet\left[\text { Prop Spon }_{\text {Sport }} \bullet\left(1-B_{\text {Srop }}{ }_{\text {Spon }}\right) \bullet B_{\text {com }} \bullet \text { Weight }\right]
$$

where $V_{t}$ is the value of catch in year $t$, Catch $_{t}$ is catch in numbers of fish in year $t$,

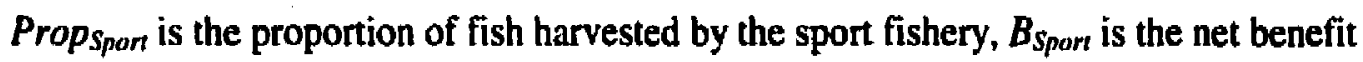
(per fish) to the sport fishery, $\boldsymbol{B}_{C u m}$ is the net benefit (per $\mathrm{kg}$ fish) to the commercial fishery, and Weight is the average weight of an adult coho (in $\mathrm{kg}$ ).

I have assumed that 50 percent of the catch would go to the sport fishery and the remaining 50 percent would go to the commercial fishery. The net benefit per fish caught in the sport fishery is $\$ 19.66$ and the net benefit per kilogram of fish caught in the commercial fishery is $\$ 3.05$. These values were taken from the Salmonid Enhancement Frogram cost-benefit analysis model (Chris Sporer, DFO, pers. comm.) and translated into 1998 dollars. I have assumed an average weight per adult coho of $3.5 \mathrm{~kg}$.

The present value of incremental coho benefits tesulting from enhancement is calculated as:

$$
P V_{\text {Coho }}\left(\mu_{1}, \mu_{2}\right)=\sum_{i=1}^{T_{\max }}\left(\frac{1}{1+\text { disc }}\right)^{t} \cdot\left(V_{E \text { Enhances }}-V_{\text {NaEnhances }}\right)
$$

where Tmax is the last year in which benefits of the side channel are realized, disc is the real discount rate, $V_{\text {Enhances, }}$ is the value of coho catch in year $t$ with enhancement, and $V_{\text {NoEnnunce, }}$ is the value of coho catch in year $t$ without enhancement. 
I have assumed that each side channel will effectively provide benefits to juvenile coho for 20 years, but since the last set of coho juveniles will not return as adults until 3 years after their parents spawn, Tmax was set to 23 years. For the baseline simulations, I assumed a real discount rate of 5 percent.

\section{Present Value of Incremental Chum Catch}

I aiso incorporated the benefits of incremental catches of chum salmon from groundwater-fed side channels. As is the case with coho salmon, groundwater-fed side channels may provide a more stable incubation environment for chum salmon eggs, hence increasing their freshwater survival rate and the resulting adult recruits (Bonnell, 1991).

The number of chum fry produced from a side channel is modelled using the following equation:

$$
F r y_{E}=\left(F r y / m^{2}\right) \bullet \text { SideArea }
$$

where $F r y / m^{2}$ is the number of chum fry produced per $\mathrm{m}^{2}$ of side channel and SideArea is the area of the side channel in $\mathrm{m}^{2}$. Bonnell (199l) reports an average value of 297 $(\mathrm{N}=30)$ chum fry per $\mathrm{m}^{2}$ from groundwater-fed side channels designed for chum salmon in British Columbia. This figure may provide an overestimate of the number of chum fry produced from side channels designed for use by coho salmon, because coho juveniles have been observed to feed on chum fry in side channels (Sheng et al., 1990). Hence, I have assumed a value of 200 chum fry per $\mathrm{m}^{2}$ of side channel.

The number of fry that would have been produced if the eggs laid in the side channel had been laid in the wild can be expressed in terms of the relative egg-to-fry survival rates in the side channel and in the wild: 


$$
F r y_{W}=F r y_{E} \cdot\left[\left(\frac{F r y}{E g g}\right)_{W} /\left(\frac{F r y}{E g g}\right)_{E}\right]=F r y_{E} \cdot\left(\frac{1}{\exp \left(\mu_{3}\right)}\right)
$$

where $F r y_{E}$ is the number of fry produced if all eggs are laid in the side channel, $(F r y / E g g)_{W}$ is the wild egg-to-fry survival rate for chum, $(F r y / E g g)_{E}$ is the enhanced eggto-fry survival rate and $\mu_{3}$ is the mean effect size in the chum egg-to-fry survival rate for enhanced chum salmon.

Incremental fry production is the number of fry produced if all eggs had been laid in the side channel less the fry produced if all eggs had been laid in the wild:

$$
F r y_{\text {tncremenul }}=F r y_{E} \cdot\left(1-\frac{1}{\exp \left(\mu_{3}\right)}\right)=F r y_{E} \cdot\left(\frac{\exp \left(\mu_{3}\right)-1}{\exp \left(\mu_{3}\right)}\right)
$$

Incremental recruits of adult salmon are calculated as:

$$
\text { Recruits }_{\text {Incrementul }}=F r y_{\text {Incremenul }} \bullet \text { Marine }
$$

where Marine is the marine (fry-to-recruit) survival rate for chum salmon. Koning and Keeley (1997) suggest using a marine survival rate of 0.7 percent for evaluating benefits to chum salmon from habitat enhancement projects.

The annual value of incremental chum salmon benefits are calculated as follows:

$$
V_{t}=0 \quad \text { for } t<4
$$

$$
V_{t}=\text { Recruits }_{\text {Incremental }} \bullet B_{\text {Chum }} \bullet \text { Weight } \quad \text { for } t \geq 4
$$

No incremental chum are harvested for the first three years after the side channel is constructed, because it takes three years before the first chum benefiting from enhancement return as adults to the fishery. $B_{C h u m}$ is the net benefit to the commercial fishery per $\mathrm{kg}$ of chum harvested and Weight is the weight of chum salmon in $\mathrm{kg}$. I assumed a weight of $3.0 \mathrm{~kg}$ for each chum recruit and a value of $\$ 1.82$ for $B_{C h u m}$, which is 
the value used in the SEP cost-benefit model inflated to 1998 dollars.

Finally, the present value of chum benefits is calculated as:

(41) $\quad P V_{\text {Chum }}\left(\mu_{3}\right)=\sum_{i=1}^{T_{\max }}\left(\frac{1}{1+\text { disc }}\right)^{\prime} \cdot V_{1}$

\section{Present Value of Side Channel Costs}

Only capital costs have been included in the present value of side channel costs. Hence, the present value of costs per side channel are simply:

$$
\text { PVC }=\left(\text { CapitalCost } / \mathrm{m}^{2}\right) \bullet \text { SideArea }
$$

Mean capital costs per $\mathrm{m}^{2}$ of side channel (in 1987 dollars) for chum salmon were $\$ 24.56$ for channels using existing gravel and $\$ 28.99$ for channels using graded gravel (Bonnell, 1991). Hence, I assumed a value of $\$ 27.00$ per $\mathrm{m}^{2}$ in 1987 dollars. This value was inflated into 1998 dollars using the Canadian Consumer Price Index for all goods, yielding a value of $\$ 35.98$ per $\mathrm{m}^{2}$ of side channel.

\section{Step D. Performance Measures for Management Options}

Once a decision is made whether to apply enhancement more widely (step C), the model simulates outcomes based on the decision and the true mean effect sizes. The model determines whether a correct decision has been made, simulates the dynamics of coho and chum populations, and calculates the net present value for different management alternatives. It should be noted that the NPV of each management option (i.e. different monitoring options, "enhancement without monitoring" option, and the "perfect information" scenario) is not equivalent to the NPV of a side channel. The latter 
is only one component of the NPV for the different management options, as I illustrate in the following sections.

\section{Net Present Value of Monitoring Program}

The net present value of each monitoring program includes the costs of monitoring and the net benefits of the subsequent decision following the conclusion of the monitoring program (Figure 11). During the experimental period, monitoring costs are incurred. The present value of monitoring costs is calculated as:

$$
P V_{m o n_{-} c o s t}=\sum_{i=1}^{t_{\text {ap }}} N_{\text {exp }} \bullet \operatorname{Cost}_{\text {mon }} \bullet\left(\frac{1}{1+d i s c}\right)^{l}
$$

where $T_{\exp }$ is the duration of the monitoring program, $N_{\exp }$ is the number of systems monitored and Cost $_{\text {mun }}$ is the cost per system per year of monitoring. Costs and benefits of enhancement are ignored in the experimental period under the assumption that monitoring takes place in pre-existing side channels, or side channels that would have been constructed anyway, with or without the monitoring program.

In the post-experimental period, the costs and benefits of the monitoring program are dependent upon the decision made at the conclusion of the monitoring program (i.e. whether to enhance more widely). If the estimated NPV of a side channel given the estimated effect sizes for each life-stage and species $\left(\operatorname{NPV}\left(\hat{\boldsymbol{\mu}}_{1}, \hat{\boldsymbol{\mu}}_{2}, \hat{\boldsymbol{\mu}}_{3}\right)\right)$ is greater than or equal to zero, then it is assumed that enhancement is applied more widely, and the net present value of the decision is:

$$
N P V_{\text {decision }}=N_{\text {tot }} \bullet N P V\left(\mu_{1}, \mu_{2}, \mu_{3}\right) \cdot\left(\frac{1}{1+d i s c}\right)^{T_{a p}}
$$

where $N_{\text {tor }}$ is the total number of systems to which enhancement is applied and 
$N P V\left(\mu_{1}, \mu_{2}, \mu_{3}\right)$ is the true net present value of each side channel. It should be noted that any benefits and costs of enhancement after the conclusion of the monitoring program are discounted to take account of the duration of the monitoring program. If the estimated NPV of a side channel $\left(\operatorname{NPV}\left(\hat{\mu}_{1}, \hat{\mu}_{2}, \hat{\mu}_{3}\right)\right)$ is less than zero, then it is assumed that enhancement is not applied to other systems and the present value of the subsequent decision is zero.

The net present value of the monitoring program is the net present value of the subsequent decision about applying enhancement more widely less the present value of monitoring costs. Hence:

$$
\begin{array}{ll}
N P V_{\text {monituring }}=N P V_{\text {decision }}-P V_{\text {mon_cuart }} \text { if } N P V\left(\hat{\mu}_{1}, \hat{\mu}_{2}, \hat{\mu}_{3}\right) \geq 0 \\
N P V_{\text {monituring }}=-P V_{\text {men_cust }} \quad \text { if } N P V\left(\hat{\mu}_{1}, \hat{\mu}_{2}, \hat{\mu}_{3}\right)<0
\end{array}
$$

Net Present Value of "Perfect Information"

For comparison with management options, I also calculated a case in which it is assumed that managers know the true mean effect sizes with certainty (i.e. they have "perfect information"). In this case, I assume that they apply enhancement to all $N_{t u t}$ systems if the true net present value of enhancement is greater than or equal to zero and do not apply enhancement if the true net present value of enhancement is less than zero. Hence, the net present value of "perfect information" is:

$$
\begin{array}{ll}
N P V_{\text {perfect_info }}=N_{\text {tut }} \infty N P V\left(\mu_{1}, \mu_{2}, \mu_{3}\right) & \text { if } N P V\left(\mu_{1}, \mu_{2}, \mu_{3}\right) \geq 0 \\
N P V_{\text {perfect_info }}=0 & \text { if } N P V\left(\mu_{1}, \mu_{2}, \mu_{3}\right)<0
\end{array}
$$


Net Present Value of "Enhancement without Monitoring"

I also calculated the net present value under the assumption that managers apply enhancement immediately without monitoring any side channels. The net present value of this option is:

$$
N P V_{n u_{\text {_monitoring }}}=N_{\text {tut }} \bullet N P V\left(\mu_{1}, \mu_{2}, \mu_{3}\right)
$$

\section{Step E. Expected Value of Performance Measures}

To assess the efficacy of different monitoring programs and other management options (e.g. "perfect information" and "enhancement without monitoring"), the model calculates several performance measures to summarize outcomes over all Monte Carlo trials. These are as follows:

1. Probability of a correct decision - A correct decision is made when either: (1) enhancement is applied and $N P V\left(\mu_{1}, \mu_{2}, \mu_{3}\right) \geq 0$ or (2) enhancement is not applied and $N P V\left(\mu_{1}, \mu_{2}, \mu_{3}\right)<0$. The probability of a correct decision is simply the proportion of Monte Carlo trials in which a correct decision is made.

2. Expected Net Present Value (ENPV) - This was calculated by averaging the net present value of the given policy option over all Monte Carlo trials.

3. Expected Number of Coho Recruits - I also calculated the expected number of recruits of coho salmon in each population in years 7, 13, and 22 in the post-experimental period. These years correspond to 2,4 , and 7 generations after a decision is made regarding whether to apply enhancement to the non-experimental systems.

4. Probability that coho recruits exceed a given target - Finally, numbers of coho 
recruits in years 7, 13, and 22 in the post-experimental period (i.e. 2, 4, and 7 generations after a decision is made whether to apply enhancement more widely) were compared to arbitrarily set recruitment targets and the probabilities of recruitment exceeding these targets (among the Monte Carlo trials) were calculated for each management option. I calculated recruitment targets as a percentage of the maximum number of recruits that could return to the population without enhancement. Under the baseline assumptions, initial coho spawner abundance is approximately 10 percent of this value, while recruitment targets in years 7,13 , and 22 are 50 percent, 75 percent, and 100 percent of maximum wild recruitment, respectively.

I evaluated different monitoring options in terms of their performance relative to the "enhancement without monitoring" option. The expected net economic benefits of monitoring are simply the difference between the ENPV of a given monitoring option and the ENPV of the "enhancement without monitoring" option. I expressed the net benefits of monitoring in these terms, because "enhancement without monitoring" is the most likely management option in the absence of experimental management approaches.

\section{Sensitivity Analysis}

To illustrate the most important components of the net present value of a side channel (not the ENPV of monitoring programs and other management options), I performed sensitivity analyses on the net present value with respect to: (1) the mean proportional incrense in the survival rate for each species and life-stage, (2) the marine survival rate for coho, and (3) the real discount rate.

As a basis for comparison, I conducted the full decision analysis for monitoring 
programs using two different sets of sampling methods: (l) "high-precision" sampling methods (the baseline case), (2) "low-precision" sampling methods. It should be noted, however, that the measurement error associated with different sampling methods is very uncertain, hence, I derived "optimistic" and "pessimistic" sets of estimates for the variances of measurement error for each set of sampling methods (see Appendix for details).

In the baseline case, estimates for process error variance in survival rates were derived by subtracting "reasonable" values of measurement error variance from estimates of total variance in survival rates from Bradford (1995). This had the effect of reducing the values for process error variances. However, given the lack of information on specific sampling methods and sampling intensity used in the studies reviewed by Bradford (1995), I repeated the analysis under the pessimistic assumption that all of the interannual variation in estimated survival rates was due to process error (i.e. that is, I assumed that there was no measurement error in Bradford's (1995) variance estimates (see Appendix for details).

Additional sensitivity analyses were conducted on the prior probabilities for mean effect sizes, the number of systems to which experimental results are applied, the standard deviation of system-specific effects, the standard deviation of year-specific effects, and the real discount rate. Table 12 provides a description of all the decision analysis scenarios, baseline as well as sensitivity analyses. In all cases, parameters were held at their "baseline" values unless stated otherwise. 
Table 12. Summary of parameter values for sensitivity analyses performed. Parameter values and assumptions in each case are the same as the baseline case except where stated otherwise.

\section{Scenario}

\section{Description}

Precision of sampling method and magnitude of variance estimates

Baseline

- High-precision sampling

$$
\text { Cost }_{\text {mon }}=\$ 80,000
$$

- Optimistic measurement error estimates

- Low process error estimates

- Standard deviation of process errors

- Standard deviation of measurement error

High-precision sampling with pessimistic measurement error variance estimates

- Standard deviation of measurement error

Low-precision sampling with optimistic measurement error variance estimates

$$
\begin{gathered}
\sigma_{e, 1}=0.281, \sigma_{e, 2}=0.516, \sigma_{e, 3}=0.538 \\
\sigma_{v, 1}=\sigma_{v, 2}=\sigma_{v, 3}=0.141
\end{gathered}
$$

- Standard deviation of measurement error

Low-precision sampling with pessimistic measurement error variance estimates

- Standard deviation of measurement error

$$
\sigma_{v, \mathrm{I}}=0.316, \sigma_{v, 2}=0.316, \sigma_{v, 3}=0.280
$$

$$
\operatorname{Cost}_{\text {men }}=\$ 55,000
$$

$$
\sigma_{v, 1}=0.946, \sigma_{v, 2}=0.719, \sigma_{v, 3}=0.946
$$

$$
\operatorname{Cost}_{\text {mon }}=\$ 55,000
$$

$$
\sigma_{v, 1}=0.946, \sigma_{v, 2}=0.797, \sigma_{v, 3}=0.946
$$

High process error variance estimates

- Standard deviation of process errors $\quad \sigma_{e, 1}=0.376, \sigma_{e, 2}=0.5162, \sigma_{e, 3}=0.593$ 
Scenario

Prior probabilities - Minimum and maximum values for the mean proportional increase in chum egg-to-fry survival rate

Baseline

Alternative scenarios

Number of systems to which monitoring results apply

Baseline

Alternative scenarios

Real discount rate

Low discount rate

Baseline

High discount rate

\section{Standard deviations for system-specific}

effects

No system-specific effects

Baseline - moderate system-specific effects

Large system-specific effects

Standard deviation of year-specific effects

Baseline - no year-specific effects

Moderate year-specific effects

Large year-specific effects
Description

Minimum $=0$, Maximum $=1$

Minimum $=0.25$, Maximum $=1$

Minimum $=0.5$, Maximum $=1$

$$
\begin{gathered}
N_{t o t}=100 \\
N_{t o t}=50,150 \text { and } 200
\end{gathered}
$$

Discount rate $=3 \%$

Discount rate $=5 \%$

Discount rate $=10 \%$

$$
\begin{gathered}
\sigma_{X, 1}=\sigma_{X, 2}=\sigma_{X, 3}=0 \\
\sigma_{X, 1}=\sigma_{X, 2}=\sigma_{X, 3}=0.25 \\
\sigma_{X, 1}=\sigma_{X, 2}=\sigma_{X, 3}=0.5
\end{gathered}
$$

$$
\begin{gathered}
\sigma_{Y, 1}=\sigma_{Y, 2}=\sigma_{Y, 3}=0 \\
\sigma_{Y, 1}=\sigma_{Y, 2}=\sigma_{Y, 3}=0.25 \\
\sigma_{Y, 1}=\sigma_{Y, 2}=\sigma_{Y, 3}=0.5
\end{gathered}
$$




\section{RESULTS AND DISCUSSION}

\section{Net Present Value of a Side Channel}

\section{A. Baseline Results}

I calculated the NPV of a side channel where it is assumed that the mean proportional increases in coho egg-to-fry, coho fry-to-smolt, and chum egg-to-fry survival rates were 0.5 . Under these assumptions, the NPV of a $2,000 \mathrm{~m}^{2}$ side channel is $-\$ 11,952$ and the benefit-cost ratio for a side channel is 0.83 . Thus, if we were to assume that these proportional increases in survival rates were true, enhancement would not be worthwhile from a purely economic perspective, because the capital costs of constructing a side channel outweigh the benefits of incremental catch generated given the assumed magnitude of biological effects of enhancement. However, the mean effect sizes for each life-stage and species affected by side channels are uncertain. It should be noted that the value of incremental coho catch made up only a small proportion of the economic benefits of the side channel ( 8.6 percent). The remaining benefits were derived from incremental chum catch (91.4 percent). This result was due to the low initial spawner abundances for the coho population (because these stocks are assumed to be depressed) and the poor marine survival rate.

\section{B. Sensitivity Analysis}

The net present value of a side channel was equally sensitive to changes in the proportional increases in coho egg-to-fry $\left(\exp \left(\theta_{1}\right)-1\right)$ and coho fry-to-smolt $\left(\exp \left(\theta_{2}\right)-1\right)$ survival rates (Figure 12). However, the NPV was much more sensitive to the proportional increase in the chum egg-to-fry survival rate $\left(\exp \left(\theta_{3}\right)-1\right)$. Hence, from a 
Figure 12. Net present value of a side channel as a function of the proportional increases in the: (A) coho egg-to-fry survival rate, (B) coho fry-to-smolt survival rate, and $(C)$ chum egg-to-fry survival rate.

(A) Coho egg-to-fry survival rate
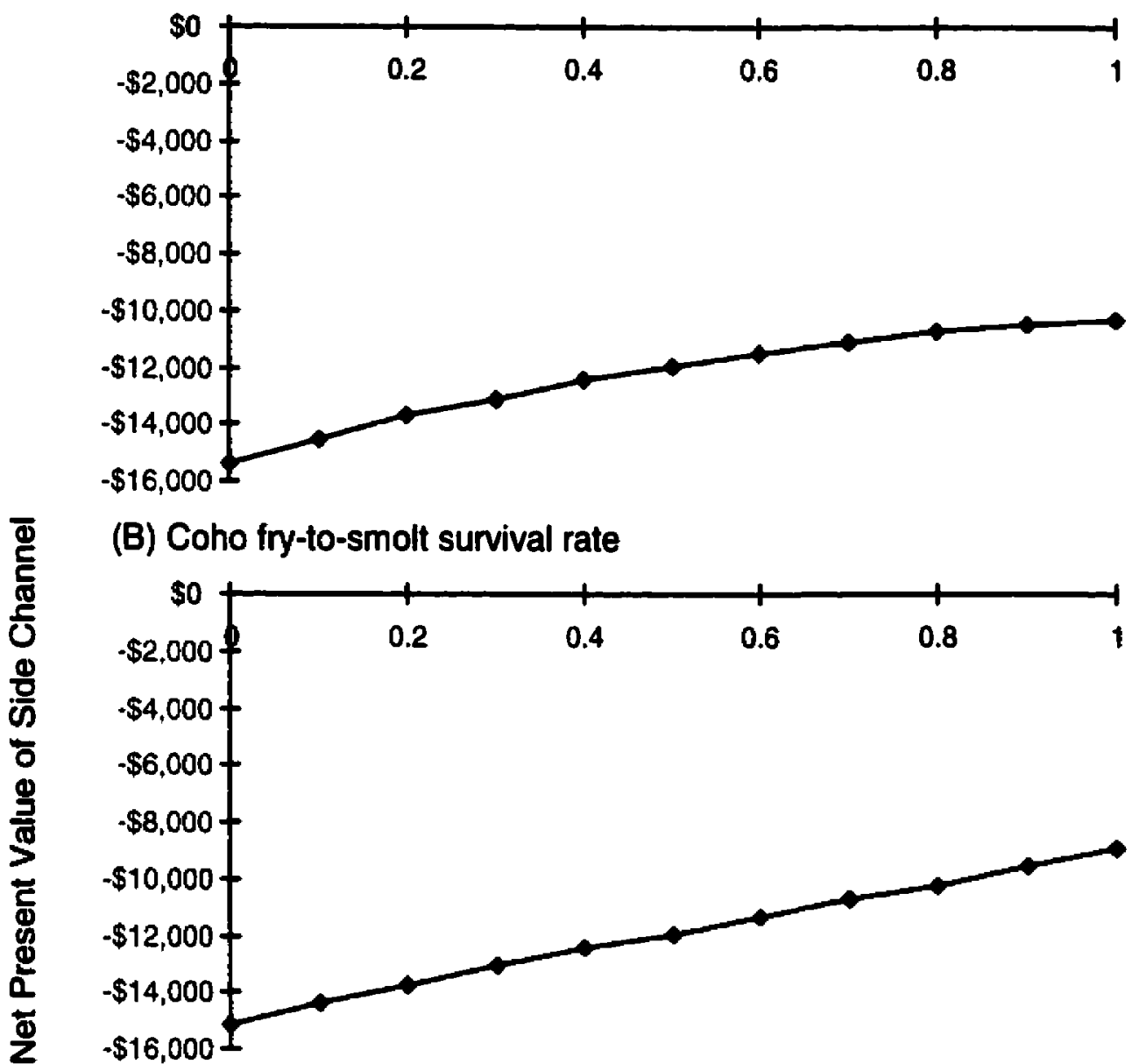

(C) Chum egg-to-fry survival rate

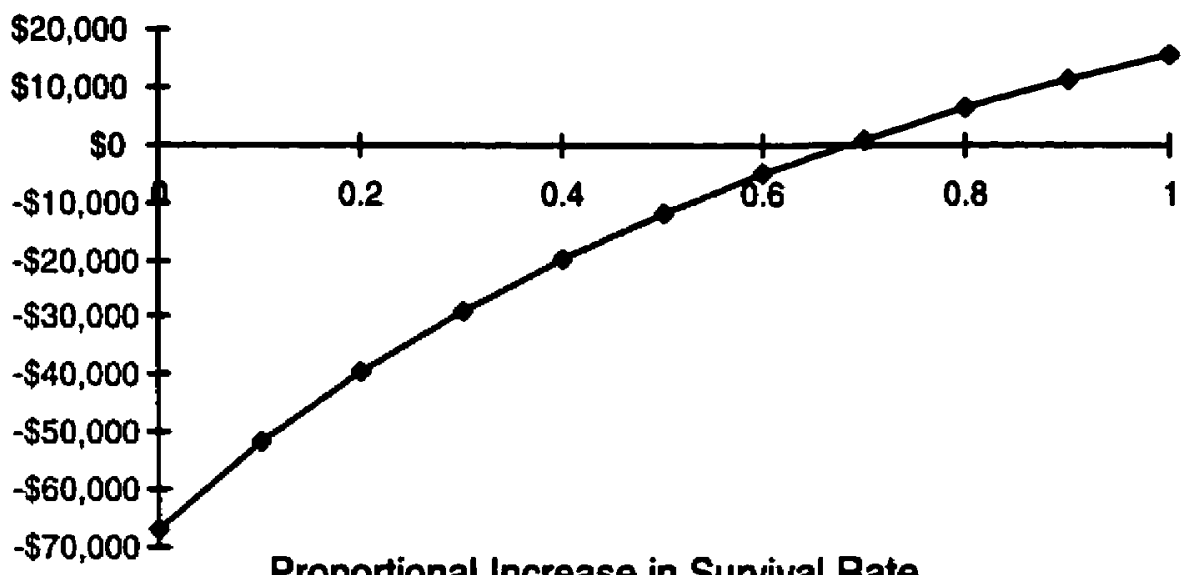

Proportional Increase in Survival Rate 
purely economic perspective, uncertainty about the effects of enhancement on chum salmon is more important in this case study than the uncertainty about the effects of enhancement on coho survival rates. The NPV was also sensitive to the marine survival rate for coho salmon because changes in the marine survival rate affect the entire stock, compared with the effects of a side channel which only affect a proportion of the stock (Figure 13). Finally, the NPV was quite sensitive to the discount rate employed (Figure 14), with discount rates above 3.6 percent producing a net economic loss.

The NPV of a side channel was not consistently positive or negative for the range of parameter values I investigated in these sensitivity analyses. If the sign of the NPV of a side channel was robust to changes in parameter values, then a monitoring program for groundwater-fed side channels would not be wartanted, because we would already be fairly confident that enhancement was either worthwhile or not worthwhile. However, given the uncertainty in many of these parameter values and the sensitivity of the NPV to their values, it was appropriate to consider the benefits of monitoring through a formal decision analysis.

\section{Decision Analysis}

\section{A. Baseline Results}

For the baseline case, which included monitoring programs using "high-precision" sampling methods, the probability of a correct decision increased with the number of systems and number of years monitored (Figure 15). However, as the number of systems monitored increased, the incremental increase in the probability of making a correct decision decreased (i.e. the curves flattened). 
Figure 13. Net present value of a side channel as a function of the coho marine survival rate.

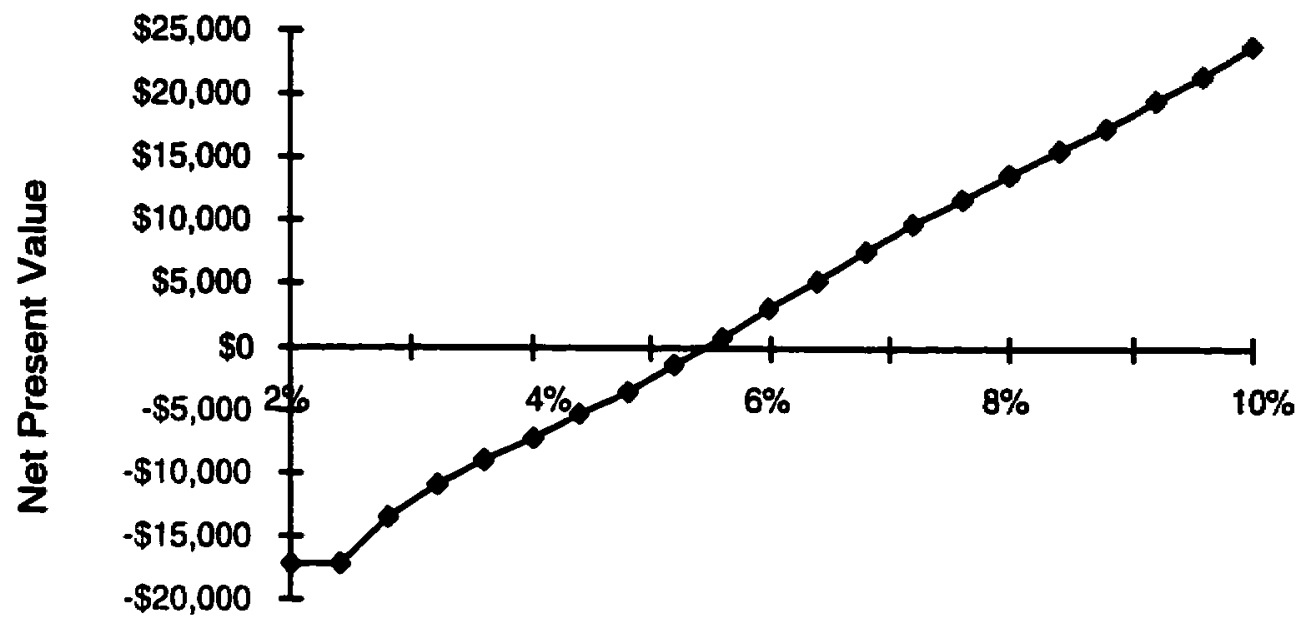

Coho Marine Survival Rate

Figure 14. Net present value of a side channel as a function of the real discount rate.

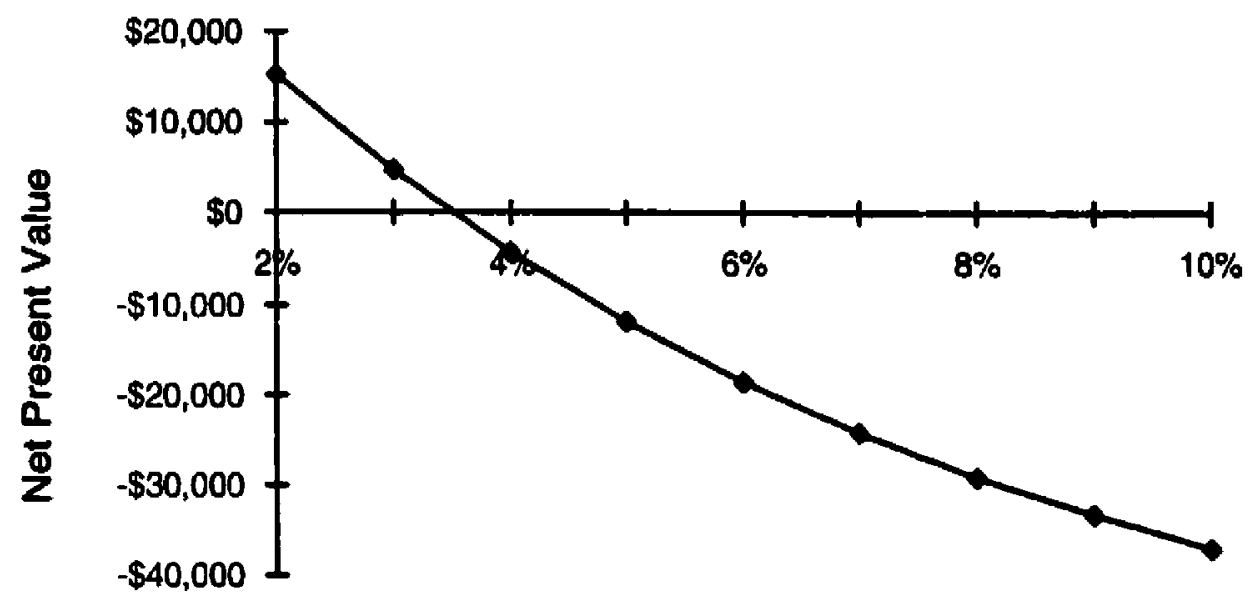

Real Discount Rate 
Figure 15. The probability that monitoring programs of different duration ( $l$ or 2 years) and different numbers of systems ( 1 to 10 systems) lead to a cortect decision regarding whether to apply enhancement more widely with the baseline parameter assumptions.

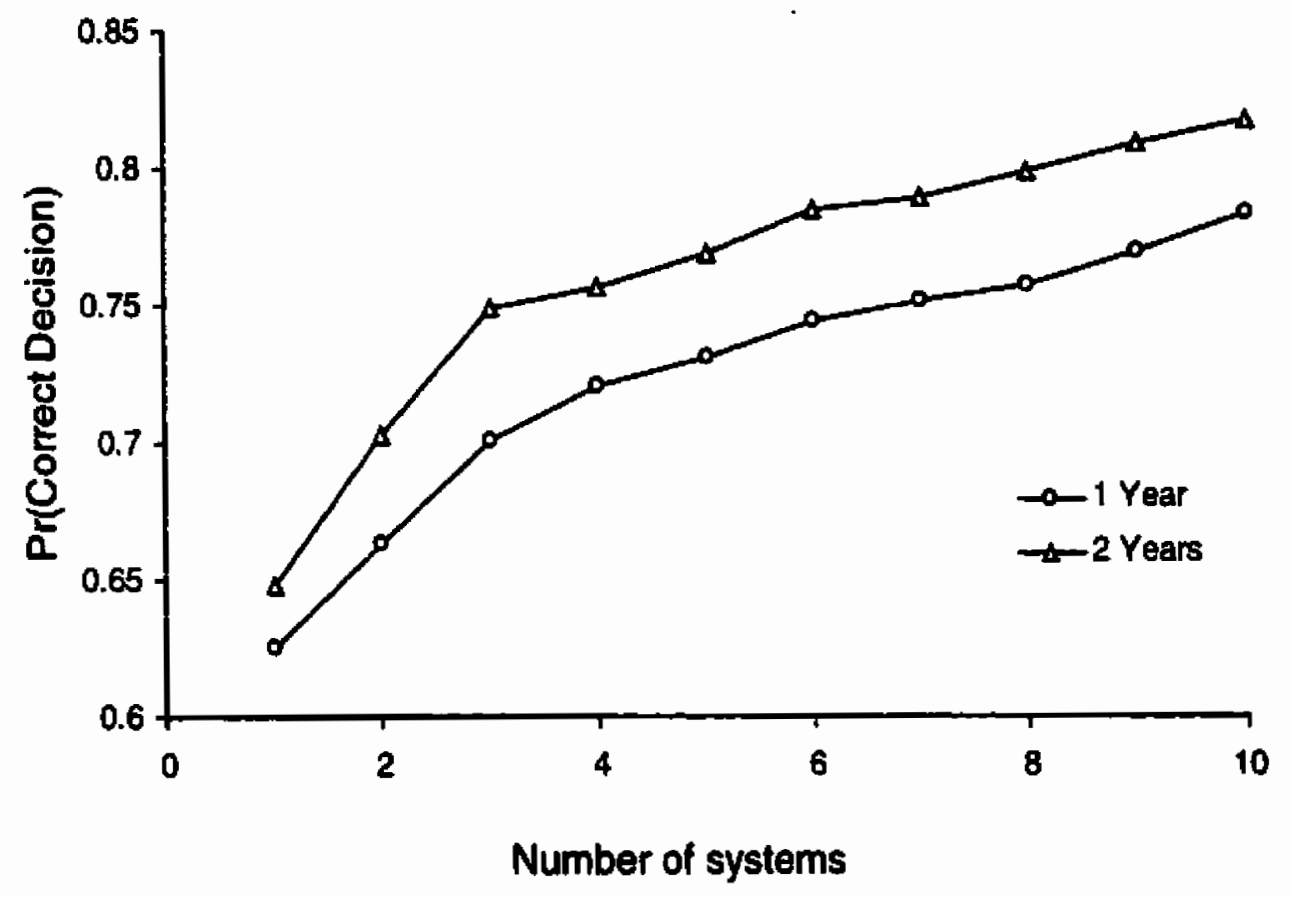


The expected economic benefit of monitoring is the difference between net economic returns from enhancement (incremental fish production minus enhancement costs) for the option with monitoring and the net economic returns for the "enhancement without monitoring" management option. With the high-precision monitoring program and other baseline assumptions, this value is positive for the range of monitoring options investigated (Figure 16). The reason for this result is that for the "enhancement without monitoring" option, enhancement is always undertaken, even when it is not worthwhile to do so. When there is a large probability that enhancement is not worthwhile on purely economic grounds, monitoring prior to making a decision on whether to apply enhancement can provide large economic gains by helping to avoid investing in projects that do not generate sufficient benefits to cover the costs. However, as the number of systems monitored increased, the incremental benefits of each additional system observed decline (Figure 16), because past some point the additional observations did not lead to large improvements in the probability of making a correct decision (Figure 15).

The expected net economic benefit (ENB) of monitoring, which is simply the expected economic benefit of monitoring less the present value of monitoring costs, first increased with the number of systems monitored and then decreased after reaching a peak (Figure 17). This occurred because the expected economic benefit of monitoring exhibited decreasing increments for each additional system monitored, while monitoring costs increased linearly with the number of systems monitored.

The best monitoring options were all options in which the duration of the monitoring program was only one year. There are two reasons for this result. First, I assumed that there are no year-specific effects shared across-systems. However, I 
Figure 16. The expected economic benefit of decisions regarding whether to apply enhancement further for various monitoring programs with baseline parameter assumptions.

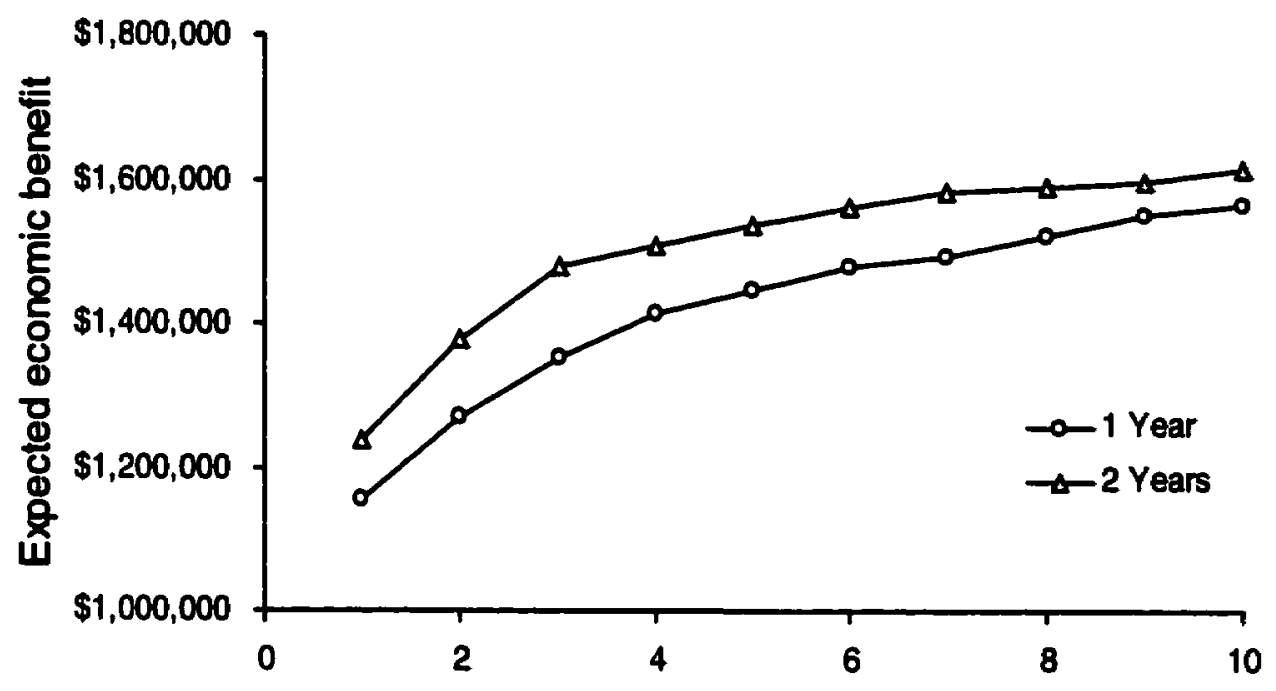

Number of systems

Figure 17. The expected net economic benefit of various monitoring programs with baseline parameter assumptions.

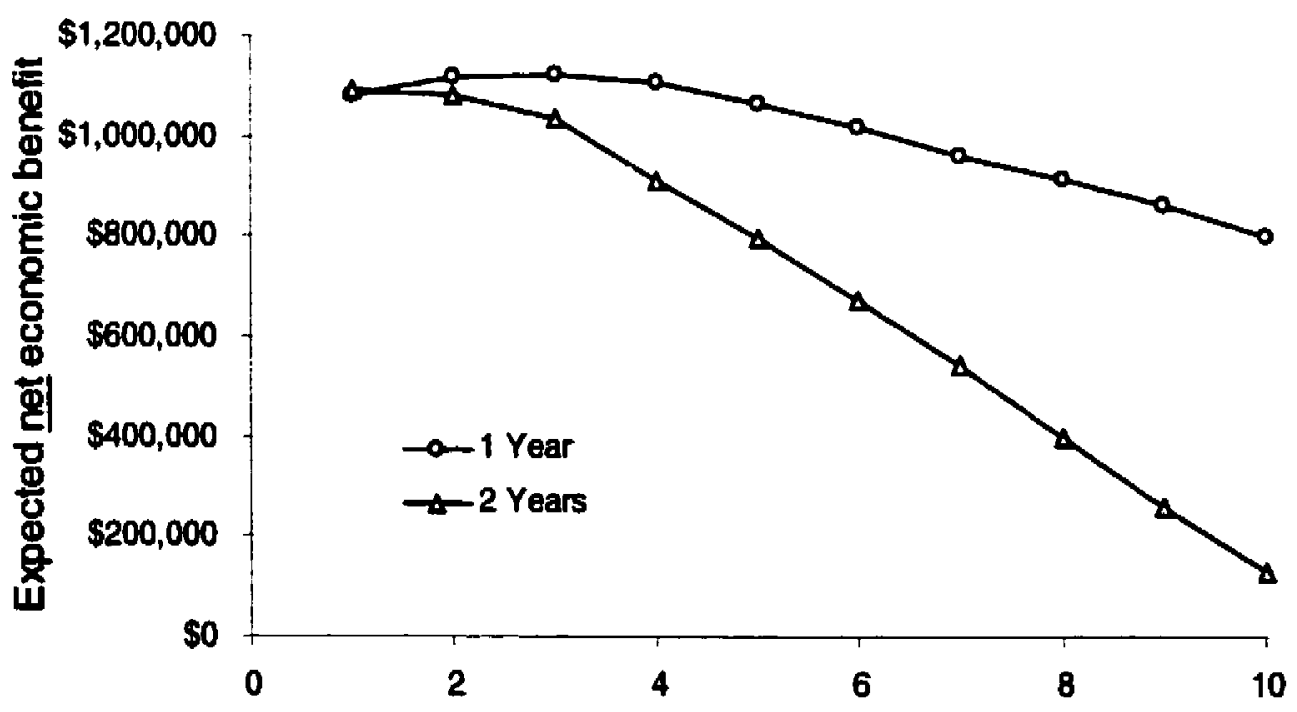

Number of systems 
assumed that there are system-specific effects that are consistent across years. Hence, the observed incremental increases in survival rates (i.e. effect sizes) of fish using side channels may be consistently higher for some systems than for others, due to inherent differences in the quality of wild habitat and/or side channel habitat in these systems but observed effect sizes in all systems are not consistently higher in some years than in others. Therefore, holding all else equal, adding an additional enhanced system to monitor will be more useful for reducing the chances of confounding effects occurring (such as the inherent properties of a given system) than increasing the number of years over which side channels are monitored. A second advantage of a short monitoring program with few years in duration compared to a longer program with fewer systems relates to the discounting of costs and benefits. With a longer monitoring program, the benefits of enhancement (if enhancement is applied) are deferred, thus reducing their present value.

The best monitoring option was to monitor 3 systems for 1 year (Figure 17). Under my baseline assumptions, the probability that the NPV of a side channel is greater than zero is only 0.336 . Hence, the probability that "enhancement without monitoring" is the correct management action is 0.336 , while the probability that the "economically optimal" monitoring program leads to a correct decision is 0.701 . As a result, monitoring prior to proceeding with enhancement yielded an expected net economic benefit of approximately \$1.1 million (Table 13).

While the different monitoring options yielded a positive expected net economic benefit over the "enhancement without monitoring" option, the latter option performed better than either the "economically optimal" or "perfect information" scenario in terms of stock rebuilding goals for coho. This is partially due to the assumption in the decision 
Table 13. Summary of performance measures for decision analysis with baseline parameter values.

Performance Measure

\section{Management Option}

\begin{tabular}{ccc}
$\begin{array}{c}\text { "Economically } \\
\text { optimal" } \\
\text { monitoring } \\
\text { program }\end{array}$ & $\begin{array}{c}\text { "Enhancement } \\
\text { without } \\
\text { monitoring" }\end{array}$ & $\begin{array}{c}\text { Perfect } \\
\text { Information }\end{array}$ \\
\hline
\end{tabular}

Probability of a correct decision

0.701

0.3825

0.336

I

Probability that enhancement is Undertaken

Expected net economic benefit

$\$ 1,123,936$ compared to "enhancement without monitoring"

$\operatorname{Pr}(\text { Recruits } \geq \text { target })^{2}$ by year 7

0.341

0.383

$\operatorname{Pr}$ (Recruits $\geq$ target) by year 21

1

Expected recruits in year 7

117.6

231.1

315.9

$\begin{array}{cc}0.892 & 0.300 \\ 1 & 0.336 \\ 1 & 1 \\ 168.5 & 113.9 \\ 370.8 & 220.6 \\ 390.0 & 310.3\end{array}$

Expected recruits in year 21

T"Economically optimal" monitoring program is 3 systems for $l$ year

${ }^{2}$ Denotes probability that recruits will exceed target by a given year 
model that managers only apply enhancement if the estimated NPV of side channels is greater than or equal to zero. However, at the conclusion of the experiment, managers could still apply enhancement even if the estimated NPV was less than zero based on some biological performance criteria. This would reduce the differences in economic and stock-rebuilding performance measures among the different management options.

The expected number of coho recruits and probability that the number of recruits exceeded recruitment targets in years 7,13 , and 22 (25 percent, 50 percent, and 100 percent maximum wild recruitment in these years) in the post-experimental period were greater when enhancement was undertaken immediately without monitoring (Table 13). In the "enhancement without monitoring" option, enhancement was always applied more widely, whereas enhancement was only applied more widely when the estimated net present value of a side channel exceeded zero with the "economically optimal" monitoring program, and when the true net present value of a side channel exceeded zero with the "perfect information" scenario. In the latter two cases, enhancement was not applied more widely in more than 60 percent $(1,200)$ of the Monte Carlo trials (Figures $18 \mathrm{~A}$ and $18 \mathrm{~B})$. This result combined with baseline assumptions regarding the mean proportional increases in coho egg-to-fry and coho fry-to-smolt survival rates, led to a large number of outcomes in which coho recruitment in any given year was lower (Figures 18A and 18B) than it would have been had enhancement taken place (Figure 18C). It should be noted that the probability that enhancement was undertaken was similar in the "economically optimal" monitoring option and "perfect information" scenario, which led to similar frequency distributions and expected numbers of recruits in any given year (Figure 18A and 18B). 
Figure 18. Frequency distributions for the number of coho recruits in year 7 of the postexperimental period with: (A) the "economically optimal" monitoring program, (B) "perfect information", and (C) "enhancement without monitoring".

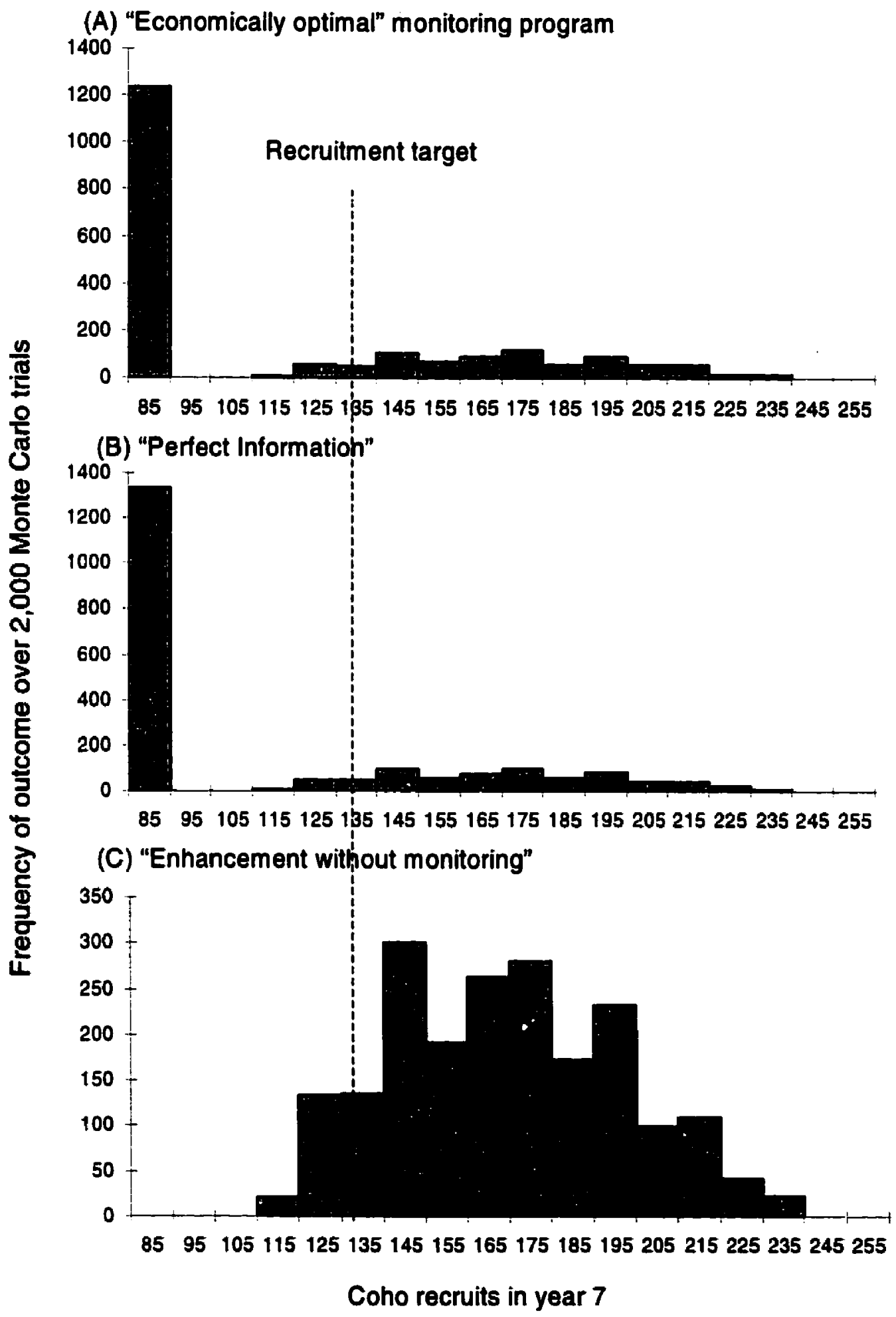


While enhancement did lead to more coho recruits by years 7,13 , and 22 , these additional recruits did not provide a sufficient economic benefit to justify building additional side channels when purely economic criteria are used to judge the efficacy of side channels. The reason for this is that the net present value of each side channel was much more sensitive to the effects of enhancement on chum salmon (Figure 12), due to the low initial coho spawner abundance and low coho marine survival rate. Hence, even when enhancement may have a large effect on coho, unless the effect is also large for chum, additional enhancement is not worthwhile from a purely economic perspective.

\section{B. Sensitivity Analysis}

The following resu!ts from the sensitivity analyses (using the parameter values summarized in Table 12) indicate the sensitivity of the conclusions drawn from the baseline case. In all but one of the scenarios simulated, the expected net economic benefit (ENB) of monitoring exceeded zero (Figure 19). These results indicate that where there is only a choice between these two policy options (i.e. either applying enhancement more widely immediately or first monitoring and then deciding whether to apply enhancement more widely), monitoring can greatly improve the economic performance of management. Below, I summarize results for each of the sensitivity analyses in more detail.

\section{Measurement error of sampling methods}

To investigate the importance of sampling methods for estimating juvenile abundance and, hence, mean effect sizes, I performed the decision analysis for monitoring 
Figure 19. The expected net economic benefit of "economically optimal" monitoring programs for all the scenarios investigated in the decision analysis.

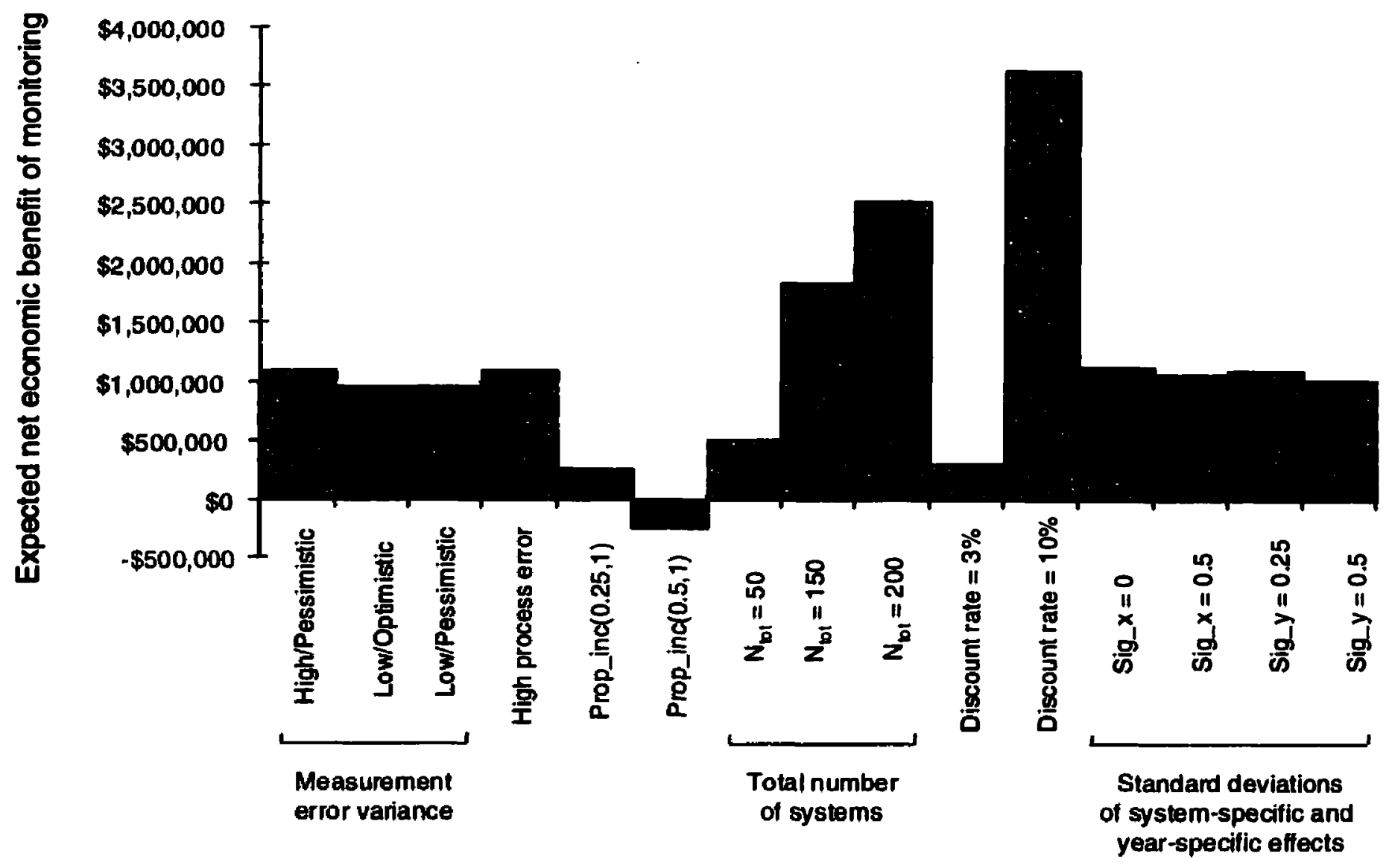


programs using both high-precision and low-precision sampling methods. Due to the uncertainty regarding the precision of these different sampling methods, I derived "optimistic" and "pessimistic" estimates of measurement error variance for abundance estimates for each of the two sets of sampling methods. Hence, a total of four different measurement error scenarios were investigated. The high-precision "optimistic" estimates were the estimates I used for the baseline simulations.

In all cases, the expected net economic benefit of monitoring was positive, thus indicating that monitoring with even very imprecise sampling methods was preferable to the "enhancement without monitoring" option (Figure 20). There was little difference in choice of the "economically optimal" monitoring program for the four measurement error scenarios. In the high-precision, optimistic case, the "economically optimal" monitoring design was to monitor 3 systems for 1 year, while the "economically optimal" monitoring design was to monitor only 2 systems for $I$ year in the other measurement error scenarios.

In all possible comparisons among monitoring designs, the expected net economic benefit of monitoring with the "economically optimal" monitoring program was higher using more precise sampling methods. While low-precision sampling methods were less costly, they introduced enough additional variability into the data that the reduced probability of making a correct decision in the future more than offset any cost savings with those sampling methods. To compare the relative value of different sampling methods, I varied low-precision sampling costs to see how low they would have to be for the expected net economic benefits of monitoring to be equal in the scenarios for highprecision/optimistic and low-precision/optimistic measurement error. I found that the sampling costs per system per year for the low-precision methods would have to be 
Figure 20. Expected net economic benefit of monitoring programs with the "highprecision" and "low precision" sampling methods with optimistic and pessimistic assumptions for measurement error variance.

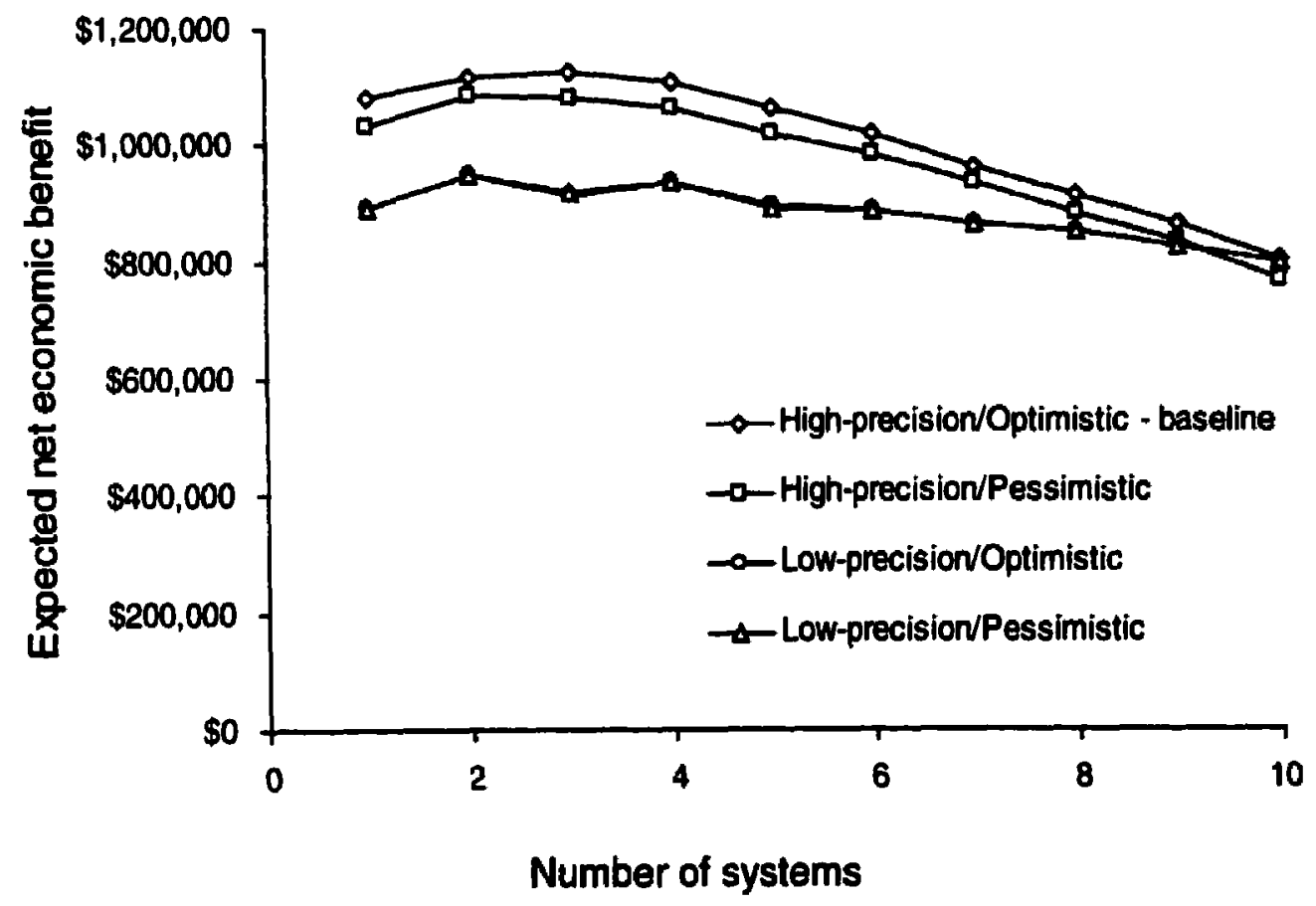


$\$ 20,670$ (compared to the baseline cost estimate for the low-precision sampling program of $\$ 55,000$ ) in order for these two options to be equal. In this case, monitoring 10 systems for 1 year with low-precision sampling methods would yield the same expected net economic benefit of monitoring 3 systems for 1 year using high-precision sampling methods (at a cost $=\$ 80,000$ per system per year).

\section{Process error}

I derived estimates for the process error in observed effect sizes using data on wild survival rates (Bradford, 1995) under two alternative assumptions. In the baseline case, I subtracted "reasonable" estimates of measurement error variance (see Appendix) from interannual variability in estimated survival rates reported by Bradford (1995). To test the importance of this assumption, I also performed the analysis assuming that all of the variability in the data from Bradford (1995) was due to process error. This latter assumption led to higher process error estimates (see Appendix for details), which led to a small reduction in expected net economic benefits of monitoring. This did not have any clear effect on the optimal experimental design for monitoring, however, because monitoring 2,3 , or 4 systems for 1 year yielded close to the same expected net benefit (Figure 21).

\section{Prior probability distributions}

To test the importance of prior beliefs in determining the optimal monitoring program, I conducted a sensitivity analysis on the prior probability distributions for mean effect size on the chum egg-to-fry survival rate, given that I showed previously that it had 
Figure 21. Expected net economic benefit of monitoring programs with high and low process error variance.

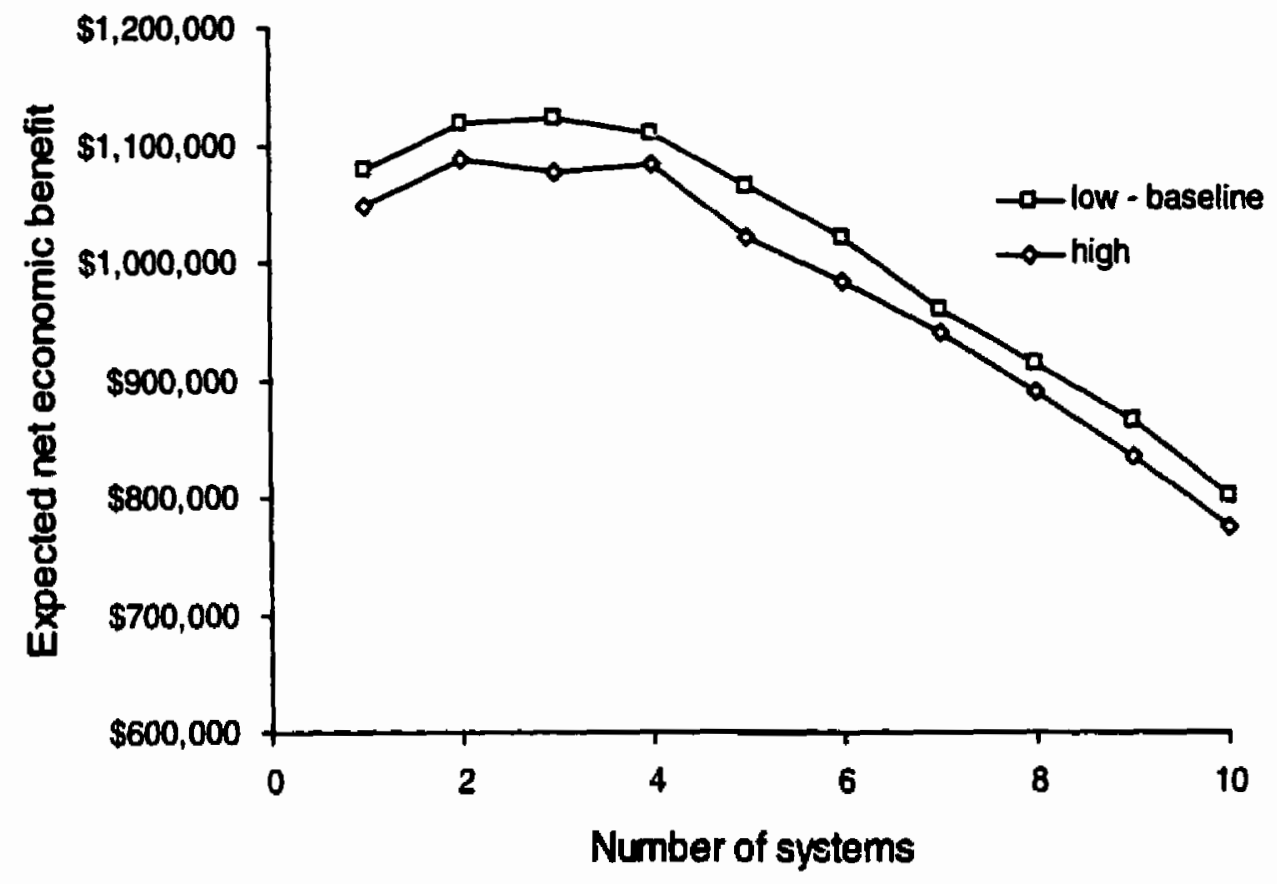


a much larger effect on the economic returns from side channels than did coho survival rates. Specifically, I increased the minimum value for the mean proportional increase in the chum egg-to-fry survival rate from 0 (baseline) to 0.25 and then to 0.5 in a third case, to represent both reduced uncertainty (i.e. smaller possible range) and increased optimism (i.e. higher mean value) in our belief regarding the efficacy of enhancement on chum salmon. These minimum values for the mean proportional increase in the chum survival rate correspond to minimum values for $\mu_{3}$ of $0,0.223$ and 0.405 respectively.

The probability that the net present value of a side channel is greater than or equal to zero, the expected net economic benefit of monitoring, and the expected net economic benefit of having "perfect information", were all very sensitive to the minimum value for $\mu_{3}$ (Table 14). While the increasing optimism regarding the effects of enhancement on chum egg-to-fry survival rates increased the expected net present value (ENPV) of monitoring prior to making a decision whether to apply enhancement more widely, it led to an even larger increase in the ENPV of pursuing the "enhancement without monitoring" option. Hence, the expected net benefits of monitoring fell. In fact, when the minimum mean proportion increase in chum egg-to-fry survival was 50 percent, the expected net economic benefits of monitoring were negative (Figure 22). In this case, proceeding with enhancement right away was the correct decision in a large proportion of Monte Carlo trials, because the probability that the NPV for a side channel exceeded zero was high. Hence, the incremental increase in the probability of making a correct decision with monitoring was smaller than in the other two scenarios.

The biological performance measures were also sensitive to prior beliefs regarding true mean effect sizes (Table 14). As the minimum value of $\mu_{3}$ increased, the probability 
Table 14. Summary of performance measures for decision analysis with different prior probability distributions for the proportional increase in the chum egg-to-fry survival rate $\left(\exp \left(\mu_{3}\right)-1\right)$.

Performance measure

Probability that NPV of side channel $\geq 0$

Expected net economic benefits of "economically optimal" monitoring program

$\operatorname{Pr}($ Recruits $\geq$ target $)$ by year 7

$\operatorname{Pr}($ Recruits $\geq$ target $)$ by year 13

$\operatorname{Pr}(\operatorname{Recruits\geq target)}$ by year $2 \mathrm{l}$

Expected recruits in year 7

Expected recruits in year 13

Expected recruits in year 21
Possible range for the mean proportional increase in chum egg-to-fry survival rates

$$
\left(\exp \left(\mu_{3}\right)-1\right)
$$

Baseline:

\begin{tabular}{lll}
0 to 1 & 0.25 to I & 0.5 to 1 \\
\hline
\end{tabular}

0.336

0.453

0.687

$\$ 1,123,936$

$\$ 255,084$

$-\$ 259,258$

0.341
0.383
1

0.428

0.4735

0.480

0.529

1

1

$\begin{array}{lll}117.6 & 125.6 & 129.7 \\ 231.1 & 253.1 & 264.3 \\ 315.9 & 327.6 & 333.5\end{array}$


Figure 22. Expected net economic benefit of monitoring programs for various assumptions regarding the prior probability distribution for the true mean proportional increase in the chum egg-to-fry survival rate $\left(\exp \left(\mu_{3}\right)-1\right)$.

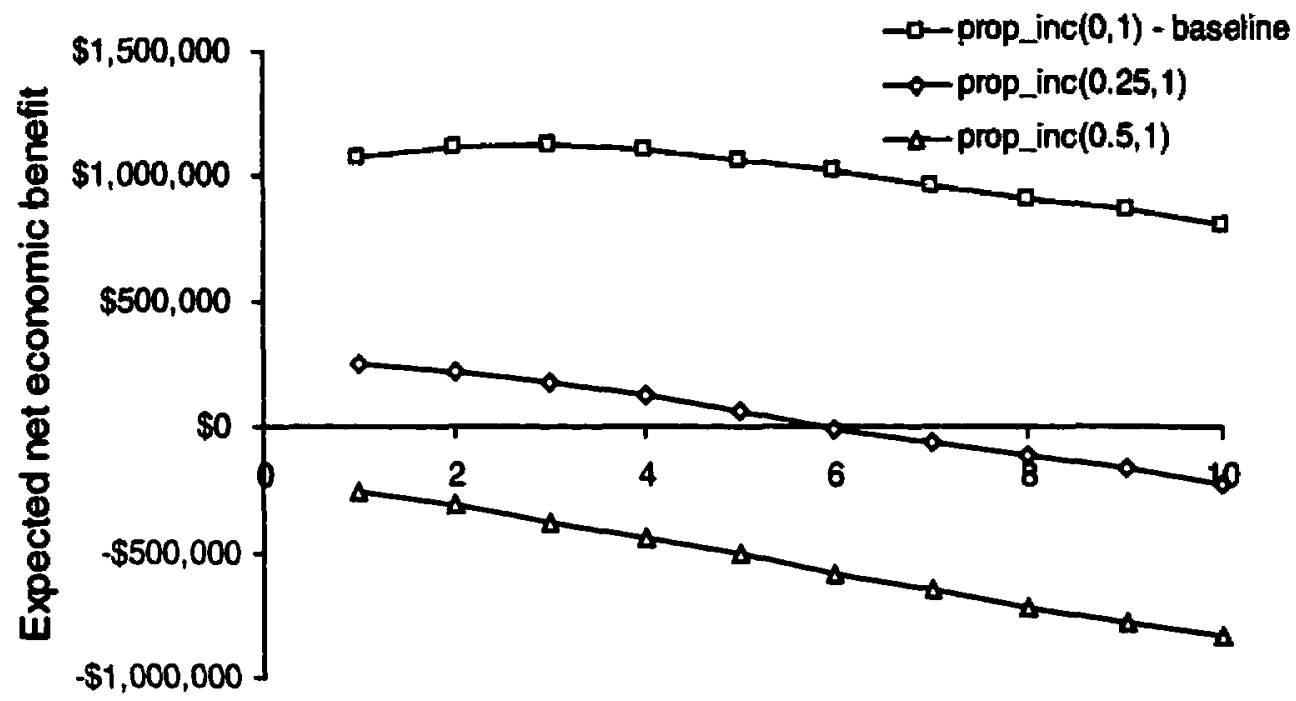

Number of systems

Figure 23. Expected net economic benefit of monitoring programs for various assumptions regarding the total number of non-experimental systems to which the results of the monitoring program can be applied.

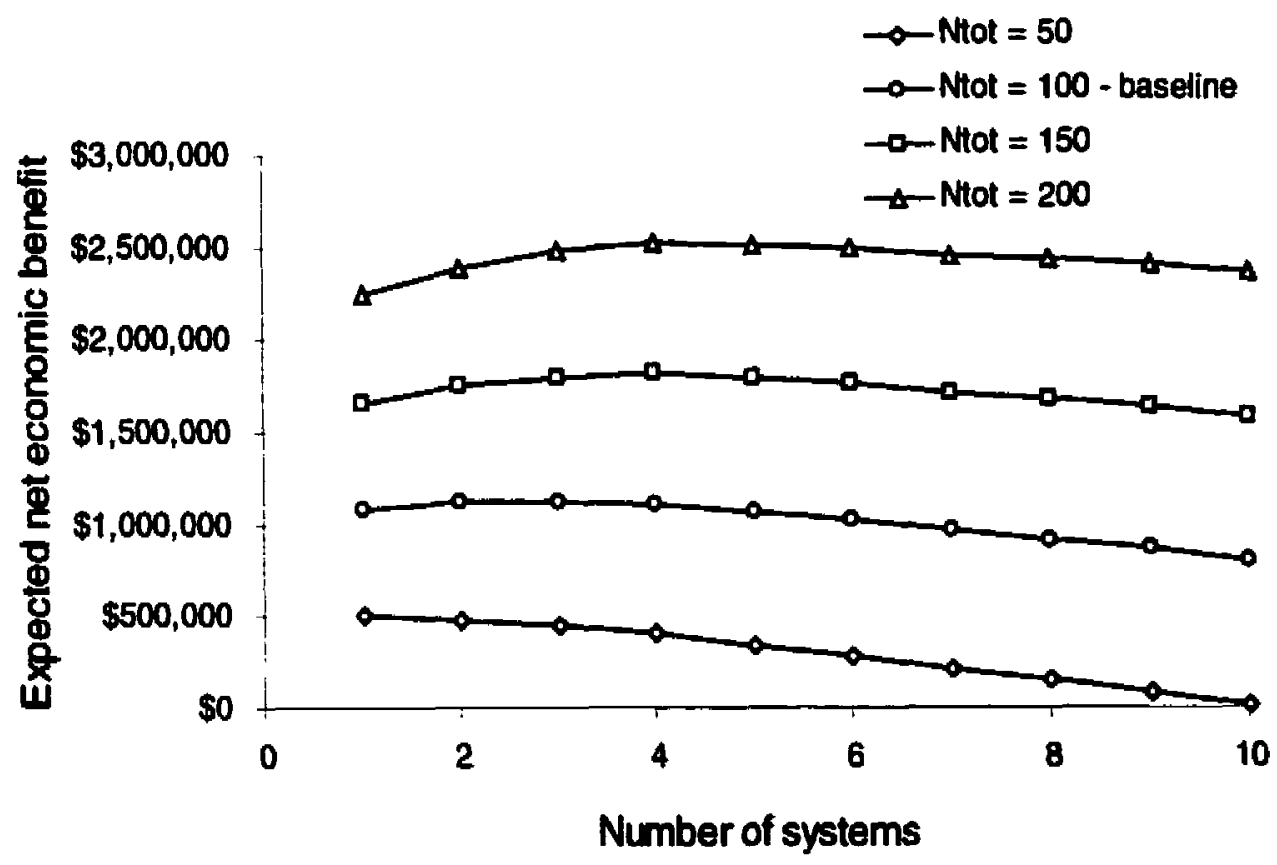


that enhancement is undertaken at the conclusion of the experiment increased and hence, the number of adult coho recruits in each year approached the values that would have been obtained had enhancement proceeded without monitoring.

\section{Number of systems to which enhancement is applied}

The number of systems to which the monitoring results can be applied $\left(N_{t o t}\right)$ is another important factor in determining the value of information from monitoring and hence, the optimal experimental design for monitoring. As the number of systems to which enhancement can be applied increased, so did the expected net benefit of monitoring (Figure 23). The optimal number of systems monitored increased from I $\left(N_{\text {lot }}\right.$ $=50)$, to $3\left(N_{t o t}=100\right)$ to $4\left(N_{t o t}=150\right.$ or 200$)$. In all cases, monitoring prior to proceeding with enhancement yielded positive expected net economic benefits.

\section{Real discount rate}

Another important consideration is the real discount rate, which reflects the tradeoff between present and future benefits. The choice of discount rate is especially important when evaluating habitat enhancement measures, such as groundwater-fed side channels, in which the majority of costs are incurred up front, while the benefits are spread over a long period in the future. In such cases, increasing the discount rate increases the relative weighting of costs and benefits closer to the present, thus making enhancement less attractive.

Not surprisingly, the discount rate had a very large impact on expected net economic benefits of monitoring, which increased dramatically when I increased the 
Figure 24. Expected net economic benefit of monitoring programs with different real discount rates.

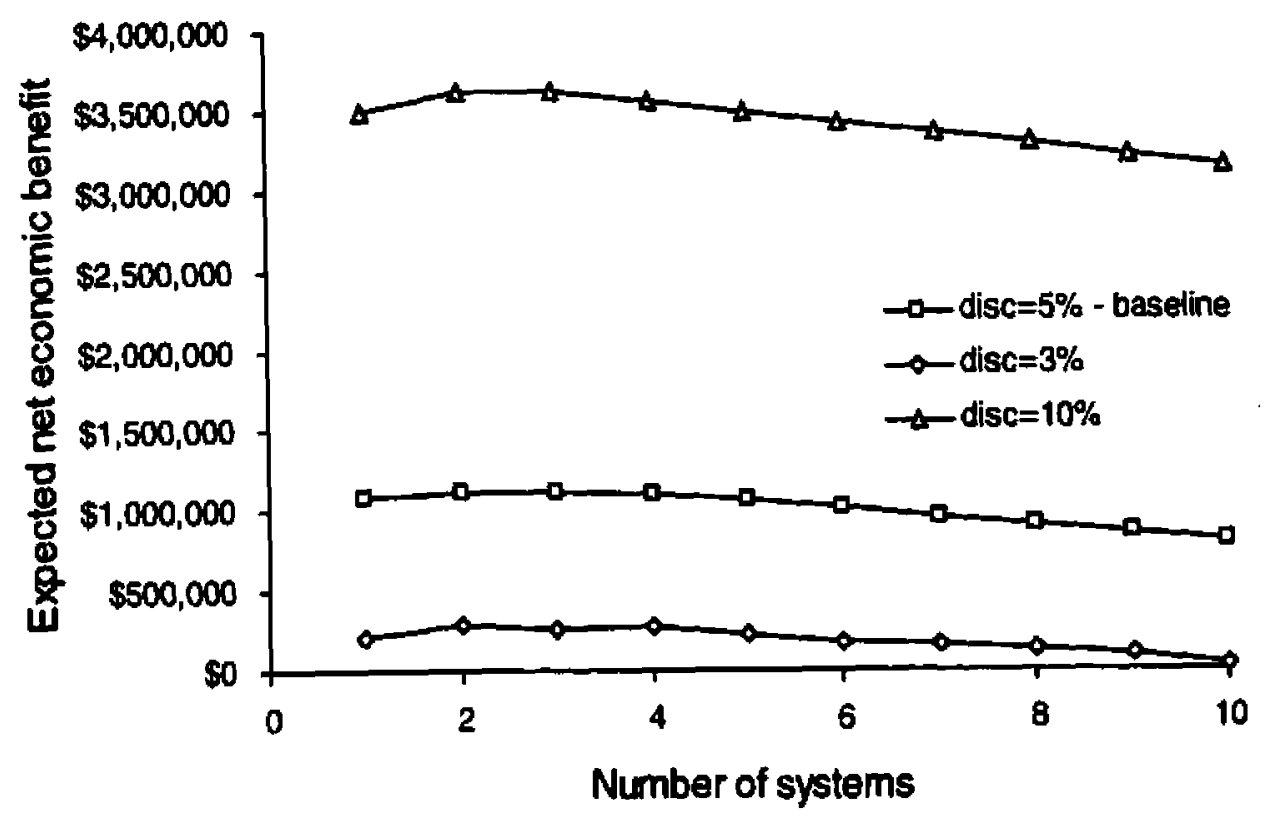


discount rate to 10 percent from 5 percent. Conversely, the benefits of monitoring fell when I lowered the discount rate to 3 percent (Figure 24). However, in the latter case, the expected net economic benefits of monitoring were still positive.

The reason for this result is that for lower discount rates, the net present value of a side channel is more likely to be positive. Hence, the probability that "enhancement without monitoring" is a correct decision increases (Table 15). Conversely, with a discount rate of 10 percent, the net present value of a side channel is never positive for the range of effect sizes I investigated. Hence, any case in which enhancement is not undertaken is an economic gain relative to the "enhancement without monitoring" option.

However, the optimal design for monitoring was not very sensitive to changes in the discount rate. The "economically optimal" monitoring programs were to monitor between 2 and 4 systems for 1 year.

\section{Variance of system-specific effects}

Increasing the variance of system-specific effects (i.e. differences among systems) as summarized in Table 9 reduced the expected net economic benefit of monitoring (Figure 25). The reason for this result is that the increased variation in data due to greater variation in system-specific effects reduced the probability that a given monitoring option could correctly identify whether enhancement was worthwhile. However, in all cases, the expected net benefit of monitoring was positive.

Surprisingly, the optimal experimental design did not change when I changed the variance of system-specific effects. In all three cases, the optimal experimental design was to monitor 3 systems for 1 year. This lack of predictable patterns is caused by two 
Table 15. Summary of performance measures for decision analysis with different real discount rates.

Performance measure

\begin{tabular}{|c|c|c|c|}
\hline & $\begin{array}{l}\text { Baseline: } \\
5 \text { percent }\end{array}$ & $\begin{array}{l}\text { Low: } \\
3 \text { percent }\end{array}$ & $\begin{array}{l}\text { High: } \\
10 \text { percent }\end{array}$ \\
\hline $\begin{array}{l}\text { Probability that NPV of side } \\
\text { channel } \geq 0\end{array}$ & 0.336 & 0.5715 & 0 \\
\hline $\begin{array}{l}\text { Expected net economic } \\
\text { benefits of "economically } \\
\text { optimal" monitoring } \\
\text { program }\end{array}$ & $\$ 1,123,936$ & $\$ 283,667$ & $\$ 3,629,684$ \\
\hline $\operatorname{Pr}($ Recruits 2 target $)$ by year 7 & 0.341 & 0.472 & 0 \\
\hline $\operatorname{Pr}($ Recruits $\geq \operatorname{target})$ by year 13 & 0.383 & 0.524 & 0 \\
\hline $\operatorname{Pr}($ Recruits $\geq \operatorname{target})$ by year 21 & 1 & 1 & 1 \\
\hline Expected recruits in year 7 & 117.6 & 129.5 & 88.7 \\
\hline Expected recruits in year 13 & 231.1 & 263.6 & 153.2 \\
\hline Expected recruits in year 21 & 315.9 & 332.8 & 274.8 \\
\hline
\end{tabular}

Real discount rate 
Figure 25. Expected net economic benefit of monitoring programs for various assumptions regarding the standard deviation of system-specific effects.

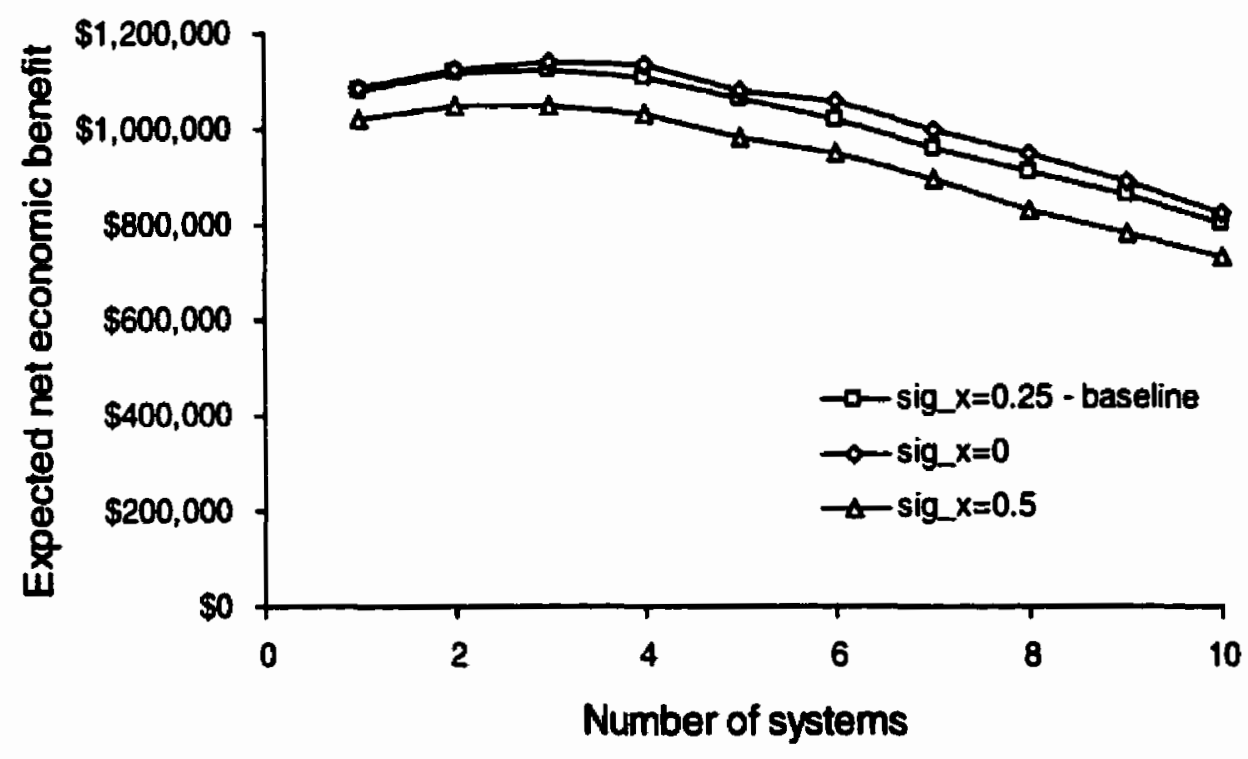


factors: (1) the relatively large estimates of monitoring costs and (2) the large estimates of process error. When using estimates of measurement error consistent with the assumptions about measurement error variance for this study, the process error variances came out to be very large relative to the other sources of variation.

\section{Variance of year-specific effects}

Increasing the variation in year-specific effects (i.e. year-to-year variation shared across systems) reduced the expected net economic benefits of all monitoring programs (Figure 26). However, despite this negative effect, in all cases, the expected net economic benefit of the "economically optimal" monitoring program was still positive (Figure 19).

Changes in the magnitude of year-specific variation also affected the optimal monitoring design. In the baseline case (Figure 26A), in which it was assumed that there were no year-specific effects, the optimal monitoring program was to monitor 3 systems for only 1 year. However, when the standard deviation of year-specific effects was increased from 0 to 0.25 , monitoring options with 2 systems and 1 year and 1 system and 2 years yielded similar expected net benefits (Figure 26B), and when I increased it further to 0.5 , the optimal monitoring program was to monitor 1 system for 4 years (Figure 26C).

The increase in the number of years monitored in the "economically optimal" monitoring program with larger year-specific effects is the result of larger differences in observed responses to enhancement from year-to-year. For example, in one year, survival rates of wild fish may be poor in all systems due to adverse flow conditions caused by 
Figure 26. Expected net economic benefit of monitoring programs of different durations (I to 4 years) with: (A) no year-specific effects, (B) moderate variability in year-specific effects, and (C) high variability in year-specific effects.

(A) No variation in year-specific effects .- baseline

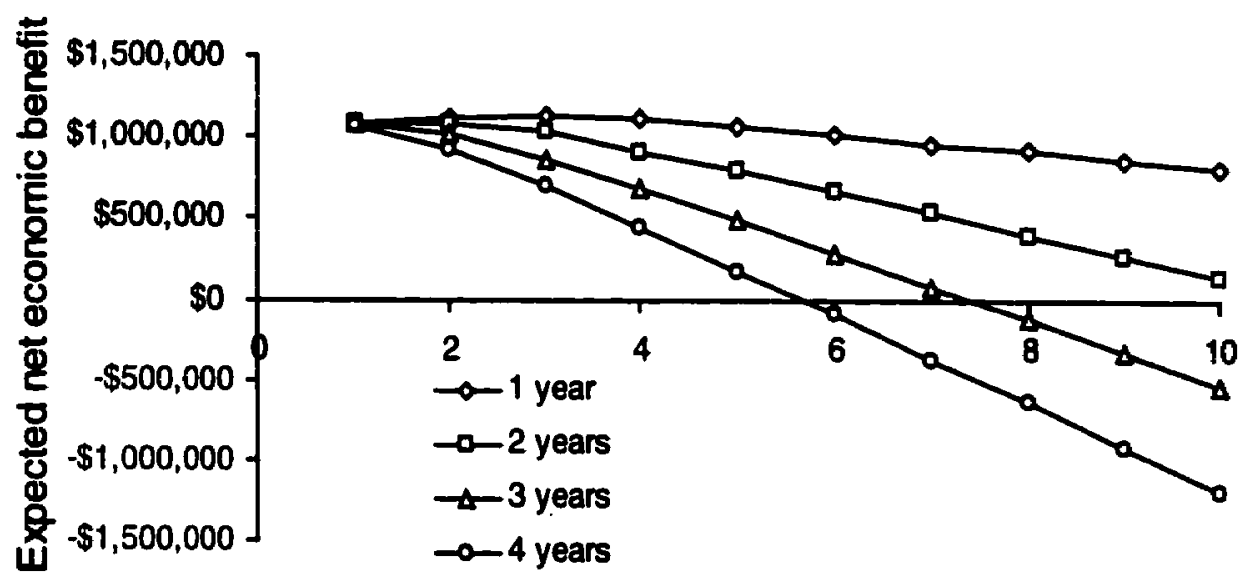

(B) Moderate variation in year-specific effects

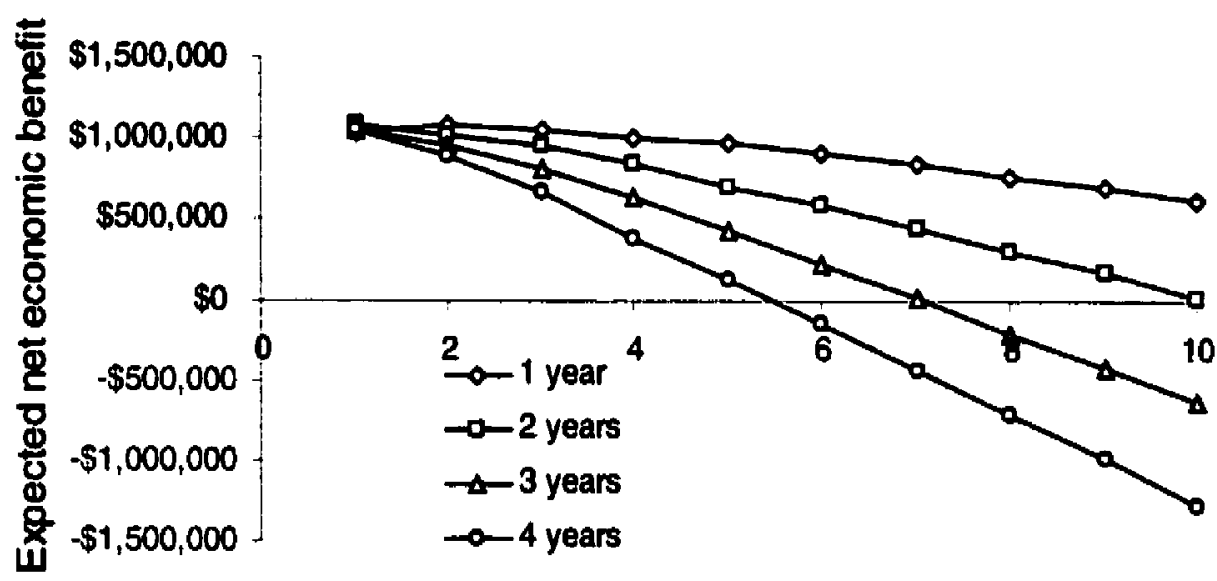

(C) High variation in year-specific effects

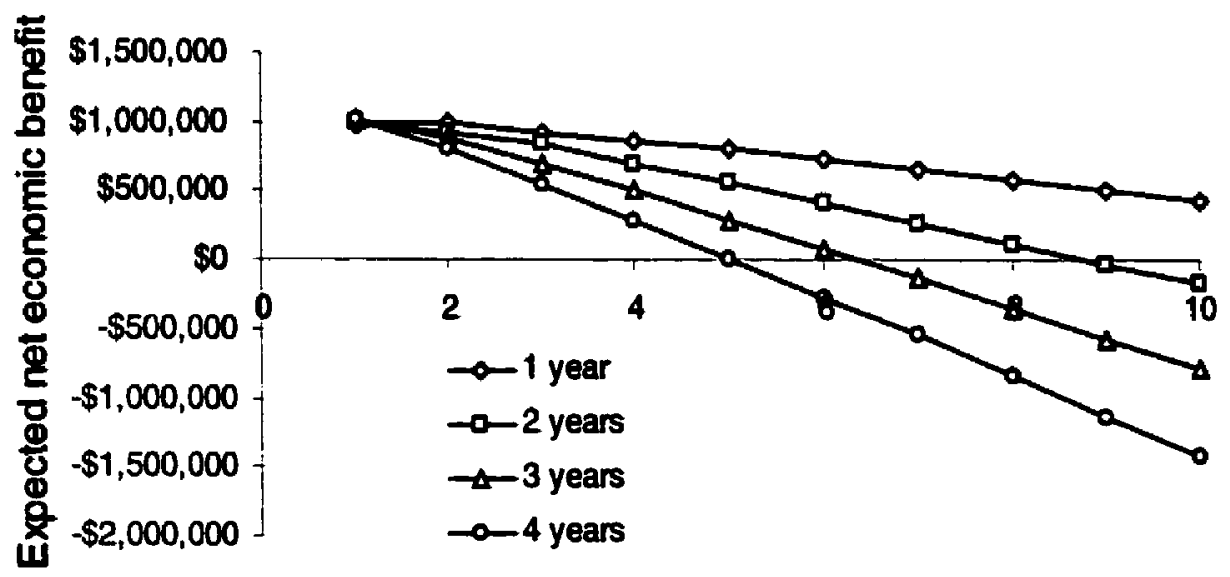

Number of systems 
large-scale climatic processes, while survival rates may be relatively unaffected in side channels because these may act as a buffer against such environmental fluctuations. In this case, the observed effect sizes (incremental increases in survival rates) may be consistently high across all systems, while observed effect sizes might be lower in years without such adverse conditions. When large potential differences exist in observed effect sizes from year-to-year, a few years in which wild survival rates are poor, or in which wild survival rates are high, could be sampled by chance. Increasing the number of years monitored reduces the chance that such large year-specific effects could lead to large errors in estimates of mean effect sizes. Hence, the relative value of monitoring additional years versus additional systems increases as the variance of year-specific effects increases.

\section{GENERAL DISCUSSION}

The results of this analysis are similar to the findings of Walters and Green (1997), which indicate that short monitoring programs are optimal. Such programs are likely to have low statistical power, but they perform better than longer monitoring programs for two reasons. First, in most simulations performed, I assumed that there are no year-specific effects of enhancement that are shared by all monitored systems (i.e. $\left.\sigma_{Y, 1}=\sigma_{Y, 2}=\sigma_{Y, 3}=0\right)$. Furthermore, $[$ assumed that there are effects unique to each system that occur in all years (i.e. system \#1, for instance, may show a much larger response to enhancement than system \#2, and this difference between system-specific responses recurs every year). Hence, holding all other factors equal, adding an additional system to the monitoring program provides more information than adding another year, 
because it helps to prevent unique system-specific responses from confounding the monitoring results. Second, I applied a positive discount rate. Hence, by deferring the decision of whether to enhance further until a later date, the net value of benefits from enhancement is reduced.

Keeley and Walters (1994) found that monitoring programs with 8 to 16 pairs of watersheds and 4 to 8 years in duration were optimal for monitoring watershed restoration projects. Their results suggest a much larger monitoring program than those suggested by this analysis. There are a number of assumptions that led to this result.

First, monitoring costs in their model are much smaller relative to the assumed benefits from each project than is the case in my analysis. In the case of side channels for coho and chum salmon, the costs of monitoring one system for one year in my analysis $(\$ 80,000)$ are slightly greater than the costs of constructing a $2,000 \mathrm{~m}^{2}$ side channel $\left(2,000 \mathrm{~m}^{2} \times \$ 35.98 / \mathrm{m}^{2}=\$ 71,960\right)$, whereas Keeley and Walters (1994) assumed monitoring costs were only 6 percent of capital costs! Second, they assume a dichotomous state of nature in which the true response of each watershed is either very high (with a large positive NPV) or very low (with a large negative NPV). In contrast, I assume a continuous range of possible responses of salmon to enhancement, hence, the true NPV can be very close to zero. Thus, in Walters' and Keeley's case, the costs of making an incorrect decision are always very large, while with my assumptions, costs of incorrect decisions may be large or small. Keeley and Walters'(1994) approach tends to increase the value of information relative to the costs of obtaining it, hence suggesting a larger monitoring program. Finally, they assume that there are year-specific effects that are shared across watersheds (i.e. $\sigma_{Y}>0$ ), which increases the need for monitoring 
additional years to prevent responses unique to each year (and shared across systems) from confounding monitoring results. However, even when I assumed that such effects existed, "economically optimal" monitoring programs were only 2 to 4 years in duration and the number of systems monitored was reduced, owing to the high costs of monitoring in my case.

For most of the range of parameter values that I investigated through sensitivity analysis, the expected net economic benefits of monitoring were positive, thus indicating that monitoring programs may improve the economic performance of management (Figure 19). However, this result is highly contingent upon the amount of uncertainty and optimism regarding the effects of enhancement. Sensitivity analyses on the prior probability distributions for effect sizes indicated that when there is more optimism regarding the efficacy of enhancement and less uncertainty, a non-experimental approach might be preferable. This point is especially important for small-scale enhancement projects, such as side channels, where the costs of monitoring each project can be quite large compared to the costs and potential direct benefits of enhancement.

An additional benefit that I have not quantified is the reduction in economic risk due to monitoring programs. Evaluating projects based on their expected net benefits implicitly assumes that decision makers are risk-neutral. However, if decision makers are averse to economic risk, this further increases the attractiveness of the different monitoring options relative to the "enhancement without monitoring" case, because they reduce the chances that large and unproductive capital investments would be made.

Sensitivity analyses on the sampling methods used and their associated measurement errors indicate that "high-precision" sampling methods outperform the 
"low-precision" sampling methods (as defined in Tables 7 through 10) in terms of their expected net economic benefits. While the costs of using "low-precision" sampling methods might be lower, the additional variability in the data introduced by the higher measurement errors (and hence the reduced value of information) is more likely to offset any savings in terms of monitoring costs.

Although I have not calculated the statistical power for the optimal experimental designs in this analysis, the small number of systems and short durations of the "economically optimal" designs suggest that these designs will have low power for statistical hypothesis tests. However, the statistical hypothesis testing framework may be inappropriate for making decisions regarding the size of optimal monitoring programs. The main reason is that the hypothesis testing framework does not take into account the costs of obtaining information and the relative long-term benefits and costs of making correct and incorrect inferences. Statistical power analysis only provides information on the probabilities of making correct inferences for a given true effect size.

Due to lack of data, numerous assumptions were required to complete this analysis. While I have tested many of the most important assumptions through sensitivity analysis, there are many more that could have an effect on the decision analysis. Below, I discuss some (but not all) of these assumptions:

1. Independence of $\mu_{1}, \mu_{2}, \mu_{3}$-For each Monte Carlo trial, values for true mean effect sizes were drawn independently from their uniform probability distributions. However, this may not necessarily reflect reality regarding true mean effect sizes. For example, if we believe that the true mean effect on the coho egg-to-fry survival rate is large (i.e. $\mu_{I}$ is large), then we might expect that the true mean effect size on the chum 
egg-to-fry survival rate is also large (i.e. $\mu_{3}$ is large). This would be reflected in the model by making sets of simulated true mean effect sizes positively correlated, which would have the effect of increasing the variance of the true NPV of enhancement. It is not clear, however, what effect this would have on the choice of experimental design.

2. Chum benefits - The benefits for chum salmon were calculated assuming that side channels can produce 200 fry per $\mathrm{m}^{2}$ of side channel. However, if side channels are tailored for coho, the number of chum fry produced per $\mathrm{m}^{2}$ might be even lower. This would probability that enhancement is worthwhile and, hence, increase the expected net economic benefits of monitoring prior to making a decision regarding future enhancement.

3. Univariate Sensitivity Analysis - I performed my sensitivity analyses with respect to one parameter at a time (or one group of parameters with a qualitatively similar effect in the model), but there could be interactions among parameters. However, these are unlikely to change the qualitative direction of effects that each individual parameter has in the model.

4. Universe of inference - The number of systems to which monitoring results are applied $\left(N_{t o t}\right)$ may be treated as an input in the decision analysis. For example, DFO may choose one monitoring program with results applied to all possible sites (large $\left.N_{t o t}\right)$. However, heterogeneity among sites may lead to large location-specific effects that obscure the true effects of enhancement. An alternative management plan might be to sub-divide all sites into smaller more homogenous sub-groups or strata based on their characteristics and to conduct monitoring separately for each group. This would 
reduce the variance of system-specific effects, thus increasing the precision with which mean effect sizes could be estimated for a given monitoring design. However, I have not investigated this option quantitatively.

5. Bias in sampling methods - I have evaluated monitoring programs using different sampling methods based on the relative precision of these sampling methods. However, sampling methods may also differ in terms of the bias that they produce in parameter estimates. In my analysis, I assumed that all sampling methods produce unbiased estimates of true mean effect sizes, because I did not have sufficient information to determine the bias (if any) that exists for different sampling methods.

6. Direct versus indirect economic benefits - I only took into account the direct economic benefits of enhancement resulting from the increased incremental catches from stocks directly affected by enhancement. However, in certain cases, there may be indirect benefits that should be taken into account. For example, the rebuilding of depressed coho stocks in British Columbia might create benefits in fisheries on other species if those fisheries that are currently closed due to conservation concerns for coho are then reopened.

The effect on this analysis of incorporating such indirect economic benefits to other fisheries would depend strongly on the relative magnitude of the economic costs of enhancement and the potential economic benefits from other fisheries. For example, if the economic benefits from other fisheries are very large compared to the costs of enhancement, this would favour the "enhancement without monitoring" strategy. Conversely, if the costs of enhancement are large relative to potential indirect benefits, monitoring before deciding would be favoured. It should be noted, 
however, that this relies on the presumption that enhancement at worst would have no effect on coho rebuilding, as I have assumed in this analysis. While this assumption might be valid for side channels and other forms of habitat enhancement used for coho saimon, it might not be true for other methods of enhancement such as rearing coho juveniles in hatcheries. In that case, where enhancement may have potentially adverse effects on coho rebuilding, the benefits of monitoring first would increase relative to applying enhancement immediately to all stocks without first monitoring to determine its effects.

\section{Summary and Conclusions}

Wise investment in enhancement projects for Pacific salmon requires rigorous evaluation of their performance through monitoring. However, the costs of monitoring the biological effects of enhancement can be quite large. Given the potentially high costs of monitoring, managers are faced with difficult choices regarding the length of monitoring programs, the number of systems that should be monitored, and the types of sampling methods that should be used.

In this study, I developed a decision-analysis framework to aid in the selection of appropriate experimental designs for monitoring enhancement projects for Pacific salmon. This framework explicitly evaluates different monitoring options based on their costs and the benefits derived from the information they provide. Furthermore, it can be used to determine whether monitoring should be undertaken at all prior to deciding whether to use a particular method of enhancement on a large scale.

I applied the decision analysis framework to groundwater-fed side channels utilized 
by coho and chum salmon. Results from this analysis indicate that monitoring prior to making a decision about whether to apply enhancement on a wider scale (i.e. to all stocks in which it is applicable) is generally preferable to proceeding with enhancement without first monitoring to determine whether it is worthwhile. However, due to the large costs of monitoring relative to the potential costs and benefits of each side channel, optimal monitoring programs were small (in both space and time). The implied low statistical power of such monitoring programs suggests that the benefits of monitoring programs with high statistical power may not be sufficient to outweigh their costs. 


\section{References}

Baranski, Chuck. 1989. Coho Smolt Production in Ten Puget Sound Streams. Technical Report No. 99. State of Washington Department of Fisheries.

Bonnell, R.G. 1991. Construction, operation, and evaluation of groundwater-fed channels for chum salmon in British Columbia. American Fisheries Society Symposium 10; $109-124$.

Bradford, M.J. 1995. Comparative review of Pacific salmon survival rates. Canadian Joumal of Fisheries and Aquatic Sciences 52: 1327-1338.

Bradford, M.J. 1998. A Risk Assessment for Thompson River Coho Salmon. PSARC Working Paper S98-18.

Bradford, M.J., 1999. Temporal and Spatial Trends in the Abundance of Coho Salmon Smolts from Western North America. Transactions of the American Fisheries Society 128: 840-846.

Bradford, M.J., R.A. Myers, and J.R. Irvine. 2000. Reference points for coho salmon (Oncorhynchus kisutch) harvest rates and escapement goals based on freshwater production. Canadian Journal of Fisheries and Aquatic Sciences 57: 677-686.

Bradford, M.J., G.C. Taylor, and J.A. Allan. 1997. Empirical review of coho smolt abundance and the prediction of smolt production at the regional level. Transactions of the American Fisheries Society 126: 49-64.

Brown, T.G. and G.F. Hartman, 1988. Contribution of seasonally flooded lands and minor tributaries to the production of coho salmon in Carnation Creek, British Columbia. Transactions of the American Fisheries Society 117: 546-551.

Clemen, R.T. 1996. Making Hard Decisions: An Introduction to Decision Analysis. 2nd edition. Duxbury Press, Wadsworth Publ. Co., Belmont, California.

Coronado, C. and R. Hilborn. 1998. Spatial and temporal patterns affecting survival of coho salmon (Oncorhynchus kisutch) in the Pacific Northwest. Canadian Journal of Fisheries and Aquatic Sciences 55: 2067-2077.

Cousens, N.B.F., G.A. Thomas, C.G. Swann, and M.C. Healey. A review of salmon escapement estimation techniques. Canadian Technical Report of Fisheries and Aquatic Sciences No. 1108.

Cowan, L. 1991. Physical characteristics and intragravel survival of chum salmon in developed and natural groundwater channels in Washington. American Fisheries Society Symposium 10: 109-124. 
Decker, A.S. 1998. Influence of off-channel habitat restoration and other enhancement on the abundance and distribution of salmonids in the Coquitlam River prepared for B.C. Hydro and the Department of Fisheries and Oceans.

Devore, J.L. 1991. Probability and statistics for engineering and the sciences. Duxbury Press, Belmont California.

Gelman, A., J.B. Carlin, H.S. Stern, and D.B. Rubin. 1995. Bayesian data analysis. Chapman and Hall, London.

Gowan, C. and K.D. Fausch. 1996. Long-term demographic responses of trout populations to habitat manipulations in six Colorado streams. Ecological Applications 6(3): 931-946.

Gowan, C., M.K. Young, K.D. Fausch, and S.C. Riley. 1994. Restricted movement in resident stream salmonids: a paradigm lost? Canadian Journal of Fisheries and Aquatic Sciences 51: 2626-2637.

Hankin, D.G. and G.H. Reeves. 1988. Estimating total fish abundance and total habitat area in streams based on visual estimation methods. Canadian Journal of Fisheries and Aquatic Sciences 45: 834-844.

Hartman, G.F., D.B. Tripp and T.G. Brown. 1998. Overwintering habitats and survival of juvenile salmonids in coastal streams of British Columbia in Carnation Creek and the Queen Charlotte Islands fish/forestry workshop: Applying 20 years of coastal research to management solutions, Hogan and Tschaplinski eds. B.C. Ministry of Forests. Land Management Handbook No. 41.

Hayes, D.B., C.P. Ferreri, and W.W. Taylor. 1996. Linking fish habitat to their population dynamics. Canadian Journal of Fisheries and Aquatic Sciences 53(Suppl. 1): 383-390.

Irvine, J.R., R.C. Bocking, K.K. English, and M. Labelle. 1992. Estimating coho salmon spawning escapements by conducting visual surveys in areas selected using stratified random and stratified index sampling designs. Canadian Journal of Fisheries and Aquatic Sciences 49: 1972-1981.

Irvine, J.R., K. Wilson, B. Rosenberger, and R. Cook. 1999. Stock Assessment of Thompson/Upper Fraser River Coho Salmon. Canadian stock assessment secretariat. Research document 99/28. Fisheries and Oceans Canada.

Jones, M.L., R.G. Randall, D. Hayes, W. Dunlop, J. Imhof, G. Lacroix, and N.J.R. Ward. 1996. Assessing the ecological effects of habitat change: moving beyond productive capacity. Canadian Journal of Fisheries and Aquatic Sciences 
53(Suppl. 1): 446-457.

Koning, C.W. and E.R. Keeley 1997. Salmonid biostandards for estimating production benefits of fish habitat rehabilitation techniques. In P.A. Slaney and D. Zaldokas (eds.) Fish Habitat Rehabilitation Procedures, Watershed Restoration Technical Circular No. 9. British Columbia Ministry of Environment, Lands and Parks and Ministry of Forests.

McAllister, M.K. and R.M. Peterman. 1992. Decision Analysis of a Large-Scale Fishing Experiment Designs to Test for a Genetic Effect of Size-Selective Fishing on British Columbia Pink Salmon (Oncorhynchus gorbuscha). Canadian Journal of Fisheries and Aquatic Sciences 49:1305-1314.

Mobrand, L.E, J.A. Lichatowich, L.C. Lestelle, and T.S. Vogel. 1997. An approach to describing ecosystem performance "through the eyes of salmon". Canadian Journal of Fisheries and Aquatic Sciences 54: 2964-2973.

Nickelson, T.E., J.D. Rodgers, S.L. Johnson and M.F. Solazzi. 1992. Seasonal changes in habitat use by juvenile coho (Oncorhynchus kisutch) in Oregon coastal streams. Canadian Journal of Fisheries and Aquatic Sciences 49: 783-789.

Peterman, R.M. and J.L. Anderson. 1999. Decision analysis: a method for taking uncertainties into account in risk-based decision making. Human and Ecological Risk Assessment 5(2): 231-244.

Riley, S.C. and K.D. Fausch. 1995. Trout population response to habitat enhancement in size northern Colorado streams. Canadian Journal of Fisheries and Aquatic Sciences 52: 34-53.

Robb, C.A. and R.M. Peterman. 1998. Application of Bayesian decision analysis to the management of a sockeye salmon (Oncorhynchus nerka) fishery. Canadian Journal of Fisheries and Aquatic Sciences 55: 86-98.

Rodgers, J.D., M.F. Solazzi, S.L. Johnson, and M.A. Buckman. 1992. Comparison of three techniques to estimate juvenile coho salmon populations in small streams. North American Journal of Fisheries Management 12: 79-86.

Sainsbury, K.J. 1988. The ecological basis of multispecies fisheries and management of a demersal fishery in tropical Australia, p. 349-382 In J.A. Gulland (ed.). Fish population dynamics, 2nd edition. Wiley. New York.

Sandercock, F.K. 1991. Life history of coho salmon (Oncorhynchus kisutch) in Pacific Salmon Life Histories, C. Groot and L. Margolis eds. UBC Press: Vancouver.

Shardlow, T., R. Hilborn, and D. Lightly. 1987. Components analysis of instream 
escapement methods for Pacific salmon (Oncorhynchus spp.) Canadian Joumal of Fisheries and Aquatic Sciences 44: 1031-1037.

Sheng, Mel. 1992. Coho habitat restoration and development in the interior of BC in Proceedings of the Coho Salmon Workshop, p. 318-322.

Sheng, M.D., M. Foy, and A.Y. Fedorenko. 1990. Coho salmon enhancement in British Columbia using improved groundwater-fed side channels. Canadian Manuscript Report of Fisheries and Aquatic Sciences 2071.

Thedinga, J.F., M.L. Murphy, S.W. Johnson, J.M. Mitchel Lorenz, and K.V. Koskie. 1994. Determination of salmonid smolt yield with rotary-screw traps in the Situk River, Alaska, to predict effects of glacial flooding. North American Joumal of Fisheries Management 14: 837-851.

Walters, C.J. 1977. Management under uncertainty, p. 261-297. In D. Ellis (ed.). Pacific Salmon: Management For People.

Walters, C.J. 1986. Adaptive Management of Renewable Resources, MacMillan. New York. 374 pp.

Walters, C. 1992. Where have all the coho gone? in Proceedings of the Coho Salmon Workshop, p. 1-8.

Walters, C.J. and R. Green. 1997. Valuation of experimentai management options for ecological systems. Journal of Wildlife Management 61(4):987-1006.

Walters, C.J. and E.R. Keeley. 1994. The British Columbia watershed restoration program: summary of the experimental design, monitoring, and restoration techniques workshop. Watershed Restoration Management Report No. 1. Ministry of Environment, Land and Parks and Ministry of Forests. 


\section{Appendix - Components of Variance in Observed Effect Size}

This appendix provides details on the derivation of estimates of variance in the different components of effect size of enhancement. Estimates of measurement error variance were derived from a review of literature on the precision of different methods used to estimate fish abundance at various life-stages. As described below, these sources correspond with the "high-precision" and "low-precision" sampling methods described in Tables 7 and 8. Due to uncertainty to the wide variation in estimates of precision even for a given sampling method and species/life-stage of fish, I performed the analysis with "optimistic" and "pessimistic" estimates for measurement error variance. Variance estimates for process errors in effect size were derived from estimates of interannual variability in coho and chum survival rates reported in Bradford (1995) under two alternative assumptions. In the first case, I assumed that all of the variability in coho and chum survival rates was due to process error. However, some of the variability in survival rates reported in Bradford is likely due to measurement error, hence, I also derived estimates of process error variance after deducting "reasonable" estimates of measurement error variance. Below, I first describe the theory behind the components of variance of observed effect size. Then I apply the theory to data to estimate variance parameters used in the decision analysis.

\section{Theory of components of variance in effect size}

In my analysis, I define the observed effect size for any given system and year to be the difference in the natural logarithms of enhanced and wild survival rates for each particular life-stage and species. First, I will consider the observed effect size for the 
coho egg-to-fry survival rate, but the subsequent analyses for coho fry-to-smolt and chum egg-to-fry survival rates are analogous:

(A1) $\hat{\theta}_{i t}=\ln \left(\frac{F \hat{y} y}{E g g}\right)_{E, i t}-\ln \left(\frac{F \hat{y} y}{E g g}\right)_{W, i t}$

I define the following statistical model for the observed effect size in system $i$ and year $t$ :

(A2) $\hat{\theta}_{i t}=\mu+X_{i}+Y_{t}+e_{i t}+v_{i t}$

To derive estimates for the variances for the random terms in equation (A2), it is necessary to further break down variance into components of variation contained within observed survival rates for enhanced and wild fish. The following equations were used to represent the observed survival rates in enhanced and wild fish:

(A3) $\left(\frac{F \hat{y} y}{E g g}\right)_{E, i t}=\exp \left(\mu_{E}+X_{E, i}+Y_{E, s}+e_{E, i t}+v_{E, i t}\right)$
(A4) $\left(\frac{F \hat{y}}{E g g}\right)_{W, i t}=\exp \left(\mu_{W}+X_{W, i}+Y_{W, s}+e_{W, i t}+v_{W . i t}\right)$

where $\mu_{E}$ and $\mu_{W}$ are the mean logged survival rates for enhanced and wild fish, respectively. The $X$ terms are random system-specific effects that represent inherent differences in survival rates from system to system, the $Y$ terms are random year-specific effects that represent differences in survival rates from year to year that are shared across systems, the $e$ 's represent natural (i.e. process) variability in survival rates, and the $v$ 's represent measurement errors in survival rates. All of the random components in equations (A3) and (A4) were assumed to be normally distributed with mean of zero. Substituting (A3) and (A4) into equation (A1) and rearranging gives: 
(A5)

$$
\hat{\theta}_{i t}=\left(\mu_{E}-\mu_{W}\right)+\left(X_{E, i}-X_{W, i}\right)+\left(Y_{E, s}-Y_{W, d}\right)+\left(e_{E, i t}-e_{W, i t}\right)+\left(v_{E, i t}-v_{W, i t}\right)
$$

Hence, the variance of each random term in equation (A2) is equal to the variance of the difference of the corresponding random terms in equations (A3) and (A4). Data were not available to suggest what the variances of system-specific effects or year-specific effects might be. However, estimates of variance in measurement error and process error could be derived from reviews of the literature with the help of simplifying assumptions.

\section{Measurement error variance}

To derive estimates for variance in the measurement error for effect size, I assumed that measurement errors in abundance are log-normally distributed. Assuming log-normal measurement error implies that estimates of population abundance will tend to be skewed, which could arise if fish tend to aggregate and only a portion of a stream is sampled. For example, when spawners tend to aggregate, there is a high probability that a sampled area will contain a small number of fish and a low probability that the area will contain a large number of aggregated spawners, which could result in skewed estimates of population size for the entire stream (Irvine et al. 1994).

Under the assumption of log-normal errors in estimates of abundance, estimated abundance is related to true abundance by the following equation:

(A6) $\hat{N}=N \bullet \exp \left(v_{N}\right)$

where $\hat{N}$ is estimated abundance, $N$ is true abundance, and $v_{N}$ is the measurement error term with mean zero and variance $\sigma_{v, N}^{2}$. Following from this equation, estimated egg-tofry survival rates, for example, will be related to true egg-to-fry survival rates by the 
following equation:

(A7) $\left(\frac{F \hat{r y}}{E g g}\right)=\left(\frac{F r y}{E g g}\right) \bullet \exp \left(v_{f y y}-v_{e g g}\right)$

where $v_{f r y}$ and $v_{\text {egg }}$ are simply measurement errors in estimates of fry and egg abundance, respectively. Recall that I defined effect size as the difference in natural logarithms of enhanced and wild survival rates. Hence, the measurement error in effect size is:

(A8) Measurement error $=\left(v_{f y, E}-v_{\text {egg. E }}\right)-\left(v_{f f r . W}-v_{\text {egg.,W }}\right)$

where the $v$ 's are measurement errors terms in abundance estimates for each life-stage and habitat type. Assuming that measurement errors are uncorrelated, the variance of measurement error in effect size is simply the sum of the variance for the terms on the right hand side of equation (A8).

Precision of sampling methods to estimate abundance is most commonly reported in terms of coefficients of variation (CV). In order to obtain estimates for the variance in the error terms in abundance, I used the following relationship between the $\mathrm{CV}$ of abundance estimates and the variance of the ertor term in equation (A6) (Gelman et al. 1995):

(A9) $\sigma_{v, N}^{2}=\ln \left(C V^{2}+1\right)$

Once variances for measurement errors in relevant estimates of abundance are obtained, these can be summed to provide estimates for the measurement error variance in effect size.

\section{Process error}

The process error in observed effect size (equation A5) is given as: 
(A9) Process error $=\left(e_{E, i t}-e_{W, i t}\right)$

where $e_{E, i t}$ and $e_{W, i t}$ are process error terms in enhanced and wild survival rates, respectively. Hence, the variance of process error in effect size is:

(A10) $\sigma_{e}^{2}=\sigma_{E, e}^{2}+\sigma_{W, e}^{2}-2 \sigma_{E W, e}$

where the first two terms are the variances in process errors in natural logarithms of enhanced and wild survival rates and the third term is the covariance between process errors in logged enhanced and wild survival rates. Using the properties of the covariance of two random variables (Devore, 1991), equation (A10) can be rewritten in terms of the correlation between process errors $\left(\rho_{E w, c}\right)$ in equation (A9):

(AII) $\sigma_{e}^{2}=\sigma_{E, e}^{2}+\sigma_{W, e}^{2}-2 p_{E W, e} \bullet \sigma_{E, e} \bullet \sigma_{W, e} \cdot$

Below, I made certain assumptions regarding the relative magnitude of variances of process errors and the correlation of process errors; thus equation (All) can be solved strictly in terms of $\sigma_{w, e}^{2}$.

[ assumed that enhanced survival rates are less variable than wild survival rates and that there is a positive correlation between natural fluctuations in wild and enhanced survival rates within the same system and year. Enhanced egg-to-fry survival rates may exhibit less natural variability than wild survival rates, because side channels provide a more stable incubation environment (Bonnell, 1991). The same may also be true for fryto-smolt survival rates because side channels provide juvenile fish with protection from extreme weather conditions, such as low flows during summer and freshets during winter (Sheng et al., 1990). The positive correlation between process errors is justified on the assumption that causes of natural variation have the same directional effect on survival of 
incubating salmon and juveniles in a side channel and in the wild. However, as my previous assumption implies, the side channel environment provides fish using them with more of a buffer against such conditions. Specifically, I assumed that (a) the variance of the process error for enhanced fish is only half of that for wild fish and (b) the correlation of process errors between wild and enhanced fish is 50 percent (i.e. $\sigma_{E, e}^{2}=0.5 \bullet \sigma_{W, \varepsilon}^{2}$ and $\rho_{E W . \varepsilon}=0.5$ ). With these assumptions, the variance of process error in the observed effect size is:

(A10) $\sigma_{e}^{2}=0.793 \bullet \sigma_{W, e}^{2}$

\section{Data and Results}

\section{$\underline{\text { Measurement error }}$}

Coefficients of variation of abundance estimates using different sampling methods are summarized in Tables 7 and 8 by species, life-stage, and habitat type. Variances of measurement errors (Tables 9 and 10) in effect sizes were derived using these coefficients of variation as described above, except in the cases in which chum and coho egg-to-fry survival are estimated by hydraulic sampling, which does not provide a direct measure of abundance.

\section{Hydraulic sampling}

To quantify the efficacy of using hydraulic sampling as a surrogate measure of egg-to-fry survival rates, I used Bonnell's (199l) data on chum egg-to-fry survival rates in side channels, which were obtained by dividing fry outmigration (estimated with a 
counting fence) by potential egg deposition and corresponding estimates of egg-toembryo survival rates obtained through hydraulic sampling. I fit the following relationship to those data on fence-estimated egg-to-fry survival rates and embryo survival estimates:

(A11) $\ln \left(\frac{F y}{E g g}\right)=a+b \ln \left(\frac{E m b r y o}{E g g}\right)+v$

where $(F r y / E g g)$ is the egg-to-fry survival rate obtained from fence counts and (Embryo/Egg) is the egg-to-embryo survival rate. The resulting R-squared value from (A11) was 0.41 and the ertor variance was $0.447(\mathrm{~N}=27)$.

If I assume that the egg-to-fry survival rates obtained from fence counts were measured without error, then the variance of the measurement error from hydraulic sampling is simply equal to the variance of the error term in (A11). I made the further assumption that using hydraulic sampling to estimate egg-to-fry survival rates for coho would have the same measurement error as estimating egg-to-fry survival rates for chum. Hence, for the "low-precision" sampling methods, the variance of the measurement error term in equation (A2) for coho is: $\sigma_{v}^{2}=\sigma_{E, v}^{2}+\sigma_{W, v}^{2}=0.447+0.447=0.894$.

\section{Spawner abundance}

I assumed that spawner abundance in side channels can be estimated without error when a counting fence is used ("high-precision" monitoring program), i.e. $\mathrm{CV}=0$. In the "low-precision" monitoring program, it is assumed that spawners in the side channel are counted using visual surveys. Shardlow et al. (1987) report of $\mathrm{CV}$ of 0.1 for estimates of chum spawner abundance on a spawning riffle using a visual survey conducted on foot, 
hence I used this value for spawner abundance estimates in side channels.

I assumed that abundance of wild coho and chum spawners is estimated by using a counting fence to capture a portion of spawners to derive mark-recapture estimates of abundance in the "high-precision" monitoring program, whereas I have assumed that visual estimates are used in the "low-precision" sampling program. Irvine et al. (1999) reports coefficients of variation of approximately 0.1 for escapement estimates of coho spawner abundance to small streams in the Thompson River using mark-recapture methods. Hence, I used this value for the "optimistic" case. Cousens et al. (1982) state that CV's of 0.1 are considered good for estimates of spawner abundance with markrecapture methods, while CV's of 0.2 are considered average. Hence, in the "pessimistic" case, I used a CV of 0.2 .

\section{Chum fry and coho smolt abundance}

In both the "high-precision" and "low-precision" sampling programs, I assumed that chum salmon fry and coho salmon smolt abundances in the side channel would be estimated using a counting fence. As with spawner abundance, I assumed no measurement error in estimates of juvenile abundance in channels when using counting fences. Traps would be used to capture a portion of wild chum fry and coho smolts in the "high-precision" monitoring program. Estimates of catch efficiency would then be used to derive estimates for population abundance. Thedinga et al. (1994) obtained CV's for abundance estimates of sockeye, chinook, and coho salmon smolts ranging from 0.03 to 0.15 using rotary-screw traps in the Situk River, Alaska. I assumed a value of 0.1 in the "optimistic" case. However, when fish abundance and resulting catches are small, 
precision of catch efficiency and abundance estimates is reduced. Hence, in the "pessimistic" scenario, I assumed a CV of 0.2 , because catches of smolts ranged from 13,000 to 75,000 in Thedinga et al. (1994), which may be higher than what one might expect when using a trap in a small stream.

\section{Coho fry abundance}

In both the "high-precision" and "low-precision" monitoring programs, I assumed that coho fry abundance in the side channel would be estimated without error using a counting fence. Estimates of wild coho fry abundance would be estimated using markrecapture methods in the "high-precision" monitoring program, while in the "lowprecision" monitoring program, wild coho fry abundance would not be estimated directly. Instead, indirect estimates would be obtained by multiplying potential egg-deposition (determined through visual estimates of spawners) by estimated wild egg-to-fry survival rates obtained through hydraulic sampling.

Hankin and Reeves (1987) obtained a coefficient of variation of approximately 0.1 in estimates of coho salmon juveniles in Cummins Creek, Oregon using visual estimates of juvenile abundance obtained through diver counts. Hence, I used this value in the "optimistic" case for the "high-precision" monitoring program. Rodgers et al. (1992) obtained coefficients of variation close to 0.25 for estimates of population size using mark-recapture estimation in a number of pools in Beaver Creek, Oregon, which had been stocked with known numbers of coho juveniles. I used this value for the "pessimistic" case. 


\section{Process error}

Bradford (1995) reports variances of $0.178,0.514$, and 0.444 for the natural logarithms of coho egg-to-fry, coho egg-to-smolt, and chum egg-to-fry survival rates. Assuming that coho egg-to-fry and fry-to-smolt survival rates are independent, the variance in the coho fry-to-smolt survival rate is simply equal to variance in egg-to-smolt survival rate minus variance in the egg-to-fry survival rate. Hence, I used a value of $0.514-0.178=0.336$ for the variance in the $\log$ of coho fry-to-smolt survival rates. These values represent the variances for natural logarithms of wild survival rates.

Assuming that all of the variation in survival rates estimates from Bradford (1995) is due to natural (process) variation, these values for variance in natural logarithms of wild survival rates can be substituted into equation 10 to obtain estimates of process error variance in effect sizes. These translate into values of $0.141,0.266$, and 0.352 for process error variances for coho egg-to-fry, coho fry-to-smolt, and chum egg-to-fry life-stages, respectively.

However, some of the variation in survival sates reported by Bradford (1995) is likely due to measurement ertor, though the magnitude of measurement error is unknown. In most cases reviewed in Bradford (1995), traps were used to estimate fry and smolt abundance but sampling methods used to estimate spawners (and potential egg deposition) were not reported. I assumed that in these studies, coefficients of variation in abundance estimates for spawners, fry, and smolts for both coho and chum were equal to 0.2. Assuming that errors in abundance estimates are log-normally distributed (equation A6), CV's of 0.2 translate into variance estimates for the logged error term of $\ln \left(0.2^{2}-1\right)=$ 
0.039 (see equation A9). This implies a measurement error variance in logged survival rate estimates of $0.039 \times 2=0.078$. Hence, I deducted 0.078 from estimates of variance reported in Bradford (1995), yielding estimates of process error variance in coho egg-tofry, coho egg-to-smolt, and chum egg-to-fry survival rates of $0.100,0.436$, and 0.366 , respectively. As before, I subtracted the variance in logged coho egg-to-fry survival rates from the variance in logged coho egg-to-smolt survival rates to obtain an estimate of the variance in logged coho fry-to-smolt survival, giving 0.336 . Using equation $A 9$, these values for variance in natural logarithms of wild survival rates translate into estimates of process error variance in effect sizes of $0.079,0.266$, and 0.290 for coho egg-to-fry, coho fry-to-smolt, and chum egg-to-fry life-stages, respectively. 